\title{
Applying Data Preparation Methods to Optimize Preterm Birth Prediction
}

\author{
by
}

\author{
Alana Esty, B. Eng.
}

A thesis submitted to the Faculty of Graduate and Postdoctoral Affairs in partial fulfillment of the requirements for the degree of Master of Applied Science in Biomedical Engineering

Ottawa - Carleton Institute for Biomedical Engineering (OCIBME)

\author{
Carleton University \\ Ottawa, Ontario
}

July 2018

(C) 2018

Alana Esty 


\section{Abstract}

The purpose of this work was to develop an accurate prediction model which can process information contained in antenatal databases to determine whether a baby will be born prematurely. The focus was on improved data preprocessing to add to methods developed by previous students in the Carleton MIRG (Medical Information technology Research Group) lab.

The machine learning classifiers used included Decision Tree (DT) classifiers (for feature reduction) and the Artificial Neural Network (ANN) classifier (for model evaluation). Missing values and class imbalance was dealt with by applying software packages in the $\mathrm{R}$ statistical programming language.

This research has shown a marked improvement in the accuracy of predicting preterm births. The final sensitivity and specificity results for the BORN (Better Outcomes Registry and Network) database were: Parous $89.2 \%$, and $67.8 \%$, Nulliparous $89.0 \%$ and $71.5 \%$, and for PRAMS (Pregnancy Risk Assessment Monitoring System) database: Parous 84.1\% and $71.4 \%$, Nulliparous $83.8 \%$ and $76.0 \%$. These improved results are promising. An accurate predictive tool will allow caregivers to implement preventative treatment strategies or to ensure delivery occurs in a tertiary health care Centre. 


\section{Acknowledgements}

I would like to thank my thesis supervisor, Dr. Monique Frize, for her support, advice and guidance throughout my degree. Thank you for the opportunity to be exposed to and work on a variety of enriching projects and workshops.

Thank you to my co-supervisor, Dr. Jeff Gilchrist who provided exceptional feedback and mentorship throughout my degree.

Thank you to my co-supervisor, Dr. Erika Bariciak at the Children's Hospital of Eastern Ontario who was always available for questions and provided detailed and relevant feedback and support.

I am also thankful for my parents who have consistently supported me through both the highs and lows of my graduate degree and have always encouraged me to strive for the best I can.

I would also like to thank Guy Kouamou Ntonfo, Carole Love, everyone at the Carleton GSA and of course, Dawn Patrice Collins Gregory, I am very grateful for their support and encouragement. 


\section{Table of Contents}

Abstract ............................................................................................................................. ii

Acknowledgements ....................................................................................................................ii

Table of Contents..................................................................................................................... iv

List of Tables ................................................................................................................. vii

List of Figures ……………………………………………………………… ix

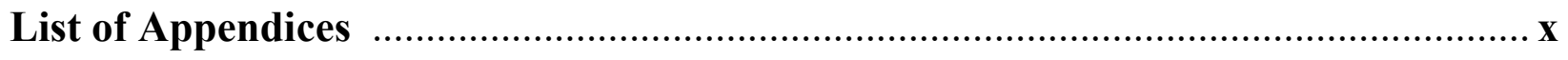

List of Acronyms …………………………………………………………... $\mathbf{x i}$

1 Chapter: Introduction ............................................................................................... 1

1.1. Motivation .....................................................................................................................

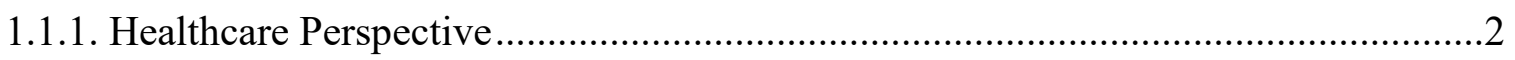

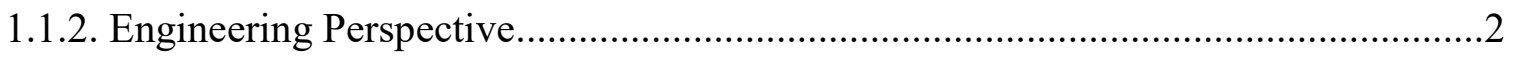

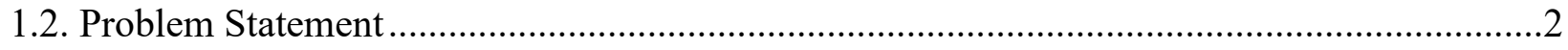

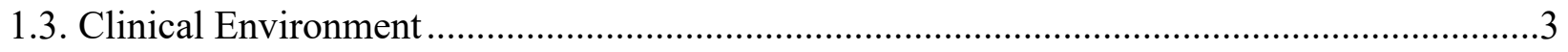

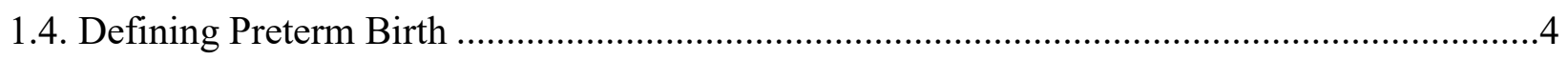

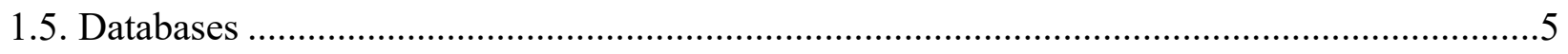

1.5.1. Segmenting the databases .............................................................................6

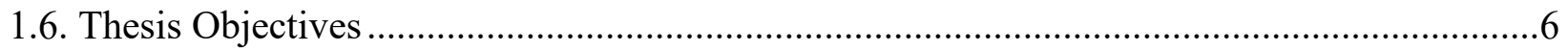

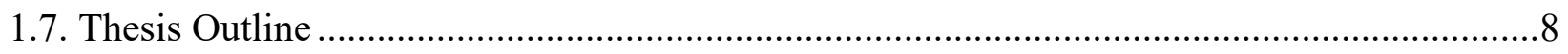

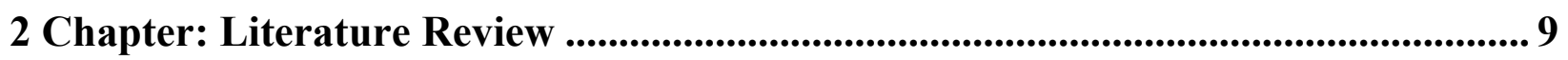

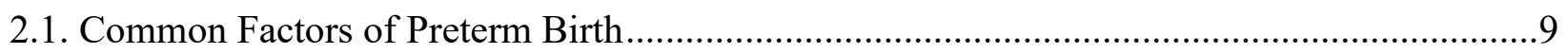

2.1.1. Social Stress and Race ………………………..................................................

2.1.2. Infection and Inflammation............................................................................

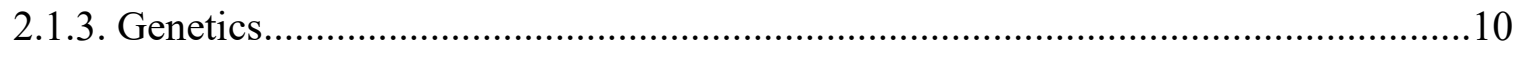

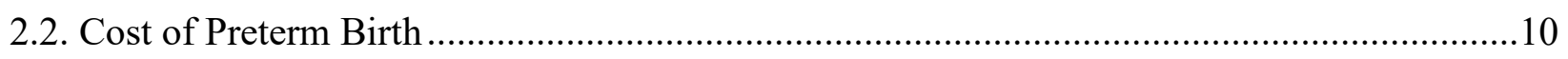

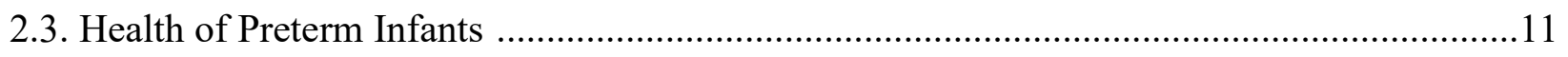

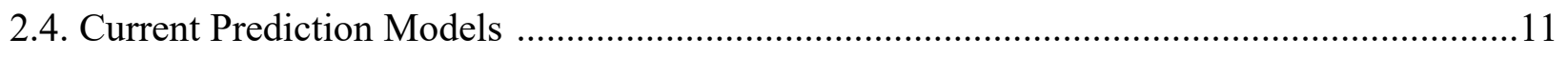

2.4.1. Cervical Length ...........................................................................................11

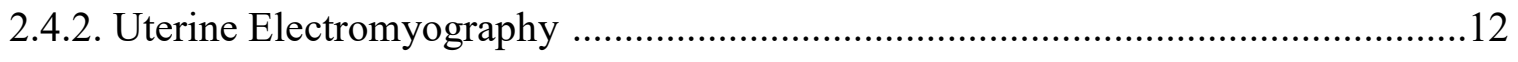

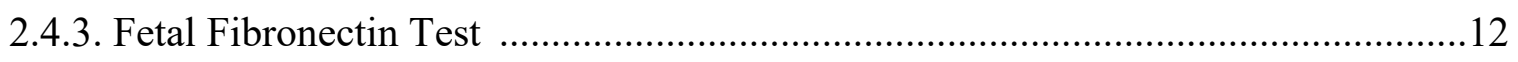

2.4.4. Physician-Parent Decision Support (PPADS) .....................................................13 
2.4.5. Ontario Perinatal Record .....................................................................................

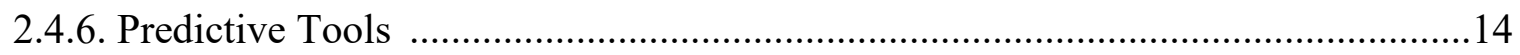

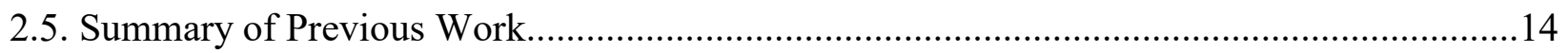

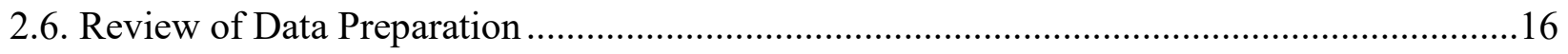

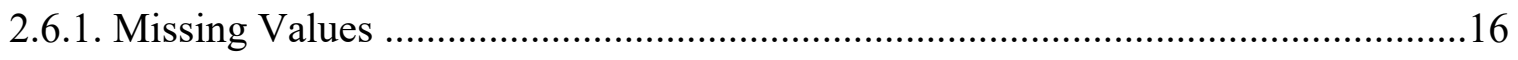

2.6.2. Discussion of Alternative Imputation Methods ......................................................18

2.6.3. Simple Imputation Methods ………………………............................................18

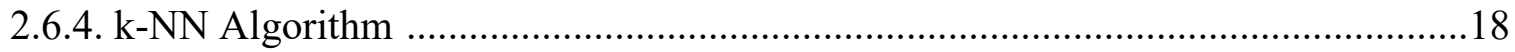

2.6.5. mice Algorithm ..........................................................................................19

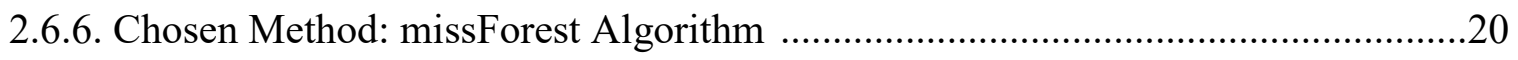

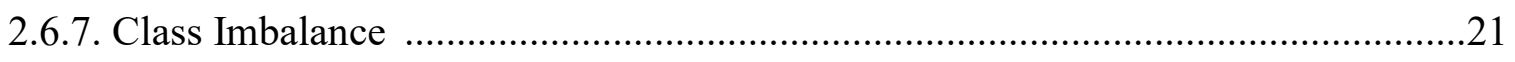

2.6.8. Discussion of Alternative Class Imbalance Methods ............................................21

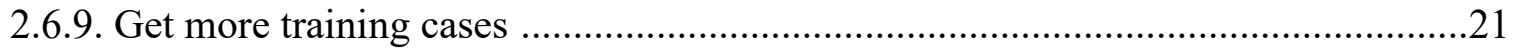

2.6.10. Oversampling the minority class ……………..................................................22

2.6.11. Chosen Method: Undersampling the majority class ...........................................22

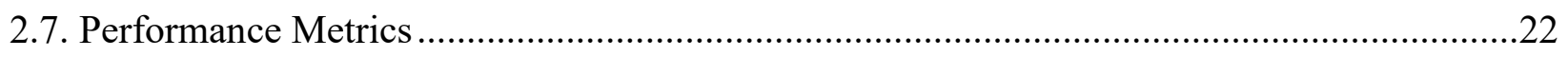

2.7.1. Confusion Matrix (Contingency Table) ……………………………………......23

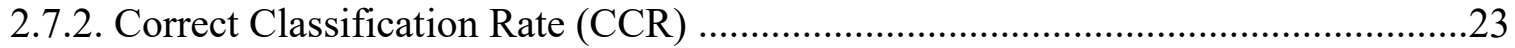

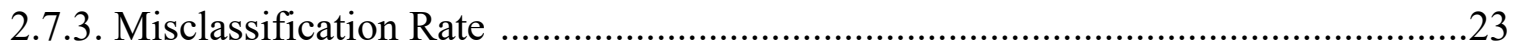

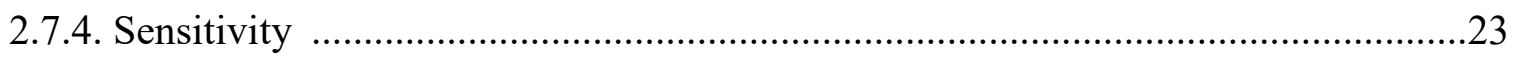

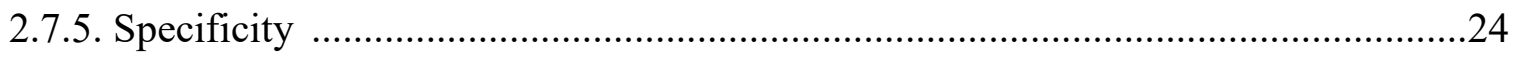

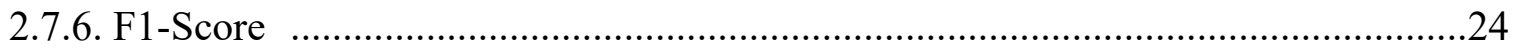

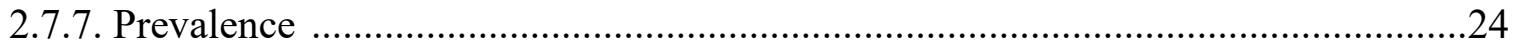

2.7.8. Positive Predictive Value \& Negative Predictive Value...........................................25

2.7.9. Receiver Operating Characteristic (ROC) Curve ....................................................25

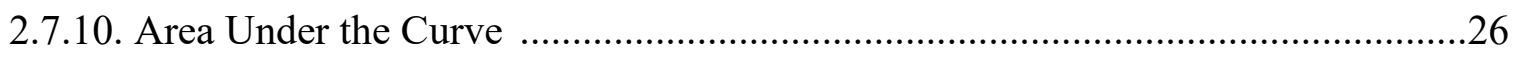

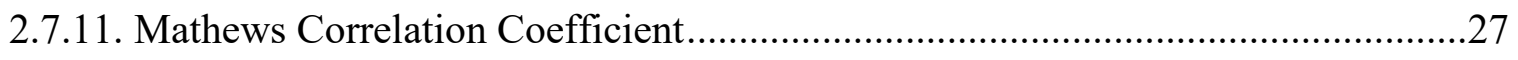

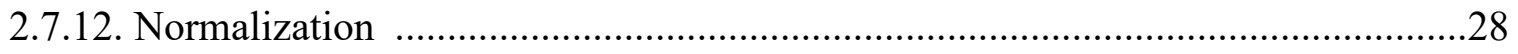

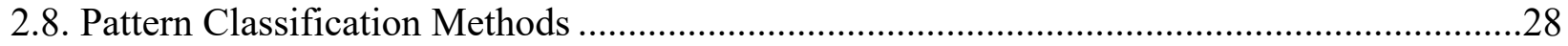

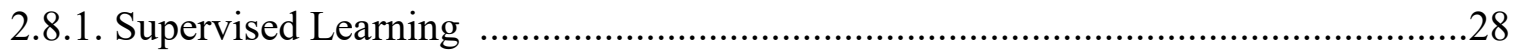

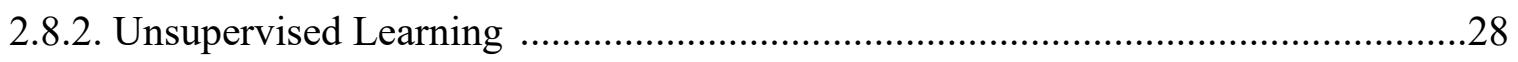

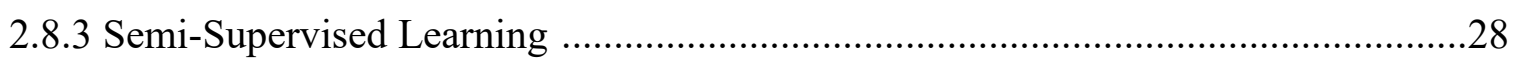




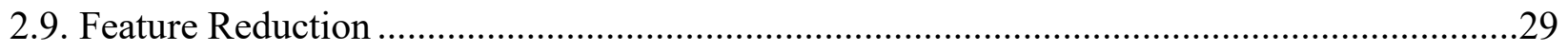

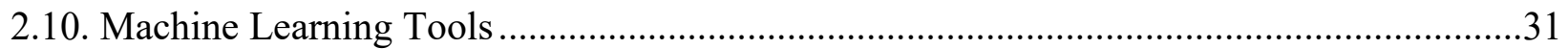

2.10.1. Decision Tree Classifier...................................................................................

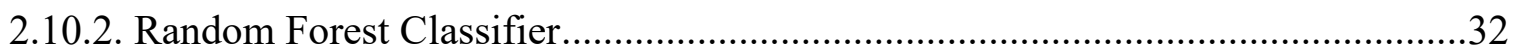

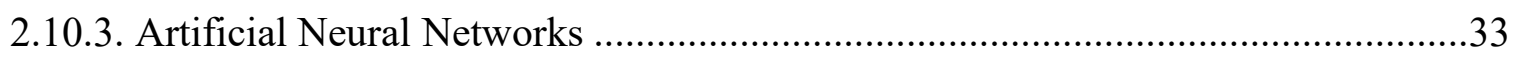

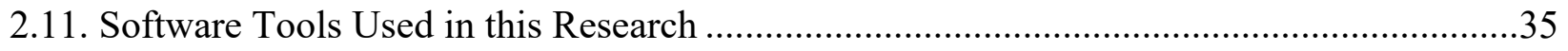

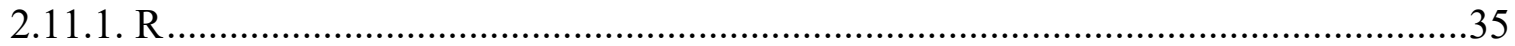

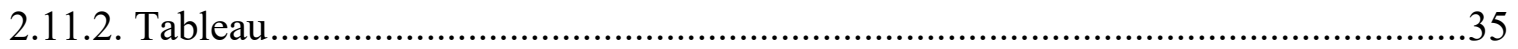

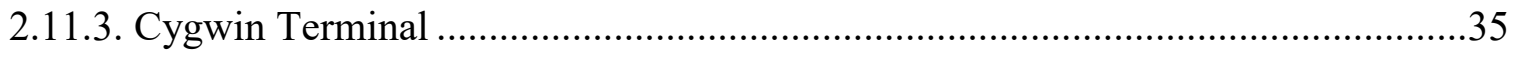

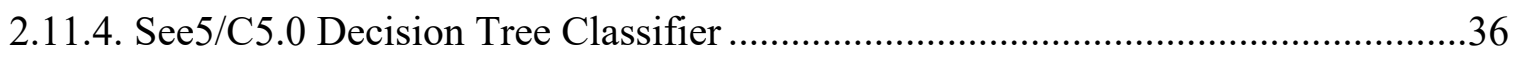

2.11.5. Fast Artificial Neural Network Library ................................................................36

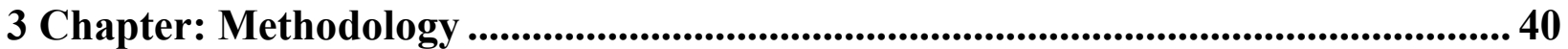

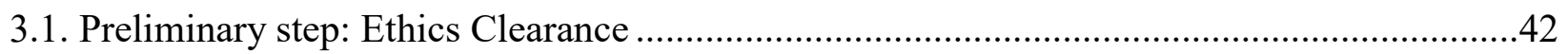

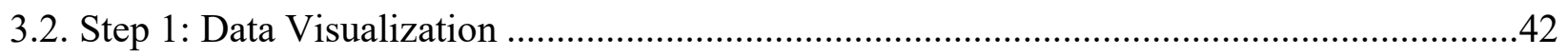

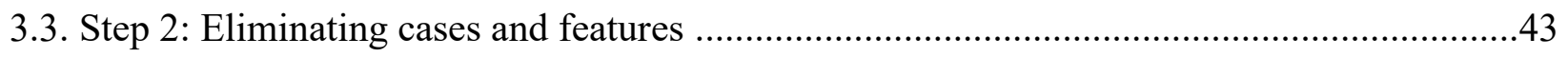

3.4. Step 3: Choosing features with greater than $50 \%$ importance using the

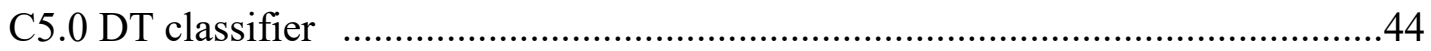

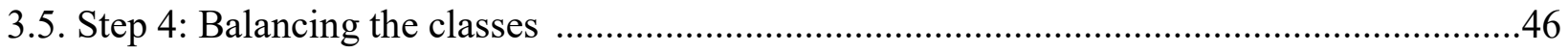

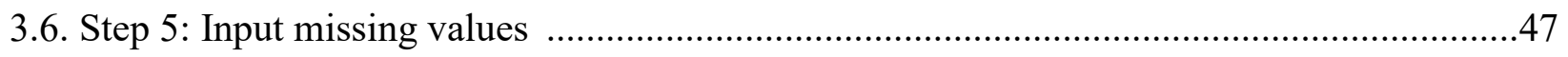

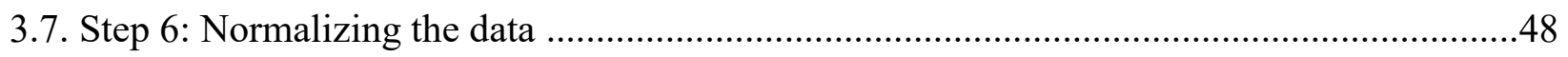

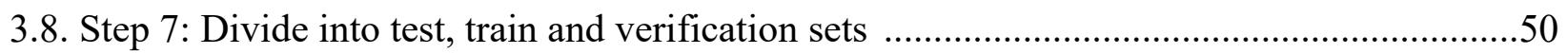

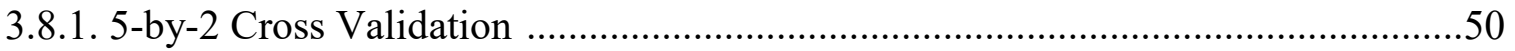

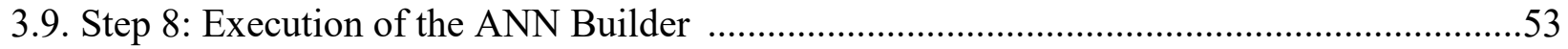

4 Chapter: Results and Discussion........................................................................................5 57

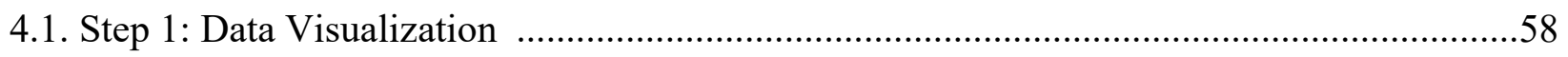

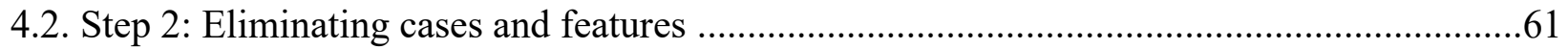

4.3. Step 3: Choosing features with greater than $50 \%$ importance using the

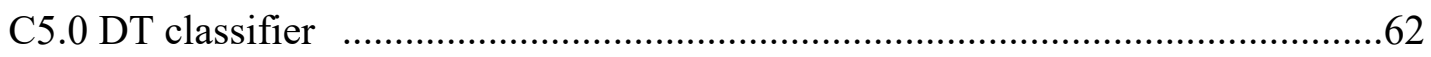

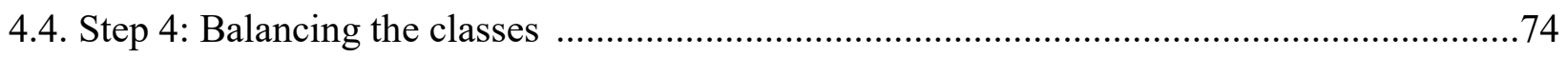

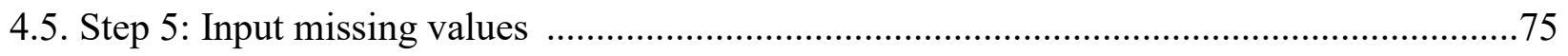

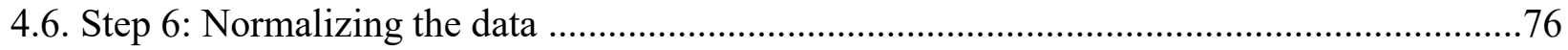

4.7. Step 7: Divide into test, train and verification sets ............................................................ 


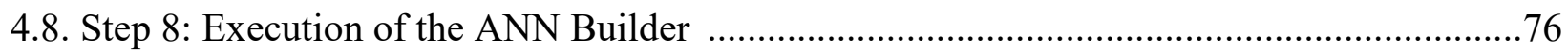

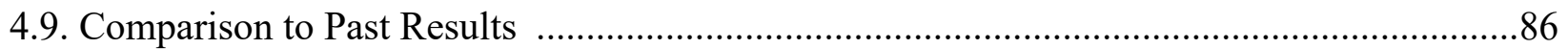

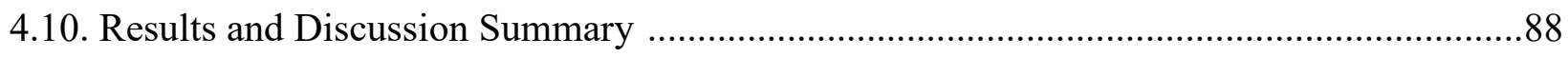

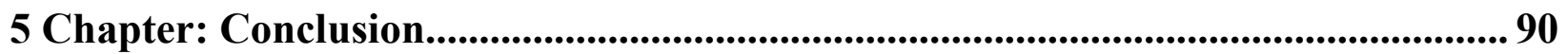

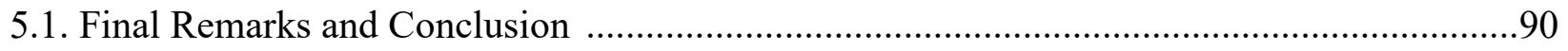

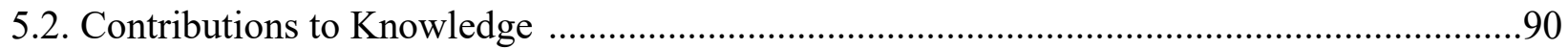

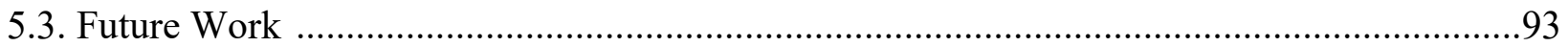

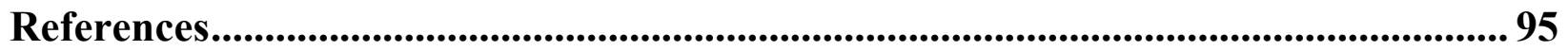

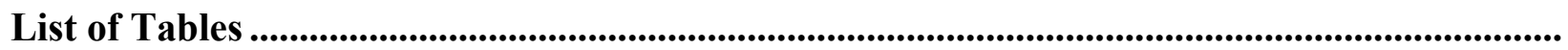

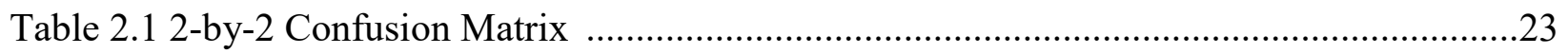

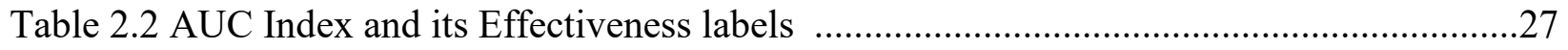

Table 3.1 Methodology for the development and evaluation of the predictive tool ....................41

Table 3.2 Description of parameters for package in $\mathrm{R}$ (ubBalance) .............................................46

Table 3.3 Description of parameters for package in $\mathrm{R}$ (missForest) ............................................48

Table 3.4 Division of train, test and verification sets ............................................................50

Table 4.1 Results for the development and evaluation of the predictive tool ...............................57

Table 4.2 Number of features prior to and after feature and case elimination ............................61

Table 4.3 Comparison of the two methodologies ................................................................63

Table 4.4 Increased feature size to include $\geq 30 \%$ feature importance (BORN) ………...............64

Table 4.5 Reduced feature size to include $\geq 65 \%$ feature importance (BORN) ...........................65

Table 4.6 Increased feature size to include $\geq 30 \%$ feature importance (PRAMS) .........................65

Table 4.7 Reduced feature size to include $\geq 65 \%$ feature importance (PRAMS) ..........................65

Table 4.8 Feature reduction result after applying the C5.0 DT classifier to the BORN and

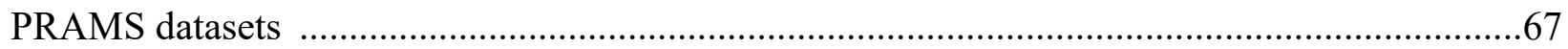

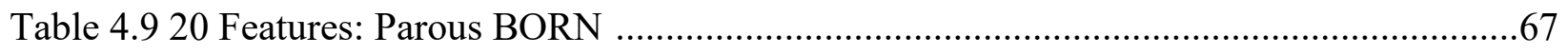

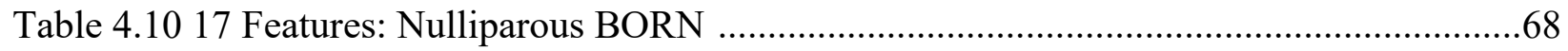

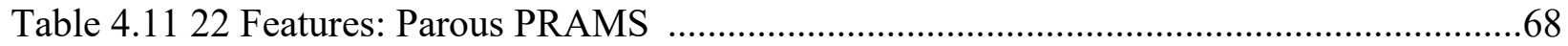

Table 4.12 19 Features: Nulliparous PRAMS …………..................................................69

Table 4.13 Similar features chosen in current and earlier research work: Parous_BORN ...........72

Table 4.14 Similar features chosen in current and earlier research work: Nulliparous_BORN ...72 
Table 4.15 Similar features chosen in current work and earlier research work: Parous_PRAMS

Table 4.16 Similar feature chosen in current work and earlier research work:

Nulliparous_PRAMS .74

Table 4.17 Case reduction results after applying package in $\mathrm{R}$ (ubBalance) to the BORN and PRAMS datasets .75

Table 4.18 OOB error estimate for Nulliparous_PRAMS dataset .75

Table 4.19 Performance Metrics for the PRAMS_Parous classifier .77

Table 4.20 Confusion Matrix: Parous_BORN Verification Results at 7.9\% Prevalence Unseen

Data

Table 4.21 Performance Metrics Parous_BORN Verification results at 7.9\% Prevalence Unseen Data .80

Table 4.22 Confusion Matrix: Nulliparous_BORN Verification Results at 7.9\% Prevalence Unseen Data

Table 4.23 Performance Metrics: Nulliparous_BORN Results at 7.9\% Prevalence Unseen Data

Table 4.24 Confusion Matrix: Parous_PRAMS Verification Results at 7.9\% Prevalence Unseen

Data

Table 4.25 Performance Metrics: Parous_PRAMS Verification Results at 7.9\% Prevalence Unseen Data

Table 4.26 Confusion Matrix: Nulliparous_PRAMS Verification Results at 7.9\% Prevalence Unseen Data

Table 4.27 Performance Metrics: Nulliparous_PRAMS Verification Results at 7.9\% Prevalence Unseen Data .83

Table 4.28 Display of the Artificial Neural Network results for BORN and PRAMS datasets ...86

Table 4.29 Display of the Artificial Neural Network results for past results (2015).................87

Table 4.30 Display of the Artificial Neural Network results for past results (2009)..................87

Table 4.31 Display of the Artificial Neural Network results for past results (2007). .87 


\section{List of Figures.}

Figure 2.1 Regression methods in the mice algorithm to impute missing values .19

Figure 2.2 ROC curve and the different points of significance .26

Figure 2.3 Depiction of the Decision Tree Classifier Framework .31

Figure 2.4 Depiction of the Random Forest Classifier Framework .33

Figure 2.5 Depiction of the Activation Function and Artificial Neural Network Framework .....34

Figure 3.1 Schematic representation of the methodology used for the preterm birth classification tool. 40

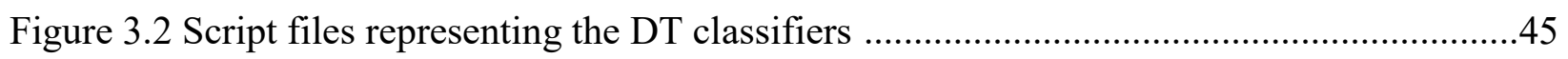

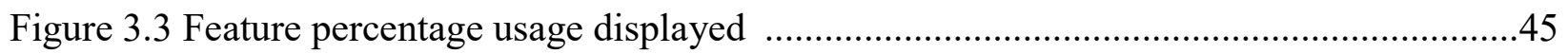

Figure 3.4 5-by-2 Cross Validation to create train, test and verification sets ...............................52

Figure 3.5 Parameters for the BORN_Nulliparous dataset ........................................................54

Figure 4.1 Bar Chart in Tableau comparing Parous_PRAMS features ......................................59

Figure 4.2 Missingness Map for the BORN_Nulliparous features .............................................60

Figure 4.3 Missingness Map for the PRAMS_Nulliparous features ..........................................61

Figure 4.4 List of abbreviations used for highly ranked features which occurred in both the BORN and PRAMS data sets, used in this study to assess risk of preterm birth

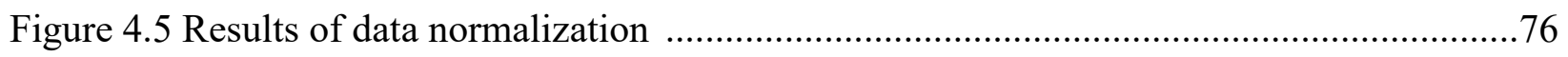

Figure 4.6 Results of 5-by-2 Cross Validation (test set) ........................................................76

Figure 4.7 Division of the BORN and PRAMS dataset: training, testing, verification and

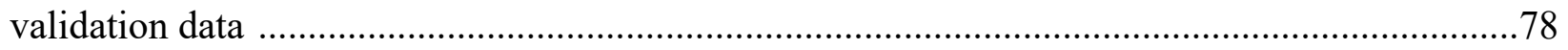

Figure 4.8 ROC Curve Performance for BORN_Parous Dataset ..............................................84

Figure 4.9 ROC Curve Performance for BORN_Nulliparous Dataset ..........................................85

Figure 4.10 ROC Curve Performance for PRAMS_Parous Dataset ............................................85

Figure 4.11 ROC Curve Performance for PRAMS_Nulliparous Dataset .......................................86 


\section{List of Appendices}

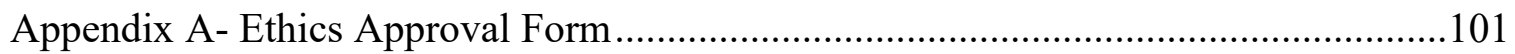

Appendix B- Description of BORN and PRAMS Features..........................................102

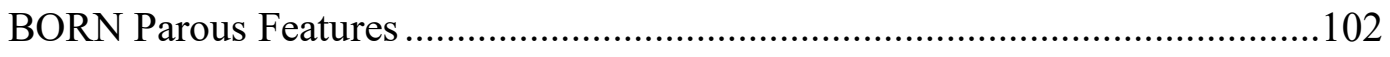

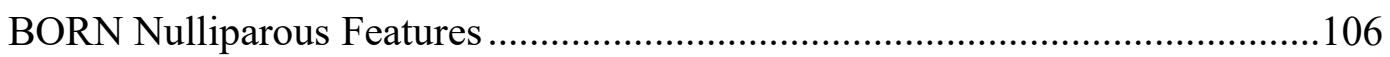

PRAMS Parous Features............................................................................ 110

PRAMS Nulliparous Features....................................................................112

Appendix C- Description of ANN Final Network Parameters ...........................................114

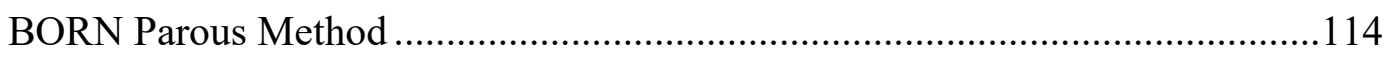

BORN Nulliparous Method .........................................................................117

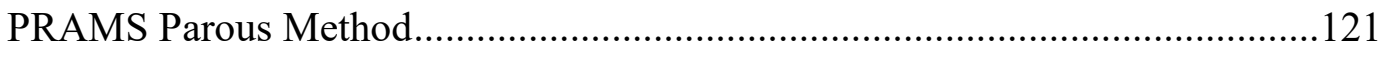

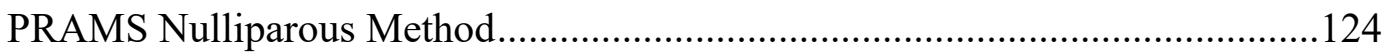




\section{List of Acronyms}

\begin{tabular}{|c|c|}
\hline $\mathrm{ACC}$ & Accuracy \\
\hline $\mathbf{A N N}$ & Artificial Neural Network \\
\hline AOM & Association of Ontario Midwives \\
\hline APGAR & $\begin{array}{l}\text { Activity, Pulse, Grimace, } \\
\text { Appearance and Respiration }\end{array}$ \\
\hline $\mathbf{A U C}$ & Area Under Curve \\
\hline BASH & Bourne-Again Shell \\
\hline BORN & Better Outcomes Registry Network \\
\hline BP & Blood Pressure \\
\hline CBR & Case-Based Reasoning \\
\hline CCR & Correct Classification Rate \\
\hline CDC & $\begin{array}{l}\text { Centers for Disease Control and } \\
\text { Prevention }\end{array}$ \\
\hline CDR & Clinical Data Repository \\
\hline СНEO & $\begin{array}{l}\text { Children's Hospital of Eastern } \\
\text { Ontario }\end{array}$ \\
\hline $\mathbf{C L}$ & Cervical Length \\
\hline CSV & Comma Separated Value \\
\hline DT & Decision Tree \\
\hline FANN & Fast Artificial Neural Network \\
\hline fFN & Fetal Fibronectin \\
\hline FN & False Negative \\
\hline FP & False Positive \\
\hline FS & Feature Selection \\
\hline NICU & Neonatal Intensive Care Unit \\
\hline k-NN & k-Nearest Neighbour \\
\hline LBW & Low Birth Weight \\
\hline MAR & Missing at Random \\
\hline MCAR & Missing Completely at Random \\
\hline MCC & Matthews Correlation Coefficient \\
\hline MICE & $\begin{array}{l}\text { Multivariate Imputation via Chained } \\
\text { Equations }\end{array}$ \\
\hline MIRG & $\begin{array}{l}\text { Medical Information technologies } \\
\text { Research Group }\end{array}$ \\
\hline MLP & Multilayer Perceptron \\
\hline MSE & Mean Squared Error \\
\hline NICU & Neonatal Intensive Care Unit \\
\hline NMAR & Not Missing at Random \\
\hline NPV & Negative Predictive Value \\
\hline OPR & Ontario Perinatal Record \\
\hline OMA & Ontario Medical Association \\
\hline PHIPA & $\begin{array}{l}\text { Personal Health Information } \\
\text { Protection Act }\end{array}$ \\
\hline РСМСН & $\begin{array}{l}\text { Provincial Council for Maternal } \\
\text { Child Health }\end{array}$ \\
\hline
\end{tabular}




\begin{tabular}{|l|l|}
\hline PBNN & Pruning Based Neural Network \\
\hline PPADS & Physician-PArent Decision Support \\
\hline PPV & Positive Predictive Value \\
\hline PRAMS & $\begin{array}{l}\text { Pregnancy Risk Assessment } \\
\text { Monitoring System }\end{array}$ \\
\hline PTB & Preterm Birth \\
\hline RFW & Research Framework \\
\hline ROC & Receiver Operating Curve \\
\hline SQL & Structured Query Language \\
\hline TN & True Negative \\
\hline TP & True Positive \\
\hline EMG & Uterine Electromyography \\
\hline
\end{tabular}




\section{Chapter: Introduction}

The purpose of this introductory chapter is to provide a framework for this thesis, including the motivation for the research from both a healthcare and engineering perspective. In addition, an overview of the problem statement, a description of the clinical environment, preterm birth, the databases used, and the thesis objectives and outline are addressed.

\subsection{Motivation}

\subsubsection{Healthcare Perspective}

In the current healthcare environment data is constantly being collected by clinical and hospital equipment. The ability to access massive amounts of healthcare data is a gold mine for predicting future health outcomes [1]. Large companies such as Google, GE Health, and IBM have recognized the potential of these massive datasets and have developed algorithms for recognizing patterns in health data [2]. For instance, Google has developed machine learning algorithms to quickly identify health conditions [3].

This work analyzes two large clinical datasets containing antenatal health information: the Better Outcomes Registry and Network (BORN) Database [4] and the Pregnancy Risk Assessment Monitoring System (PRAMS) Database [5]. Premature birth can have critical long-term effects on the patient, the family and on the clinical environment. From a healthcare perspective, there can be a huge benefit in being able to flag women who might be at risk for preterm birth; this enables the health care team to apply preventative care and to decide how best to manage the delivery. Currently methods used by healthcare teams to try to predict preterm birth are invasive, not very accurate or reliable, and are only used once the patients presents with symptoms of potential preterm [6]. 


\subsubsection{Engineering Perspective}

The use of classifiers within the healthcare field is rapidly increasing. The role of software tools when analyzing "Big Data" is that these tools have capabilities to deal with massive amounts of data and can rapidly observe inherent patterns and correlations in clinical data [1]. This can ultimately aid clinicians during prevention, diagnosis and post-diagnosis stages to improve the care provided to patients. "Big Data" can encompass many different definitions, but in the case of this research work, it consists of large databases which contain a variety of patient data and information.

The machine learning techniques used in this thesis work combine both Decision Tree (DT) and Artificial Neural Network (ANN) classifiers to classify neonatal outcomes. The DT classifier implemented the C5.0 RuleQuest Research software [6]. The ANN classifier implemented the Fast-Artificial Neural Network (FANN) library [7]. From an engineering perspective, increasing the accuracy of classifiers to identify health outcomes can assist physicians in make a diagnosis, understanding prognosis, and developing tailored treatment plans.

\subsection{Problem Statement}

In the past, clinicians would make predictions of a patient's future outcomes based on that individual's medical history. The benefit of using machine learning techniques such as an integrated DT-ANN model, is that software tools can quickly draw upon a multitude of clinical features, resulting, ideally, in a more accurate prediction of outcome for the individual.

Data preprocessing is arguably one of the most important steps in the data mining process [8], [9], [10]. Data preprocessing contains many steps: data cleaning, feature selection, normalization 
and transformation of the data. Without this data preprocessing step, model evaluation can result in misleading and inaccurate results [10]. The two datasets analyzed for this research work contain raw, noisy, real-life data which needs to be preprocessed before entering the data into the ANN model.

This thesis represents continuation of work done by previous MIRG students including: Catley, Yu and Ong. Catley developed an early prediction model which used a combination of Multilayer Perceptron Artificial Neural Networks and a decision tree voting algorithm [11]. This hybrid machine learning classifier was then further developed by $\mathrm{Yu}$, who used the decision tree classifier to eliminate variables and then applied an artificial neural network with weightelimination, with improved sensitivity and specificity results [12]. Finally, Ong's work introduced a new neural network classifier using the Fast-Artificial Neural Network Library [13]. Compared to past research, which focused on the machine learning models, the primary focus of this work is on data preparation to improve the sensitivity metric. The emphasis is on sensitivity, as this performance metric describes the probability of the classifier correctly predicting preterm cases. A prediction model with a high sensitivity will also help to ensure positive cases are not missed. This is important, since the eventual integration of the classifier into a clinical setting will necessitate identification of the risk of preterm birth as early as possible in the pregnancy while not missing any positive cases.

\subsection{Clinical Environment}

Obstetrics is the area of medicine focused on childbirth and maternal health during childbirth. Preventing and predicting preterm birth is an important area in the field of obstetrics, since preterm birth is associated with decreased infant survival, increased risk for short term and longterm health complications, and an increased use of health care technology and expenses [14]. 
Tocolytic drugs are medications used to delay the onset of labour [14]. Research shows that there is no evidence that these drugs improve neonatal outcomes and can result in adverse effects for both the mother and baby [14]. Frequently, these drugs are used as a last resort before a preterm birth occurs. The focus of this research work is on predicting preterm birth, because with accurate, non-invasive prediction methods, physicians can apply antenatal interventions as early as possible and potentially improve birth outcome for infants.

\subsection{Defining Preterm Birth}

Preterm birth is defined as birth which takes place before 37 weeks of gestation [15]. In Ontario, the preterm birth rate is $7.9 \%$ [16]. In the US, the frequency of preterm birth is around $8-12 \%$ and in other developed nations in Europe the rate is around 5-9\% [17]. Often there is no definite identified cause of preterm birth; however, there are several socioeconomic, physiological and environmental factors which can contribute to the risk of a preterm birth [17]. Some of these factors include smoking, having previous children who were premature, and bacterial vaginosis [17]. In addition, the risk of infant mortality with a premature birth is generally quite high [18]. These infants at birth are still in the early stages of development and this can leave them more susceptible to illness and disease. For instance, premature infants often require mechanical ventilation, as their lungs have not fully developed. Many of these infants experience several chronic illnesses such as chronic lung disease and respiratory distress syndrome [19].These highrisk situations can be damaging for the long-term health of the infant and can result in short and long-term costs for hospitals.

The fetal fibronectin test is considered the current gold standard for predicting preterm birth, specifically for women with a history of preterm birth; however, the test is expensive and 
invasive [20], [21], [22]. In addition, the sensitivity of the test varies depending on the gestational week and the test can only be used once the patient presents with symptoms which are indicative of potential impending preterm birth [23]. Therefore, a less expensive method which can either meet or exceed the accuracy and timing of the current standard is desired.

\subsection{Databases}

The PRAMS database contains over 100,000 cases with over 300 general clinical features of state-specific population-based maternal and infant data [5]. This database was first developed in 1987, and although this questionnaire has been updated throughout the years, no major revisions have occurred since Phase 4 (2000-2003). In order to compare these results to those obtained in past thesis work, [24], the same database was used: Phase 6 (2009-2011). The PRAMS database covers around $83 \%$ of all U.S. births. This database collects standardized data in survey form from volunteers across 47 states. PRAMS is administered by the Centers for Disease Control and Prevention (CDC), Division of Reproductive Health, National Center for Chronic Disease Prevention and Health Promotion. It is mainly focused on data before, during, and after pregnancy, and its purpose is to collect data to identify groups who might be at risk for high-risk pregnancies and to prevent these occurrences in the future [5]. Around $20 \%$ of the dataset analyzed in this research contained preterm cases.

The BORN database contains over 600,000 patient cases with over 200 general clinical features of Ontario maternal and newborn data [4]. The BORN database is a prescribed registry which has the authority to automatically collect and track health data under the Personal Health Information Protection Act [25]. The BORN database is funded by the Ontario Ministry of Health and Long-Term Care and is administered by the Children's Hospital of Eastern Ontario 
(CHEO). Some of the areas of focus of the BORN database include: maternal newborn outcomes / midwifery, congenital anomalies surveillance, newborn screening, and prenatal screening [26]. This database focuses on cases solely from Ontario with data on pregnancy, birth, and childhood factors [4]. Around $8 \%$ of the dataset analyzed in this research contained preterm cases.

\subsubsection{Segmenting the databases}

The PRAMS and BORN database were further divided between Parous and Nulliparous cases. Parous women are women who have had previous births, whereas Nulliparous women are women who have not given birth previously. Therefore, specific features will only be applicable to Parous women (i.e. previous premature birth) and thus, will affect the performance metrics of the predictive tool. Features related to Parous and Nulliparous cases were selected with consultation from our clinical partner. This is important since certain parous features, such as previous premature birth, are known to be highly predictive of future preterm birth [17]. Although it is helpful to see how the predictive model performs for both of these case types, this predictive model should be applicable to the general population and be inclusive of all women, including those who have no prior history of preterm birth. Therefore, four datasets were modelled throughout this research: BORN_Parous, BORN_Nulliparous, PRAMS_Parous and PRAMS_Nulliparous, where the nulliparous group included both.

\subsection{Thesis Objectives}

The overall goal of this thesis was to develop a predictive tool which has improved sensitivity results when compared to past work done by our research group. To accurately make this comparison, the same methodology steps will be followed from Ong's work [24], except for the data preparation stage. The final goal is to be able to apply this tool prospectively at obstetrical clinics that log patient data electronically to help clinicians and provide information for families. 
To fulfill this goal, three objectives must first be addressed. The first objective was to evaluate the processed data for feature reduction. There were a multitude of features in both the BORN and PRAMS database; many of these were not related to predicting preterm birth. The C5.0 Decision Tree classifier was applied to create a subset of features most important for predicting preterm birth. Utilizing this subset of features enhances the accuracy of the Artificial Neural Network during training and testing.

The second objective of this thesis is to apply data mining techniques to the BORN and PRAMS databases, with a focus on data preparation. Addressing the presence of missing values and class imbalance were the two main areas of focus in the data preparation stage. The hypothesis was that the greatest improvement in sensitivity results would be achieved by focusing on these two areas.

The third objective was to evaluate the above hypothesis; by comparing the sensitivity metrics obtained in this work with those obtained from past research (Ong [24], Yu [27] and Catley [11]). The same machine learning tools were used (DT and ANN) in past work performed [24] and the results obtained were compared to the current prediction performance, when applying new data preparation methods. In addition, the 5-by-2 cross validation technique introduced in past research [24] was applied to reduce bias and overfitting of the Artificial Neural Network Classifier. This comparison was done to observe the differences in classification results, when there is a focus on improving data quality, prior to training and testing the predictive model. 
The final results should provide an assessment of the level of improvement provided by the new methodology; this approach could be followed when implementing a predictive tool at clinics collecting prenatal data, to ensure high accuracy of predicting preterm outcomes.

\subsection{Thesis Outline}

The outline of this thesis is as follows:

Chapter 1 outlines the motivation for this work, gives a general overview of the problem and a description on how this research work contributes to improving past research results.

Chapter 2 provides a background and detailed literature review of preterm birth. This chapter also provides a summary of past work done by researchers at the MIRG group and on data preparation methods; this section also explains, in depth, the performance metrics addressed in this research work. In addition, the machine learning classifiers and software tools used to evaluate the datasets analyzed in this work are addressed.

Chapter 3 describes the methodology of the research work: it focuses on the software tools and models used to analyze and test the clinical datasets.

Chapter 4 presents the results of the data preparation steps, model evaluation and contains a discussion on the performance metrics achieved in predicting outcomes for preterm birth, compared with previous results of other models.

Chapter 5 summarizes the final models and presents concluding remarks and the thesis contribution. In addition, this section provides suggestions for future work. 


\section{Chapter: Literature Review}

This chapter encompasses a review of the literature based on risk factors associated with preterm birth. It includes a review of past work done by students within the MIRG lab, data preparation methods and current prediction models. This chapter describes pertinent performance metrics that will appear in later chapters and summarizes the machine learning and software tools used in this research.

\subsection{Common Factors of Preterm Birth}

There is often no known cause of spontaneous preterm birth but there are a multitude of factors which can lead to birth occurring at less than 37 weeks. Some of the medical factors can be preeclampsia and fetal distress, while some of the social factors can be stress and physical abuse. These factors can be grouped into three major areas leading to preterm birth: social stress and race, infection and inflammation, and genetics [28], [29]

\subsubsection{Social Stress and Race}

Several studies have shown a correlation between high rates of poverty and increasing rates of preterm birth [30]. Lack of access to healthcare and poor nutrition, as well as high rates of domestic abuse can be linked to poverty-stricken areas and these factors can negatively affect the health of both the baby and the mother. The rate of preterm birth amongst black women is generally higher in comparison to other races. In the United States, the rate of preterm birth in black women is twice as high as it is for white women [30]. Racial disparity in social situation and discrimination, which may lead to social stressors such as poverty and lack of access to proper healthcare, have been some of the reasons cited for this gap. 


\subsubsection{Infection and Inflammation}

Another key factor linked to high rates of preterm birth is intrauterine infection and inflammation. Bacterial infection can be widespread and can be found between the maternal tissues and fetal membranes, within the fetal membranes, within the placenta, within the amniotic fluid, within the umbilical cord, and within the fetus [30]. Bacterial infection often results in inflammation of the tissues and this response can trigger a premature labour and subsequent birth.

\subsubsection{Genetics}

There is some evidence that maternal genes have a large influence on the risk of preterm birth [30]. Therefore, one could review the family history of the mother to determine if relatives have had preterm births and this might be indicative of a predisposition to preterm birth. In addition, women who have had previous preterm births are at a higher risk for subsequent births to also occur prematurely [17].

\subsection{Cost of Preterm Birth}

The burden of premature births on health-care costs is significant. Patients born prematurely are hospitalized for longer, need to be monitored more regularly, and use more hospital equipment than full-term birth patients, as they are susceptible to a host of diseases and illnesses. Some of the medical devices often used patients born prematurely are incubators, multiple infusion pumps, invasive and non-invasive monitors, and ventilators. After discharge from hospital, premature infants are more likely to be re-hospitalized than full-term babies. It is estimated in Canada that the average hospital care cost for a preterm baby is nine times greater than a fullterm baby [31]. For full-term babies, it is estimated that they will remain in the hospital for around two days, whereas with preterm babies, the hospital stay may be as long as 104 days [31]. 
Due to these factors, it is estimated that the hospital care cost of a preterm baby in Canada may extend upwards to $\$ 117,000[31]$.

\subsection{Health of Preterm Infants}

Preterm delivery can result in the infant having several long-lasting disabilities. Premature infants have underdeveloped organs, specifically the lungs and heart. This can lead to severe neurological and cardiovascular problems. For instance, some infants can have respiratory distress, apnea and feeding problems; these illnesses all result in a longer hospitalization for the patient. One study showed that children at age eight who were born prematurely had more behavioural problems than their peers born full term [32]. Premature birth will likely impact the individual's life in the long term, with chronic lung disease and intellectual and developmental handicaps occurring commonly in those patients born most prematurely.

\subsection{Current Prediction Models}

\subsubsection{Cervical Length}

As previously stated, there is not one identifiable factor known to predict preterm birth; however, a correlation between the rate of shortening of the cervix and the prevalence of preterm birth has been observed. For instance, in one study, [33] researchers focused on women whose cervixes were shortening between 16-20 and 21-25 weeks and regularly observed the progression of their pregnancy. They found that if the cervical length was stable for periods of time and then would suddenly and rapidly decrease, this would often result in a preterm birth. Although this is an interesting finding, in practice it is difficult to observe patients sufficiently regularly to detect these changes and the detection methods are invasive and so a more realistic prediction model would be helpful in clinical work. 


\subsubsection{Uterine Electromyography}

Uterine Electromyography (EMG) is the practice of monitoring uterine contractility using electrodes placed on the uterus and can detect when there is increased contractility signaling the possible onset of preterm birth [34] With this method, the patient has to remain as still as possible when collecting these signals; if not, this can result in noisy signals which have to be filtered. In addition, the accuracy of this prediction model tends to be most accurate within a short window of labour (24 hrs to 4 days), similar to the fetal fibronectin test [34]. However, the focus of this thesis is to detect a preterm birth accurately, many weeks prior to labour, so that preventative care can be administered.

\subsubsection{Fetal Fibronectin Test}

The fetal fibronectin test has become the gold standard for predicting preterm birth. However, this test is expensive, invasive, and it is best designed as a short-term marker for preterm birth, as the sensitivity decreases from $71 \%, 67 \%$ and $59 \%$ within 7,14 and 21 days of delivery [35]. It is typically only measured after the membranes lining the uterus have ruptured, which is often the sign of impending preterm labour. Fetal fibronectin is a protein produced by fetal cells which forms a major portion of the maternal-fetal extracellular matrix [35]. Cervicovaginal leakage of this protein in the late second and early third trimester has been an indicator in many cases of spontaneous preterm birth [35]. The goal of this work, however, is to develop a tool that can be

applied non-invasively and throughout the early stages of pregnancy, before any signs of preterm labour develop. 


\subsubsection{Physician-Parent Decision Support (PPADS)}

The PPADS tool was developed in the MIRG lab at Carleton University and is a tool which provides shared decision-making between physicians and parents, concerning infants in the NICU [36]. The PPADS tool consists of two platforms: a clinician and a parent interface. The parent interface provides information about the infant with mortality risk estimations and provides a decision support module, allowing parents to communicate and understand the options available to them. The clinician interface contains the list of all patients, admission files and various medical details including outcome predictions. The PPADS system is currently being remodeled and a dictionary of medical terms will also help to enhance parents understanding of their child's condition.

\subsubsection{Ontario Perinatal Record}

Since 1997, The Ontario Antenatal Record consisted of a form which collected pregnancy data, and was administered by maternal care providers in Ontario. The Ontario Medical Association (OMA) had been the primary driver of distributing and updating this form. Recently a new partnership has arisen between the Provincial Council for Maternal Child Health (PCMCH), The Better Outcomes Registry \& Network (BORN) Ontario, the OMA and the Association of Ontario Midwives (AOM), to create an expanded scope of these forms called the Ontario Perinatal Record [37]. The questions within this form pertain to pregnancy, birth, and the early newborn period [37]. There are clinics in Ontario where this information is being entered electronically and one future method of monitoring patients for early risk of preterm birth would be to embed the tool developed in this research to be used in conjunction with the Ontario Perinatal Record to 
automatically screen the data as it is being collected and flag patients who are deemed to be at risk of preterm birth.

\subsubsection{Predictive Tools}

A preterm risk scoring tool is a means of risk assessment which contains many major or minor factors (previous preterm delivery, smoking/alcohol intake during pregnancy, etc...) and it estimates the likelihood of the outcome of a preterm birth [38]. Preterm risk scoring, and screening tools have been administered since the 1980s; however, the accuracy of these tests remains quite low, at around 17-38\% [38]. This can lead to a waste of hospital resources and therefore, a more effective and accurate system is needed that balances both high sensitivity and specificity metrics. One of the problems with current risk scoring tools is that they are often limited in their capabilities. This is related to the fact that currently there is no specific cause of spontaneous premature birth - just a multitude of factors which can contribute to a premature birth occurring. The advantage of using machine learning tools over risk scoring methods is the ability to easily analyze hundreds of possible preterm birth factors. The benefit of risk scoring systems is that they do identify the complex social and environmental factors which surround the risks of preterm births

\subsection{Summary of Previous Work}

\section{Catley}

The objective of Catley's thesis work was to develop an integrated hybrid classifier which combined ANNs (artificial neural networks) and MLP (multilayer perceptron)-ANNs with risk stratification. She also used case-based reasoning and a DT (decision tree) voting algorithm to predict preterm birth using an older version of the PRAMS database and the Perinatal Partnership Program of Eastern and Southerneastern Ontario (PPPESO) database (1999-2001). 
The results from this classifier yielded a sensitivity of $65 \%$ and a specificity of $84 \%$ and was validated with 9701 new patient cases. The data preparation methods used in this thesis work were to remove features with greater than $20 \%$ missing values and the $\mathrm{k}-\mathrm{NN}$ (k-nearest neighbor) CBR (case-based reasoning) algorithm, for imputing missing values [39].

\section{$\mathbf{Y u}$}

The objective of Yu's thesis was to combine an Artificial Neural Network and Decision Tree classifier: C4.5 DT Classifier [40] to output an integrated classifier to reduce the number of features and to increase the overall accuracy of the classifier. The model was validated using the PRAMS database and this integrated classifier could predict mortality rates with a sensitivity of $65 \%$ and a specificity of $84 \%$. The data preparation methods used in this thesis work were similar to Catley's: deletion of features with greater than $50 \%$ missing values, and the use of the k-NN CBR for imputation of missing values [27].

\section{Ong}

The objective of Ong's thesis was to improve the integrated classifier and to apply this classifier to two recently updated databases, (PRAMS and BORN) to predict mortality rates. This thesis also uses 5-by-2 cross validation to both ensure the model is trained with sufficient data and reduce overfitting. In addition, many more features were analyzed than in Yu's work, with factors obtained from four different types of cases: Parous, Nulliparous, Parous without Obvious clinical features, and Nulliparous without Obvious clinical features. The best performance metrics achieved was the PRAMS Parous dataset: 50\% for sensitivity and $92 \%$ for specificity when analyzing around 53 clinical features. The data preparation methods used in this thesis work were to remove outliers, deletion of features with greater than $50 \%$ missing values, deletion 
of cases with no outcome feature, and the use of the k-NN CBR as an imputation method for missing values [24].

\section{Other Research}

Research concerning predictive tools which use obstetrical data/devices and machine learning algorithms have been investigated. In work done by [41], this work consists of using uterine EMG data and artificial neural networks to classify preterm or term cases. The results were promising, the ANN was able to classify preterm cases with an accuracy of $92 \%$ and was able to classify term cases with an accuracy of $79 \%$. Also, in [42], the focus of this research was to document factors of importance by studying high-risk women from their first antenatal visit straight through to delivery. Researchers used logistic regression and artificial neural networks to identify significant risk factors (i.e. biochemical markers) which are associated with preterm birth. Finally, [43], also made use of the C5.0 DT classifier and ANN as machine learning tools, yet, the focus was on determining the top risk factors of preterm birth, in comparison to improving the sensitivity in this research. Factors such as maternal age, multiple births and maternal hypertension were just some of the factors which were identified to be of importance in predicting preterm birth. Predictive methods using machine learning algorithms are being studied extensively within the field of obstetrics, in search of faster, more accurate methods of predicting preterm birth.

\subsection{Review of Data Preparation}

There were two main areas to address in the data preparation stage; the presence of missing values and class imbalance in BORN and PRAMS.

\subsubsection{Missing Values}

There are three general categories of missing values [44]: 
1) Missing Completely at Random (MCAR)

2) Missing at Random (MAR)

3) Not Missing at Random (NMAR)

MCAR refers to random experimental error which affects the presence of an attribute; MAR refers to features which are not missing at random, but whose value depends on other measured features; NMAR refers to features not missing at random; the probability of this missing attribute depends on unavailable features. It is easier to impute missing values for MCAR and MAR variables, than NMAR [45].

When the probability that the data is missing, is the same for all features in the dataset (e.g. no blood pressure equipment to measure heart rate), this would fall under the category of MCAR. When the probability that the data that is missing is dependent on observed data (e.g. study on blood pressure, data on young people are less likely to be recorded, in comparison to older individuals because they do not attend clinics as often); this would fall under the category of MAR. Finally, when the probability that the data that is missing is dependent on data that has not been observed (e.g. individuals with lower incomes are often less likely to fill out information related to income), this represents NMAR [46]. As detailed in these examples, first-hand knowledge of the observed data is a key to making assumptions about features and which category the data falls under. The PRAMS dataset consists of survey data and BORN consists of automatically obtained data. Thus, there is little room for researchers to make assumptions because this data is obtained from external sources. 


\subsubsection{Discussion of Alternative Imputation Methods}

There are several methods for imputing missing values. Some of these methods have been analyzed below, to determine the best method of addressing missing values within the BORN and PRAMS datasets.

\subsubsection{Simple Imputation Methods}

There are simple imputation methods such as calculating the mean or mode of the feature to fill in missing values. However, calculating the mean or mode does not translate well for categorical features and ignores correlations between features within the clinical datasets [47].

\subsection{4. k-NN Algorithm}

In previous work [24], a k-NN algorithm was used for imputing missing values through a CBR tool developed in Microsoft Access. The k-NN algorithm makes two assumptions which make this algorithm ineffective for this research when compared to other imputation methods. The first assumption is that the data in the feature space are continuous [48]. Both the BORN and PRAMS datasets contain mixed type features (both categorical and nominal). Usually Euclidean distance is used as the distance function to measure differences between continuous features [48]. The second assumption is that the user must choose the k-value; this is usually done through cross validation [47]. The " $\mathrm{k}$ " value represents the number of neighbours which influence the classification. Difficulties related to these two assumptions were crucial in the decision to adopt another imputation method in this current research. There is a delicate balance between increasing the k-value, improving the accuracy and increasing the computational time. This is exemplified with Ong [24], where it was reported that it took up to three days to analyze these 
clinical datasets using this algorithm and the CBR tool. In addition, there are software programs (R) which drastically reduce the processing time from three days to hours.

\subsection{5. mice Algorithm}

The mice (Multivariate Imputation via Chained Equations) algorithm, as the name suggests, creates multiple imputations to reduce bias of results [49]. This algorithm was developed by Stef van Buuren and is a package in R. In the first step of the mice process, each missing value is temporarily set to the mean value within that feature. Then using one of the regression methods from the mice function (see Figure 2.1), which matches the data type of the feature, a missing value is obtained. This process is repeated as specified by the user; usually this cycle is repeated ten times [50]. The mice algorithm uses linear regression to predict nominal missing values and logistic regression for categorical missing values. The methods for the mice function are displayed below.

\begin{tabular}{lllc}
\hline Method & Description & Scale type & Default \\
\hline pmm & Predictive mean matching & numeric & Y \\
norm & Bayesian linear regression & numeric & \\
norm.nob & Linear regression, non-Bayesian & numeric & \\
mean & Unconditional mean imputation & numeric & \\
2L.norm & Two-level linear model & numeric & \\
logreg & Logistic regression & factor, 2 levels & Y \\
polyreg & Multinomial logit model & factor, >2 levels & Y \\
polr & Ordered logit model & ordered, >2 levels & Y \\
lda & Linear discriminant analysis & factor & \\
sample & Random sample from the observed data & any & \\
\hline
\end{tabular}

Figure 2.1. Regression methods in the mice algorithm to impute missing values [49] 


\subsubsection{Chosen Method: missForest Algorithm}

The missForest algorithm is a function which uses random forest classifiers to train each feature, and then this model is used to make predictions about missing values [48]. This algorithm was developed by Daniel Stekhoven and is a package in R. This function also provides an imputation error estimate for both the categorical and nominal data. Some papers show that missForest outperforms mice with a lower imputation error [47], [48]. In addition, with the mice algorithm, even though this algorithm has the capability to handle multiple types of data, one must make this explicit, coding in R. For instance, if one of the features in the dataset is numeric, then this had to be defined in the code as 'pmm' (predictive mean matching when using the mice algorithm). Similarly, when one of the features had two factors (i.e. "Yes" or "No") with two levels, this was defined to be 'logreg' for logistic regression, and if another feature had more than two levels, then this would be defined as 'polyreg' or multinomial logistic regression model. With many mixed types in the dataset, this process can become tedious. Similarly, to the k-NN algorithm, the number of imputed datasets with the mice model is controlled by the user.

Although the value of 10 seems to be the most widely chosen option, research has seen improvements in accuracy when this value is chosen to be anywhere up to 40 [50]. Therefore, again a trade-off between accuracy and computation time exists. The computation time using missForest in this research work was significantly faster than using mice, when the number of imputed datasets was chosen to be 10 . For instance, using missForest, the processing time took around 16 hours, while with mice repeating the process 10 times took around four days to complete. Also, as the mice algorithm is a multiple imputation method, this algorithm operates under the assumption of MAR (missing at random) [51]. However, there is a risk of biasing the results if this assumption is made without strong justification [51]. Since, missForest is a non- 
parametric algorithm, this removes the researcher from having to make incorrect assumptions about missing values within features.

\subsubsection{Class Imbalance}

Most medical data contains an imbalance of classes, with the disease case usually being the rare occurrence in a dataset [52]. This is exemplified in both the PRAMS and BORN datasets, where the preterm cases represent around $20 \%$ and $8 \%$ respectively. This creates imbalanced datasets which affects the classification accuracy during training and testing [53], [54].

\subsubsection{Discussion of Alternative Class Imbalance Methods}

If the dataset is not balanced during training, the classifier output could be biased, and the classifier could misclassify a preterm birth label as a term label. In this case the classifier views the small proportion of preterm labels as noise or outliers, in comparison to the larger set of term labels. Thus, the specificity metric of the classifier will be very high while the sensitivity will be low. It is necessary to balance the class labels so that the ANN classifier will be less biased [55]. In this research, it appears to be a more serious misclassification to falsely predict a preterm label as a term label, than a term label as a preterm label.

\subsubsection{Get more training cases}

Obtaining more training cases can be expensive and may be unavailable; it may not be possible for researchers to get more cases. In our research, related to time restrictions (i.e. preparing a dataset from BORN could take on average 6-8 months), it simply was not feasible or cost effective to request more preterm cases from the BORN and PRAMS datasets. It is always quite complicated to obtain ethics clearance to acquire new data. 


\subsubsection{Oversampling the minority class}

Oversampling the minority class would entail replicating the preterm cases. The disadvantage of this method lies in possible overfitting of the minority class, as there are many more samples created from replicating the minority cases [56], [57]. Also, with over 600,000 cases in the BORN dataset and over 100,000 cases in the PRAMS dataset, oversampling would significantly increase the size of these datasets; leading to increased computational time for training and testing the Artificial Neural Network classifier.

\subsubsection{Chosen Method: Undersampling the majority class}

Several papers have reported the benefit of undersampling over oversampling when dealing with class imbalances. Oversampling may result in over-fitting of the classifier and will result in longer training times due to the increase in the sample size [56]. Although the disadvantage with undersampling is the loss of "valuable" information, the focus of this research is on accurately predicting preterm cases. The most "valuable" information lies in the preterm cases. Reducing the number of term cases, greatly improved computational time and sensitivity results during the training and testing of the neural network classifier.

\subsection{Performance Metrics}

\subsubsection{Confusion Matrix (Contingency Table)}

The purpose of a confusion matrix is to showcase the predictions from a classification model versus the accurate predictions, to determine the efficiency of the model in predicting an outcome [58]. For instance, in Table 2.1., the positive column displays both the true predictions from the model output and the number of predictions the model "classified" as false predictions, but which were true. 
Table 2.1 2-by-2 Confusion Matrix

\begin{tabular}{|l|l|l|l|}
\hline & \multicolumn{3}{|c|}{ Actual Value } \\
\hline \multirow{4}{*}{ Predicted Value } & & Positive & Negative \\
\cline { 2 - 4 } & Positive & True Positive & False Positive \\
& & (TP) & $($ FP) \\
\cline { 2 - 4 } & Negative & False Negative & True Negative \\
& & $($ FN) & $(T N)$ \\
\hline
\end{tabular}

\subsubsection{Correct Classification Rate (CCR)}

This metric is a measure of the accuracy of the model in being able to predict cases [59].

$$
\text { Accuracy }=\frac{T P+T N}{T P+T N+F P+F N}
$$

\subsubsection{Misclassification Rate}

This metric is a measure of how often the model makes an incorrect prediction [59].

$$
\text { Missclassification Rate }=\frac{F P+F N}{T P+T N+F P+F N}
$$

\subsubsection{Sensitivity}

Sensitivity (True Positive Rate) is a specific parameter focusing on the ability of the classifier to accurately classify a case which is defined as positive [58]. For instance, a positive case can be defined as a patient having a preterm birth (or a specific disease). Therefore, if the sensitivity of your test is $100 \%$, this means the test will correctly label all patients who have preterm birth as preterm births. 


$$
\text { Sensitivity }=T P R=\frac{T P}{T P+F N}
$$

\subsubsection{Specificity}

Specificity is a specific parameter focusing on the ability of the classifier to accurately classify a case which is defined as negative [58]. Continuing with the same above example, if the negative case is defined as the patient having a full-term outcome, a specificity of $100 \%$ means that the test would correctly label all patients who have births to term as full-term outcomes.

$$
\text { Specificity }=T N R=\frac{T N}{T N+F P}=1-\text { False Positive Rate }(F P R)
$$

\subsubsection{F1-Score}

This score functions as a weighted average of the precision and recall. The closer the classifier's F1-score is to 1 , the higher the precision and recall values will be [60].

$$
F 1=\frac{2 T P}{2 T P+F P+F N}
$$

\subsubsection{Prevalence}

Prevalence is a measure of the prior probability of the individual having the disease before the model is tested given the population size [61]. It is an important measure for the MIRG group as it ensures clinical relevance and acts as a threshold. In the context of this research, prevalence would relate to the proportion of the population who have had a preterm birth. In Ontario, the prevalence of preterm birth is around $7.9 \%$ [16]. As a result, during the final testing stage, the test sets evaluated by the ANN will use the prevalence in the population. 


\subsubsection{Positive Predictive Value \& Negative Predictive Value}

The positive predictive measure is defined by the probability of truly having the disease, given a positive outcome from the test, whereas the negative predictive value is the probability of not having the disease given a negative outcome [58]. There is a direct correlation to the PPV and the prevalence, where if the prevalence is low the PPV will also decrease.

$$
\begin{gathered}
P P V=\frac{T P}{T P+F P} \times 100 \\
P P V=\frac{\text { Sensitivity } \times \text { Prevalence }}{\text { Sensitivity } \times \text { Prevalence }+(1-\text { Specificity }) \times(1-\text { Prevalence })} \times 100 \\
N P V=\frac{T N}{T N+F N} \times 100 \\
N P V=\frac{\text { Specificity } \times(1-\text { Prevalence })}{\text { Specificity } \times(1-\text { Prevalence })+(1-\text { Sensitivity }) \times \text { Prevalence }} \times 100
\end{gathered}
$$

\subsubsection{Receiver Operating Characteristic (ROC) Curve}

The ROC is a curve displaying the performance of the ANN classifier at all classification thresholds, the $\mathrm{x}$-axis is the sensitivity (true positive rate) and the y-axis is derived from 1specificity (false negative rate). This curve also displays the trade-off between sensitivity and specificity. In this research the purpose is to obtain classifier results which optimize sensitivity but also maintains a relatively high specificity. If the curve rises quickly in the beginning, this might indicate better classifier performance when comparing different ROC curves [58]. Figure 2.2. highlights the features of importance in the ROC curve [24]. 


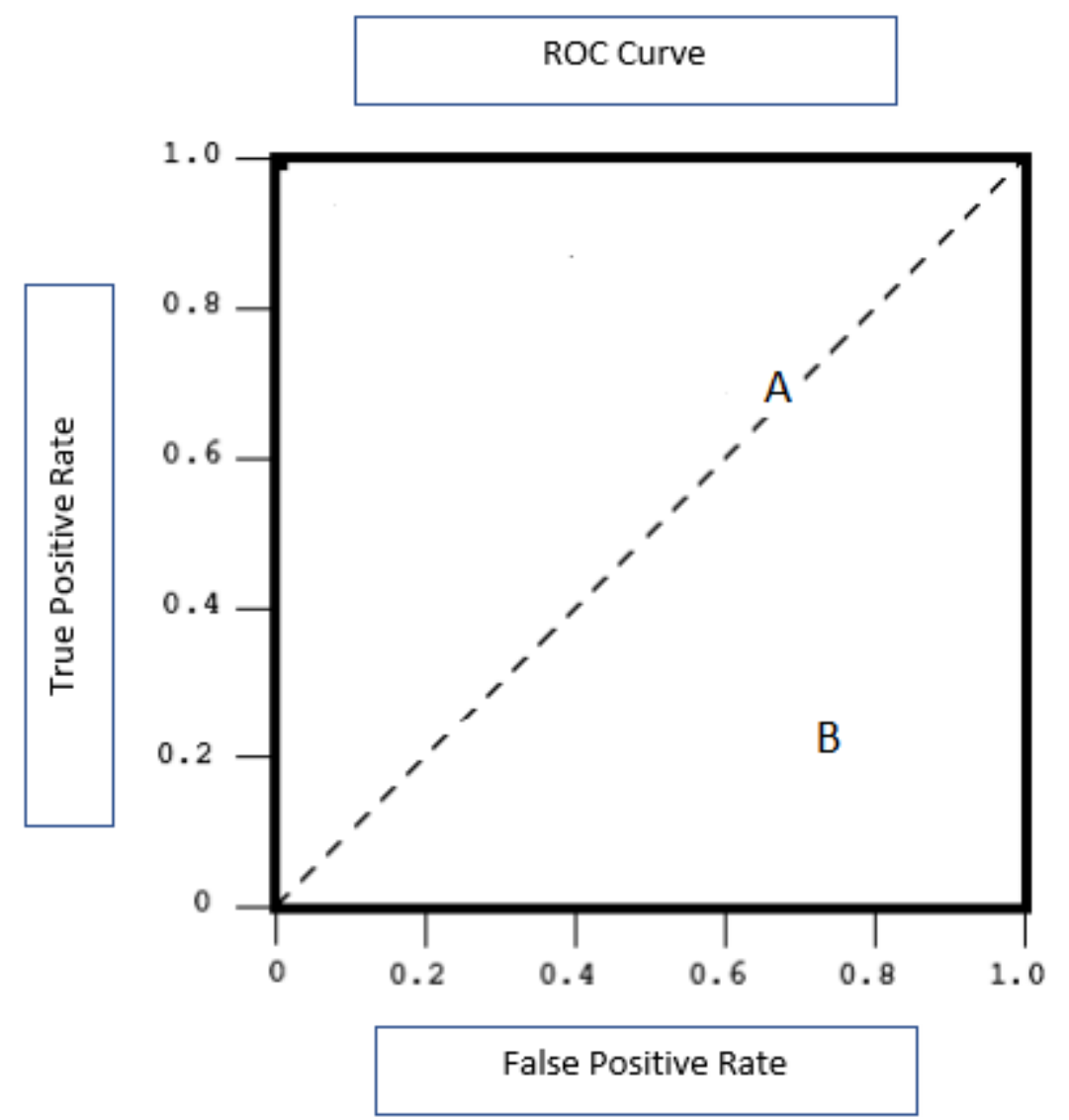

Figure 2.2. ROC curve and the different points of significance

- At coordinate $(0,0)$ there is no positive classification given and therefore no false positive classification exists. At point $(1,1)$, the classifier assigns a positive class label to all points hence it is where the false positive rate is at its max. At point $(0,1)$ the classifier has $100 \%$ sensitivity and specificity and represents perfect ability to discriminate between preterm and term cases

- A diagonal line (A) indicates random guessing. Points on this line indicate that TPR and FPR are equal thus classifier performance is random and does not contain useful information.

- Point $\mathrm{B}$, falling below the $\mathrm{A}$ line is worse than random guessing. May indicate that useful information is contained in the classifier however the application is incorrect. 


\subsubsection{Area Under the Curve (AUC)}

The AUC is a measure of how accurate the classifiers predictions are. An AUC value of 1 represents $100 \%$ accuracy in predicting preterm births, while an AUC accuracy of 0 represents $0 \%$ accuracy in predicting preterm births. An AUC value should strive to be above 0.5 , as 0.5 represents a classifier which is as good as random guessing. The effectiveness of this value is summarized in Table 2.2. [62].

Table 2.2 AUC Index and its Effectiveness labels

\begin{tabular}{|l|l|l|}
\hline Min & Max & Effectiveness \\
\hline & $\leq 0.5$ & No discrimination \\
\hline 0.5 & $<0.7$ & Poor discrimination \\
\hline 0.7 & 0.8 & Acceptable \\
\hline 0.8 & 0.9 & Excellent \\
\hline 0.9 & 1.0 & Outstanding \\
\hline
\end{tabular}

\subsubsection{Mathews Correlation Coefficient}

The Mathews Correlation Coefficient (MCC): is a correlation coefficient between the observed and predicted classifications. This metric varies between -1 and $1,-1$ indicates a completely wrong binary classifier, 0 represents an uncorrelated classifier (as good as random guessing) and 1 indicates a completely correct binary classifier [63].

$$
M C C=\frac{(T P \times T N)-(F P \times F N)}{\sqrt{(T P+F P)(T P+F N)(T N+F P)(T N+F N)}}
$$




\subsubsection{Normalization}

Normalization was an important preprocessing step before evaluating the data with the Artificial Neural Network, this was done using the modified Z-score transformation. Normalizing refers to scaling the data to fall within a certain range. The ANN deals with nominal data and the BORN and PRAMS data contains categorical and nominal data. Therefore, it was important to normalize the dataset so that all the features were in the same range and no specific feature was given more weight than others during the training stage. There are several methods to normalizing the data. Options include centering the data to have a mean of 0 or scaling the data by the standard deviation [64]. Past work has shown that the neural network works best when normalized between the range of $[-1,1],[13]$. The normalization process will be expanded on in greater detail in Chapter 3.

\subsection{Pattern Classification Methods}

Pattern classification methods have been used with fields such as, medical informatics, to classify and categorize large amounts of medical data and output clinical outcomes when faced with medical problems. The two types of pattern classification tools used within this thesis are: Decision Tree (DT) and Artificial Neural Network (ANN) classifiers. Specifically, a hybrid classifier which uses both elements from the DT (feature reduction) and the ANN classifier (model evaluation) are used to classify the preterm and term cases.

\subsubsection{Supervised Learning}

Supervised learning is a type of machine learning process which contains labels in the output variable and this differentiates this type of learning from unsupervised learning. Furthermore, supervised learning can be classified into two categories: regression and classification [65], [66]. 
A regression problem is described as having a numerical real value label such as "weight" and a classification problem is described as having a categorical output label such as "preterm". This work deals with supervised learning, as the main objective is to determine an accurate preterm birth outcome label, using an Artificial Neural Network classifier.

\subsubsection{Unsupervised Learning}

Conversely, unsupervised learning is a type of machine learning process which contains no output labels. Unsupervised learning can also be classified into two categories: clustering and associations. As the name suggests clustering refers to understanding how groups (clusters) respond to certain features in a given dataset. Association refers to what rules or relations one can make based on similarities between groups [65], [67].

\subsubsection{Semi-supervised Learning}

Semi-supervised learning takes aspects from both supervised and unsupervised learning. Semisupervised learning contains labeled and mostly unlabeled data. Given this mix of labels, one could approach the problem with both an unsupervised or supervised approach. For instance, if one wanted to learn about patterns and structure of the data, an unsupervised learning algorithm could be one option [68]. However, in this work, the method used on the data would be a supervised learning approach for the dataset, as the objective is to accurately predict the unlabeled data with the labeled data provided [69].

\subsection{Feature Reduction}

Data reduction is a large section of data preprocessing [70]. The "curse of dimensionality" refers to the situation where it is often beneficial to limit the number of features to maintain classifier performance [71], [70]. A decrease in classifier performance might be related to overfitting, 
when the classifier is provided with too many features. When the classifier sees new data that it previously has not been trained on, the classifier performs poorly. Thus, appropriate feature selection (FS) is important to maintaining a high accuracy for the ANN, (especially since this classifier has a non-linear decision boundary prone to overfitting, in comparison to other classifiers [72]). Currently there are hundreds of features in both the BORN and PRAMS datasets and, by pruning these features down to an optimal subset, one can obtain improved classifier performance; it makes sense not to use all the clinical features provided during training.

There are many FS (feature selection) methods to draw from, and these are the three main categories [10]:

1) Filter FS methods

2) Wrapper FS methods

3) Embedded FS methods.

In Method 1, the features selected are independent of the classifier; the features are ranked based on a specific statistical measure (i.e. entropy) and chosen based on rank; in Method 2 the features are dependent on the classifier; a subset of feature is chosen and evaluated on the classifier, and the subset of features with the best classifier performance is chosen. The final method is akin to Method 2; however, these methods are much faster than the wrapper methods. This feature selection method occurs during the learning process. 


\subsection{Machine Learning Tools}

\subsubsection{Decision Tree Classifier}

Decision Tree Classifiers are supervised classification methods where decision-based rules, determined by the features, are input into the classifier [73]. There are a variety of DT classifiers: ID3, C4.5 and C5.0 [74]. Figure 2.3 depicts the framework of a decision tree classifier. A DT classifier consists of nodes, branches and leaves, where the nodes represent the features, the branches represent the decision rule associated with the node and the leaf represents the terminal outcome (preterm or term in this case). To begin the classification, a statistical measure (i.e. entropy) is calculated for each feature and the feature with the highest value is chosen to be the root node; this process is repeated until the outcome is achieved [72] .

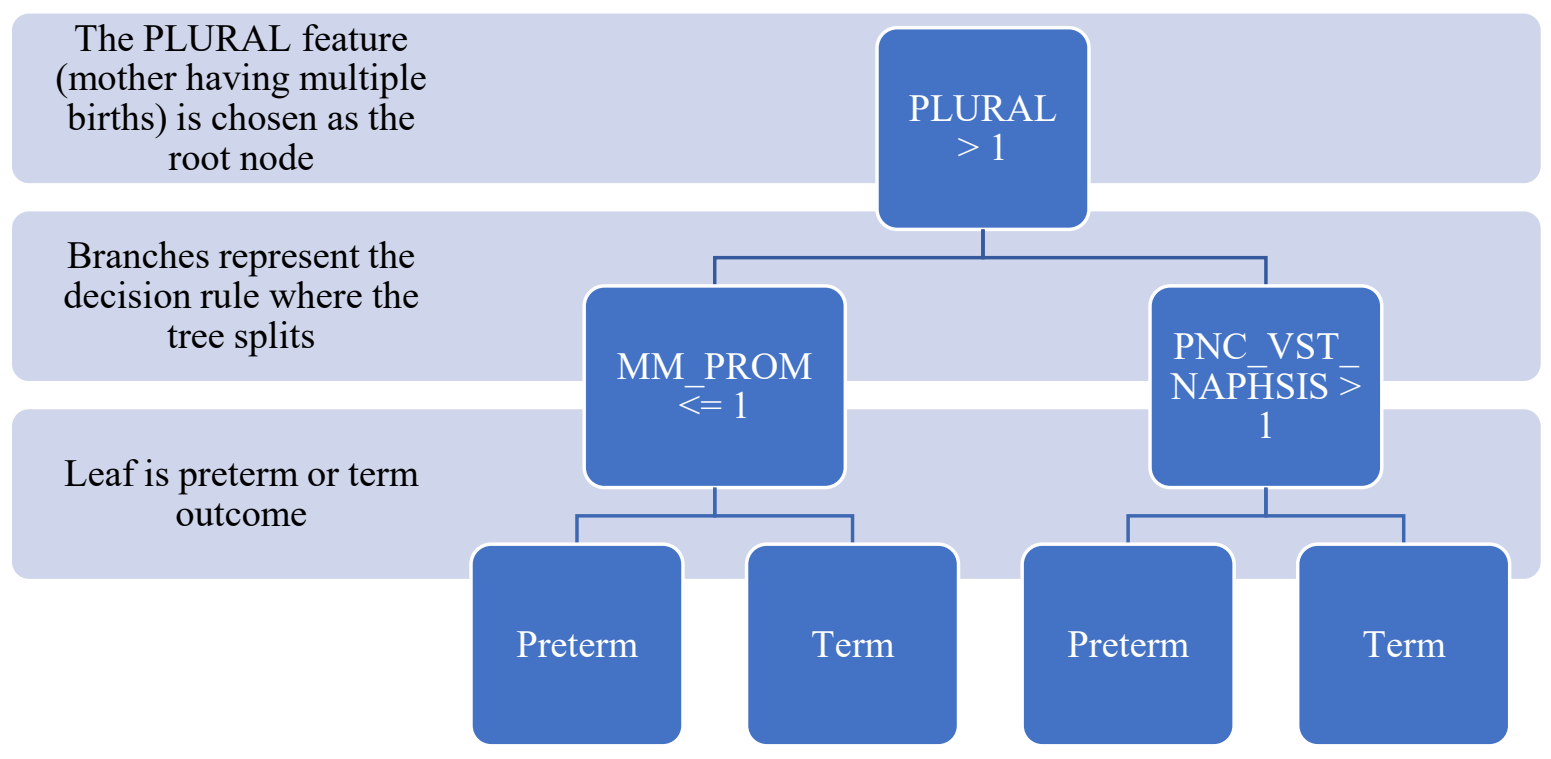

Figure 2.3 Depiction of the Decision Tree Classifier Framework [75] 
Some advantages to using DT classifiers include:

- The model is intuitive

- Data preprocessing is minimal (Decision Tree C5.0 can handle missing values)

Some disadvantages to using DT classifiers include:

- Risk of overfitting results

- In cases where the dataset has class imbalance, a biased tree can be produced

The disadvantages to using DT classifiers have been dealt with throughout this thesis work by firstly using cross validation, and secondly, by separating the validation set from the original dataset. This was done when testing the Artificial Neural Network (ANN) classifier, to reduce the risk of overfitting results. The second disadvantage of creating a biased tree was reduced by under-sampling the majority class of term cases by using the undersampling package in $\mathrm{R}$ [76]; these details will be expanded on later in the thesis.

\subsubsection{Random Forest Classifier}

The random forest classifiers can be described as a randomized ensemble of decision trees, as observed in Figure 2.4. The random forest classifier is a type of supervised learning algorithm which differs from decision tree classifiers. The DT classifier splits the features based on the most important statistical measure (i.e. entropy) in the feature subset; the random forest classifier splits features based on the most important statistical measure, derived from a random subset of features; this adds randomness to the model and reduces the correlation between trees. This creates a separate classification outcome for each tree, which is then aggregated, and a final vote is done [48], [77], [78]. 


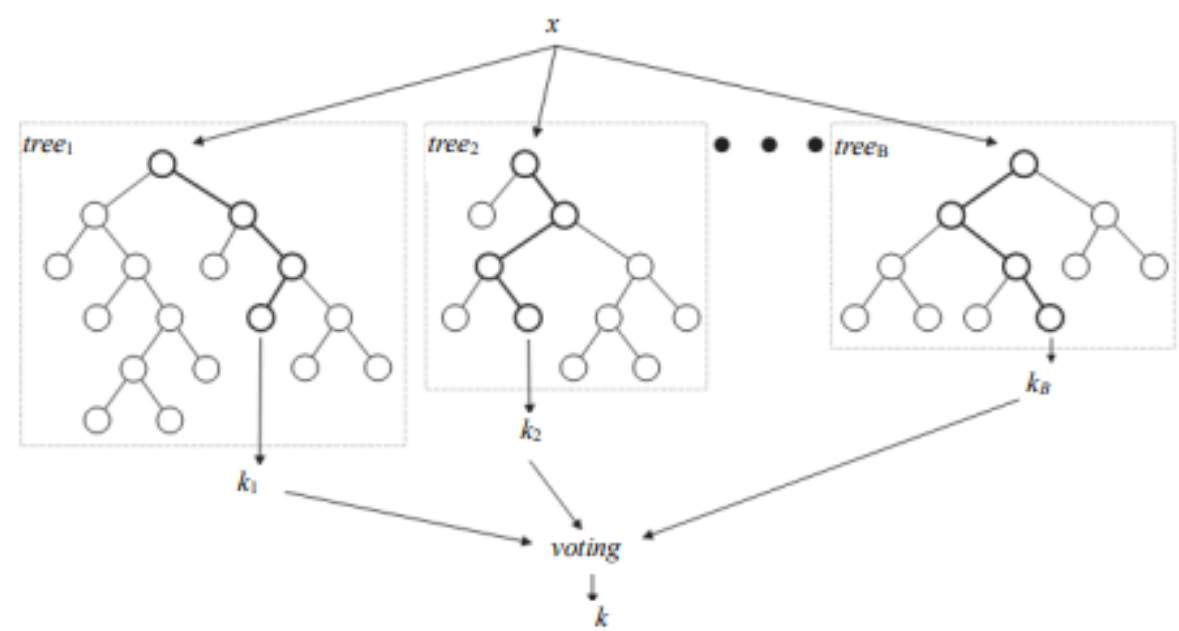

Figure 2.4. Depiction of the Random Forest Classifier Framework [79]

\subsubsection{Artificial Neural Networks}

Artificial Neural Networks were modeled to mimic processes within the brain. Artificial Neural Networks consist of mathematical algorithms which have a similar basic framework (see Figure 2.5): inputs are multiplied by a weight (this is assigned based on relative importance in comparison to other inputs) in the Input layer and passes into an activation function in the Hidden layer which produces an output (preterm or term) in the Output layer. Then, Input 1 is multiplied by the weight (-0.14) along with the other inputs and weights, is summed and the activation function is present in the Hidden layer, resulting in an Output value of 1 . There are several types of ANNs; some of the formulations include multilayer perceptron (MLP) and Radial Basis Function Networks (RBFNs) [70]. The specific activation function used in this research work was a sigmoid symmetric function (also known as the tanh function) which gives an output between $[-1,1]$ (see Figure 2.5), this is one of the most widely used functions for layered feed-forward networks [7], [80]. Other non-linear functions are the ReLU (Rectified Linear Unit) activation function which thresholds the output at 0 and replaces negative values 
with 0 . The output from the classifier is compared to known cases and adjusted by repeating this entire process again until a minimum error rate is achieved [70].

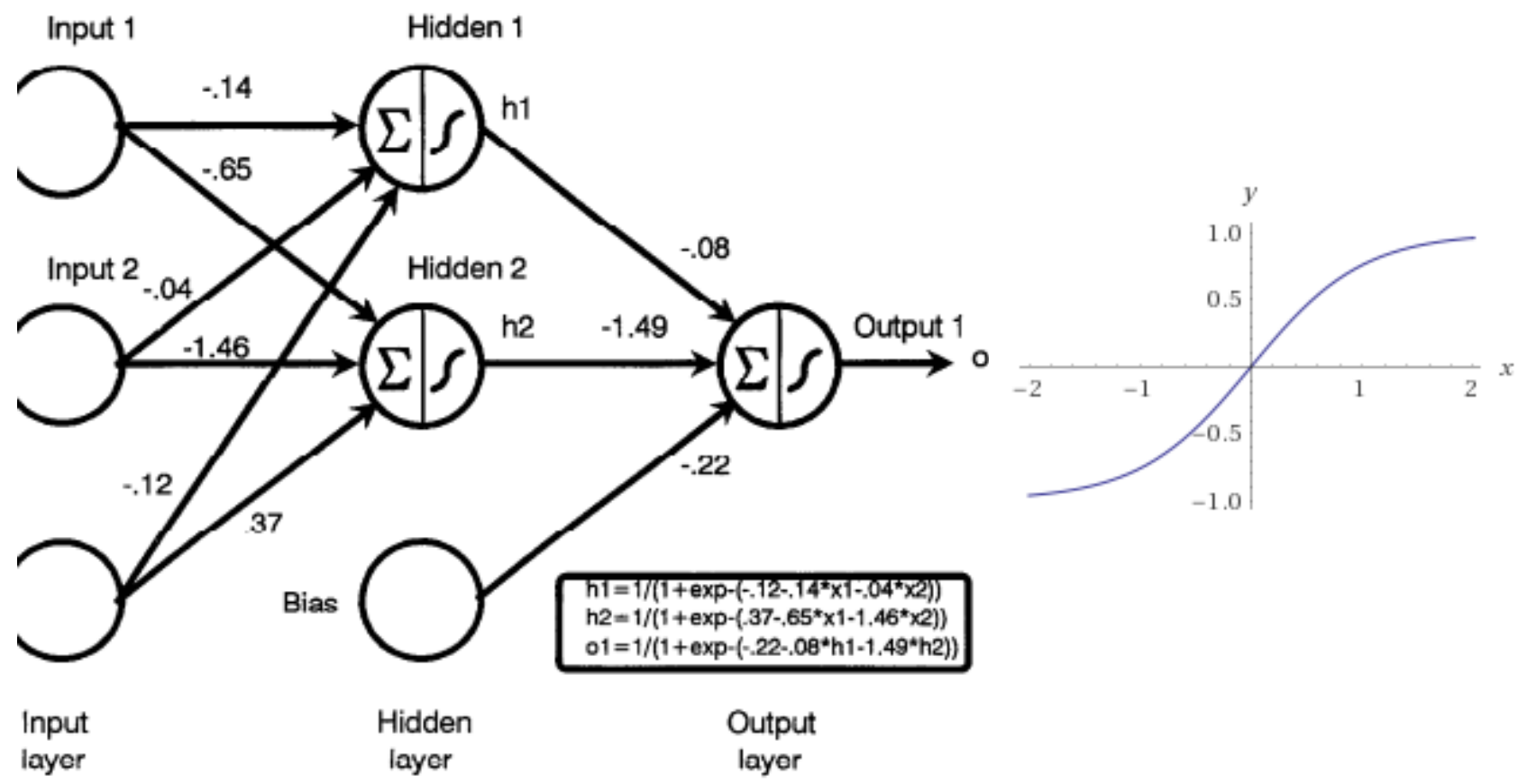

Figure 2.5. Depiction of the Activation Function and Artificial Neural Network Framework [81]

Some advantages of using ANN classifiers include:

- Performs well with regards to non-linear models

- Ability to learn models in real time

Some disadvantages of using ANN classifiers include:

- Sensitive to scaling features

- Does not do well with missing values

The disadvantages of using ANN classifiers have been addressed throughout this thesis work by normalizing the dataset before applying the ANN classifier and using the missForest package in $\mathrm{R}$ [48], to deal with missing values in both the BORN and PRAMS datasets. 


\subsection{Software Tools Used in This Research}

\subsection{1. $\quad$ R}

$\mathrm{R}$ (1993) is a statistical programming language which was created by Ross Ihaka and Robert Gentleman at the University of Auckland, New Zealand. $\mathrm{R}$ is a versatile program which is open source and can be integrated into several different platforms. R also contains several packages produced by academics and data scientists, some of which have been used for data cleaning in this research work to deal with missing values and class imbalance. Packages (missForest and ubUnder) were tested to determine which one offered the best fit with the clinical data provided [48], [76].

\subsubsection{Tableau}

Tableau software (2003) is a tool developed by Pat Hanrahan, Christian Chabot and Chris Stolte, which allows for data visualization [82]. This program was instrumental in transforming the raw data into informative graphics. The benefit of this approach was to be able to see which of the over 100 clinical features present in these two clinical datasets, (BORN and PRAMS), were strongly correlated with a preterm or a term outcome, so that further statistical analysis could be carried out in R. Tableau offers many different charts and diagrams such as: pie charts, geographical maps and bar charts. The ability in Tableau to easily display plots and graphs was important in communicating project goals.

\subsubsection{Cygwin Terminal}

The Cygwin terminal was used in this research to run several Bourne-Again-Shell (BASH) scripts for the C5.0 DT classifier and ANN classifier. This software provides a Unix-like environment and is an open source platform [83]. 


\subsubsection{See5/C5.0 Decision Tree Classifier}

See5/C5.0 is a data mining tool developed by (C) Rulequest Research 1997 [84]. The C5.0

decision tree classifier is an updated version of the popular C4.5 decision tree classifier [74].

Some of the improvements in the latest version are the ability to deal with noisy or missing data, boosting (that is using multiple decision tree classifiers for improved accuracy) and the ability to predict which features are important. The last point was of great significance to this research work. There are over 200 features in the PRAMS dataset and over 300 features in the BORN dataset; these datasets are focused primarily on maternal health factors; thus, there are many factors which are not directly related to predicting premature birth. Obtaining a set of features which contains only those features that are relevant to preterm birth reduces computational time, noise, the "curse of dimensionality" and subsequently increases the accuracy of the ANN. Decision trees are often used for feature selection because they display a good balance of high computational speed and high performance of the selected feature subset [10]. Removing irrelevant features will improve the accuracy and speed of predicting premature births when this adjusted data is inputted into the Artificial Neural Network (ANN) model. The C5.0 algorithm can handle missing values and displays the percentage attribute usage. This tells the user how important some features are in predicting a preterm birth outcome. In addition, this algorithm incorporates adaptive boosting. In this research, ten DT classifiers were generated instead of one, and each classifier voted for the predicted class (preterm or term); the votes were counted to determine the final class. This feature incorporated into the $\mathrm{C} 5.0$ algorithm, reduces the error rate significantly, instead of relying on one single classifier. 


\subsubsection{Fast Artificial Neural Network Library}

In previous work done by Catley [39] and Yu [27] the ANN was created using MATLAB software; however, MATLAB's Neural Network Toolbox is not open source software and there was difficulty in integrating it manually into the real time PPADS system. Previous work [85] focused on implementing a multilayer feed-forward-backpropagation ANN. Previous students have improved the ANN architecture through the years. The ANN-RFW (Artificial Neural Network Research Framework) developed by Rybchynski [86] was intended to improve automation and increase the prediction ability of ANNs. The problem with the use of this ANN is that it is difficult to integrate this classifier into the clinical environment; we wanted to use an ANN classifier which could quickly analyze large sets of clinical data.

The solution was to use the FANN (Fast Artificial Neural Network) Library to develop an ANN for classification purposes [7]. The advantage of using this library is that it has access to feedforward ANNs and the library is based on the C language which makes the FANN library easy to integrate with many different software environments. Also, the FANN library allows the user to easily manipulate the same ANN parameters used in past work [86]. In addition, the FANN library has access to feed-forward networks and these networks have superior computation abilities - which is critical for processing large amounts of medical data. Neural networks are also beneficial in identifying patterns and in identifying which trends deviate from these patterns. Articles have reported the benefits of using neural networks focused on classification problems and more specifically in the medical industry, such as with medical imaging [87], [88]. The ANN Builder can be run in several different modes: FAST, MEDIUM and SLOW. The FAST mode analyzes around $0.5 \%$ of all neural network combinations, MEDIUM mode 
analyzes around $7 \%$ of all options, and SLOW runs through all possible combinations. This feedforward artificial neural network is also multilayer, indicating an initial input phase, a defined number of hidden layers, and ending with an output layer. There are three phases for these neural networks (NN): a training, testing, and validation phase. The training and testing phases consist of feeding the NN both preterm and term cases, so it can learn to differentiate between these two classifications. The validation phase consists of inputting the $\mathrm{NN}$ cases that it has not previously seen (unlabeled data) and it outputs final classification labels as either preterm or term. There is user flexibility involved with this software; factors such as the learning rate, the activation function, the steepness value of the activation function and the values of the initial weight of the neural network can be manipulated by the user.

15 network parameters available through the FANN library [7] are listed below:

\section{Connection rate}

2. Number of hidden layers

3. Number of hidden nodes: Defines the number of nodes in each hidden layer

4. Connection weights: Two options available- randomly assign values to weights or initialize weights (Widrow-Nguyen algorithm)

5. Activation functions: There are six activation functions available- Sigmoid symmetric, Gaussian symmetric, Elliot symmetric, Linear piece symmetric, Sine symmetric, Cosine symmetric; all these functions output a value between -1 to 1 .

6. Activation steepness: Determines the speed at which the activation function goes from the minimum to the maximum.

7. Training algorithms: Four training algorithms available- Incremental training, Batch training, Quickprop training, Rprop training 
8. Learning rate: Determines the speed at which the network attains a minimum in the criterion function.

9. Training error function: Two error functions are available: A standard linear function or a hyperbolic tangent error function.

10. Incremental training momentum: This parameter speeds up the training y adding a proportion of the previous weight-change value to the new value

11. Quickprop training weight decay factor: Determines how much the weights need to be penalized to make sure they do not become too high during training.

12. Quickprop training maximum growth factor: Restricts the size of weights' growth

13. Rprop intial step-size: Determines the initial step-size for weights

14. Rprop step-increase: Determines how much the step size can increase during training.

15. Rprop step-decrease: Determines how much the step size can decrease during training. 


\section{Chapter: Methodology}

The main goal of this thesis is to improve the accuracy of the prediction tool in classifying preterm birth. Specifically, this thesis focuses on data preprocessing methods, to ensure the data is of the highest quality, before applying the Artificial Neural Network classifier for model evaluation. This chapter outlines the steps taken to achieve this goal.

The 8-step methodology for this work is outlined in Figure 3.1. Excluding Step 1, 4 and 5, this methodology followed closely with the work done by Ong [24]. This was done to accurately measure the effectiveness of the application of these data preparation tools, (Step $4 \& 5$ ) with the overall improvement of the classifier's ability to predict preterm birth.

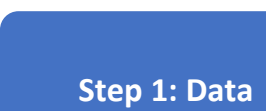

visualization
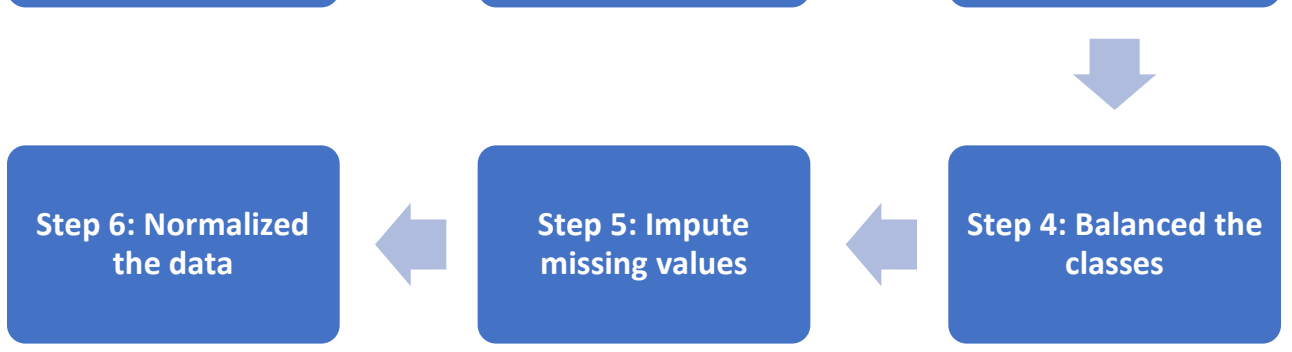

\section{Step 3: Choosing}

features with

greater than $\mathbf{5 0 \%}$

importance

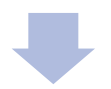

Step 7: Divided into

test, train and

validation sets

Step 8: Execution of

the ANN Builder

Figure 3.1. Schematic representation of the methodology used for the preterm birth classification

tool 
A brief description of each of the 8 steps followed is outlined in Table 3.1.

Table 3.1. Methodology for the development and evaluation of the predictive tool

\begin{tabular}{|l|l|}
\hline \multicolumn{1}{|c|}{ Steps } & \multicolumn{1}{|c|}{ Description } \\
\hline Step 1: Data visualization & $\begin{array}{l}\text { This step was done to increase our knowledge of the features } \\
\text { and missing values included in BORN and PRAMS, through } \\
\text { visualization and statistical understanding. }\end{array}$ \\
\hline Step 2: Eliminated cases and features & This step was done following the methodology done in past \\
50\% importance using the C5.0 DT classifier & $\begin{array}{l}\text { This step involved feature reduction of the numerous } \\
\text { Step 3: Choosing features with greater thanded in BORN and PRAMS }\end{array}$ \\
\hline Step 4: Balanced the classes & This step involved balancing the number of preterm and \\
\hline Step 5: Impute missing values & term cases for training of the ANN. \\
\hline Step 8: Execution of the ANN Builder & This step involves filling in the missing values with the \\
reduced dataset from both BORN and PRAMS
\end{tabular}




\subsection{Preliminary step: Ethics Clearance}

This thesis work was approved by the Carleton University Research Ethics Committee and by the CHEO Research Ethics Board. The databases used in this thesis work were: (1) Better Outcomes Registry \& Network (2010 - 2012) database (BORN) and the (2) Pregnancy Risk Assessment Monitoring System (2009-2011) (PRAMS). A contract was signed protecting the intellectual property of the data and their confidentiality before accessing both databases for this thesis.

\subsection{Step 1: Data Visualization}

As noted in Chapter 2, data visualizations can be useful in determining possible relationships between attributes and can provide information about individual attributes. Data visualization can also reveal noisy aspects of the data such as outliers and show clusters in the data which might indicate which is the best classifier to model the data.

The benefit of using Tableau as an analytic tool is that you can transform your data from raw values to informative graphs, such as bar charts. Bar charts were widely used for data analysis in this work because bar charts are good for showing comparisons. R was also used to visualize the missing values present in BORN and PRAMS. Before applying complex algorithms, it is important to understand the data and features present.

\subsection{Step 2: Eliminating cases and features}

The first step in the removal of features was to load the raw clinical data into Excel. Any missing values were denoted as 'N/A'. Then the 'COUNTIF' formula was used in Excel to count the number of cells which contain missing values; from this, one could determine the percentage of missing values for each feature. All features which contained greater than $50 \%$ of missing values were removed. This followed steps taken in the theses of Ong [24] and $\mathrm{Yu}$ [27]. Applying 
the Artificial Neural Network classifier in the final stage would require the datasets to have no missing values; the focus was to not introduce too much imputed data into the dataset, to reduce bias of the data. In addition, any cases which had a missing outcome (preterm or term) were deleted. A more detailed description of the removed features can be found in Appendix B.

It was important to only included Parous and Nulliparous features which could be known at 23 weeks gestation so that preventative treatment could be applied, and patients could be monitored to improve clinical outcomes. Both the BORN and PRAMS dataset were further divided into BORN_Parous, BORN_Nulliparous, PRAMS_Parous and PRAMS_Nulliparous datasets based on features selected by our clinical supervisor. Features which were only specific to mothers, who have had previous births, fell under the Parous category (i.e. previous premature birth). After feature and case elimination steps were applied to the raw BORN and PRAMS data, divisions between BORN_Nulliparous, BORN_Parous, PRAMS_Nulliparous and PRAMS_Parous were applied in the subsequent steps.

\subsection{Step 3: Choosing features with greater than $50 \%$ importance using the C5.0 DT classifier}

The C5.0 DT classifier has the capability to deal with missing values. Therefore, two options needed to be investigated:

1. Balance the data (using the ubBalance package in $\mathrm{R}$ ), impute the missing values (using the missForest package in R), and then apply the C5.0 DT classifier for feature reduction 
2. Apply the C5.0 DT classifier for feature reduction with the missing values present in BORN and PRAMS, and then carry out the preprocessing steps (balance the data and fill in missing values)

The results are summarized in the next chapter.

1. The first step in creating the C5.0 DT classifiers was to modify a file called "mortality.names" this file contained information about the features and classes (files are labeled as mortality/nonmortality throughout this research, due to past work done by Hasmik on the ANN Builder [13], her work was focused on neonatal mortality risk estimation models using Artificial Neural Networks)

2. In the mortality.names file the OUTCOME feature represented the target attribute, the CASE ID was the label attribute and the rest of the features in: PRAMS_Parous, PRAMS_Nulliparous, BORN_Parous or BORN_Nulliparous were defined to be continuous (numeric) or discrete (nominal).

3. The next step was to create two csv files, one labeled mortality.csv and the other labeled nonmortality.csv. The first file contained all the cases with a preterm label and the other file contained all the cases with a term label.

4. After this was done, a script called./create_5by2_folds.sh along with the mortality and nonmortality files were executed in the Cygwin terminal. This script automatically created 10, 5-by-2 cross validation sets.

5. The next step to create the DTs is the bash script./run_dt_5by2.sh. This is a command which calls C5.0 to create DTs for each of the 10 previously created sets. This produced 10 output files. The decision trees can be viewed in the mortality_fold_1_a.out and the mortality_fold_1_a.out.boost (boosting enabled) files (see Figure 3.2). 


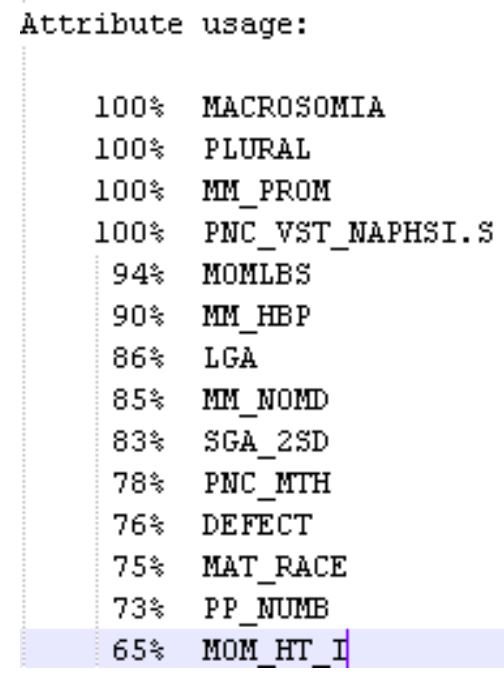

Figure 3.2. Script files representing the DT classifiers

6. Within each of the script files the percentage usage of each feature is listed (See Figure $3.3)$.

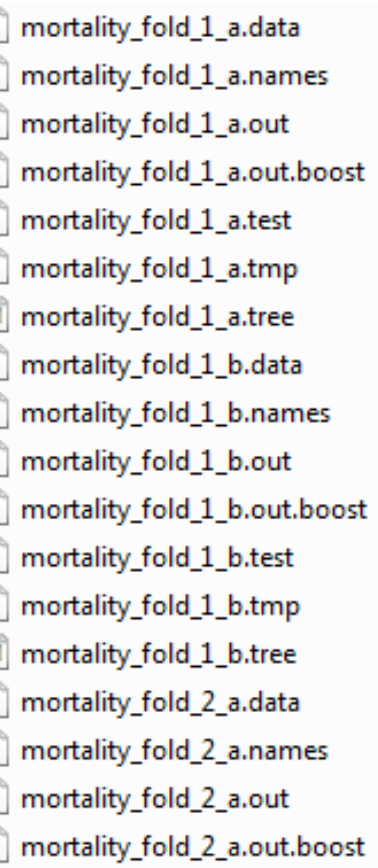

Figure 3.3. Feature percentage usage displayed

7. Across the 10, C5.0 DTs, features with high attribute percentage were repeated; the variance existed close to the threshold of $50 \%$. The final feature subsets were chosen by averaging the percentage usage of each feature across the 10 trees and choosing features which had the highest averages and exceeded the 50\% threshold. The results of the final feature subset for each of the four datasets can be found in the next chapter. 


\subsection{Step 4: Balancing the classes}

In this research work, the unbalanced algorithm was used to apply the random undersampling technique to the clinical datasets, in R [76]. The undersampling sub-function randomly removes instances of the majority class while leaving all instances of the minority class (ubUnder package). The ubUnder type has to be selected because the ubBalance package contains both undersampling and oversampling methods. This function allows the user to determine which percentage of the majority class will be left after sampling. In this work, $50 \%$ preterm cases and $50 \%$ term cases remained in the training dataset, so the classifier could be trained with an equal proportion of both classes. The code below describes the BORN or PRAMS dataset represented as $\mathrm{X}$, with the $\mathrm{Y}$ variable representing the final OUTCOME feature (preterm or term cases) as well as the type of balancing method (ubUnder) and the sampling parameters. The description of which parameters were selected from this function are described in Table 3.2.

balance_dataset $\leftarrow$ ubBalance $(X, Y$, type="ubUnder", positive= 0 , perc $=50$, method="percPos", w=NULL, verbose=FALSE)

Table 3.2. Description of parameters for package in $R$ (ubBalance)

\begin{tabular}{|l}
\hline X represents the BORN or PRAMS \\
Y represents the OUTCOME feature (preterm or term) in BORN or PRAMS, which must be a \\
binary factor \\
type represents the balancing technique, in this research $u b U n d e r$ was used, to remove \\
instances of the majority class (term) \\
\hline positive represents the majority class (term cases), all term labels were changed from -1 to 0, \\
when using this function
\end{tabular}


perc represents the sampling percentage which was set to 50

method represents setting the percentage of positives to $50 \%$ after undersampling

w represents sampling the majority instances with equal weights, when w is set to NULL

verbose represents not printing extra information, when set to FALSE

\subsection{Step 5: Input missing values}

Before applying the ANN classifier, it was necessary to impute the missing values found within the BORN and PRAMS dataset. Random forest classifiers were used to make predictions on missing values in the BORN and PRAMS datasets. This was done by initially replacing the missing values with the mean of the non-missing values within each feature. All features were then sorted from lowest to highest, according to the amount of missing values. Then each feature was trained with a random forest algorithm and predictions were made on the missing values. This process was iterated until the difference between the previous and the new imputed matrix increased for the first time. The package used to impute the missing values was missForest [48]. The code below described inputting the BORN or PRAMS dataset with missing values (noNAs file) with the missForest algorithm applied. Then the filled in data was written to a text file once the process ended. The description of which parameters were selected from this function are described in Table 3.3.

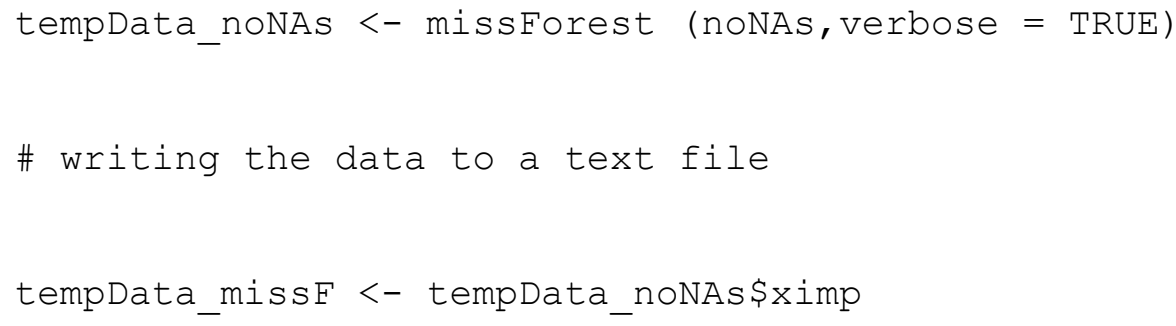


write.table (tempData_missF,

"C:/MIRG/Thesis2017/missForestResults.txt", sep="\t")

Table 3.3. Description of parameters for package in $R$ (missForest)

noNAs represents the BORN or PRAMS dataset with missing values

verbose represents additional output between iterations: estimated imputation error and runtime, when TRUE

\subsection{Step 6: Normalizing the data}

Once the above preprocessing steps were applied, the four datasets were prepared for model evaluation using the MIRG ANN classifier. Before applying this classifier, normalizing the data was done, as the ANN tends to perform better when large range differences amongst features are minimized [89]. In this work, the modified Z-score transformation equation was used to transform the values between the range of -1 and 1, based on the MIRG ANN Guide [24].

The data was normalized to fall between $[-1,1]$ so that the activation function in the ANN treats all features weighted equally during training. Normalization of the training, testing and validation data was automated using BASH scripts. For scaling and normalization purposes, the modified z-score transformation has been used previously in the MIRG lab [90], which scales the data between -1 and 1 is:

$$
\hat{x}_{i}^{n}=\frac{x_{i}^{n}-u_{i}}{3 \sigma_{i}}
$$

Where $x_{i}$ is the feature of interest, $u_{i}$ is defined as the population mean for each feature and $\sigma_{i}$ is defined as the population standard deviation for each feature.

This automation was done using the following steps: 
a. Using SQL queries, the mean and standard deviation was obtained for all attributes in the BORN Parous, BORN Nulliparous, PRAMS Parous and PRAMS Nulliparous features. The mean and standard deviation values were written to a file named "normalization.csv".

b. Each dataset (BORN_Parous, BORN_Nulliparous, PRAMS_Parous and PRAMS_Nulliparous) was divided into files called "mortality" and "nonmortality" csv files

c. The mortality.csv file contained the balanced preterm cases with missing values imputed and the nonmortality.csv files contained the balanced term cases with missing values imputed.

d. Most of the values within each feature fell between the ranges of 1 to 5 as many of the features within these datasets were categorical, however, some of the continuous features such as maternal age had values of greater than 40 . When looking at the dataset as a whole these values might be considered as outliers, however, the information within these features is valuable for predicting preterm birth. Therefore, normalization was done column-wise for each feature to ensure that the values fit the range of - 1 to 1 and all values were included and not dropped.

e. A temporary file was created for both the mortality and the nonmortality cases. The modified z-score was calculated for each attribute column in mortality and nonmortality. This was done by obtaining the mean in the first row of the attribute column and then the second row contained the standard deviation for that attribute column. Once these two statistics were 
obtained, using equation (1) above, the column data was normalized. In these temporary files, both the Case ID and Outcome features were excluded because these values should not be normalized.

f. Once all the feature columns were normalized, the data were combined into a single csv file with the untouched Case ID and Outcome features.

\subsection{Step 7: Divide into train, test and verification sets}

There are three divisions of the data which are defined. First, the training set is the data that trains the classifier, to improve the overall accuracy of classifying preterm and term cases. The test set is the data which is not a part of the training set and is tested by the classifier during model evaluation. Lastly, the verification set is the data which is unseen before inputting the data into the neural network model (data preprocessing is still applied to these cases). The purpose of this verification set is to output the true performance of the classifier, with data that it has not been trained or tested on, to minimize overfitting. These three sets were created using 5-by-2 Cross Validation which is described below in Table 3.4. Separate from these three divisions, a final test of the neural networks was evaluated on validation sets which consisted of unlabeled data and contained $7.9 \%$ prevalence to match the population.

Table 3.4. Division of train, test and verification sets

\begin{tabular}{|l|l|l|}
\hline Training Set & Testing Set & Verification Set \\
\hline $50 \%$ of the dataset & $25 \%$ of the dataset & $25 \%$ of the dataset \\
\hline
\end{tabular}

\subsubsection{5-by-2 Cross Validation}

This work used 5-by-2 Cross Validation, to reduce overfitting of the classifier during the training stage. Also, creating 10 sets of training, test and verification data with an equal number of 
preterm and term cases; to ensure sufficient data was being verified by the ANN model. Before using the ANN classifier, the clinical datasets were first normalized using the modified z-score formula. BASH files have been developed by Gilchrist [91] to carry out 5-by-2 cross validation. A BASH script file called create_5_by_2.sh carried out the following steps:

PHASE 1: There are two files one which contains the preterm cases and another file which contains the term cases.

PHASE 2: Preterm and term cases are randomized and divided between Set A and Set B. At this point the preterm and term cases remain separate.

PHASE 3: Set A which contains both preterm and term cases is further divided between two cases (A1 and A2) while Set B remained unchanged. At this point A1, A2 and B contain preterm and term cases. The subset of data is now $50 \%$ training, $25 \%$ test and $25 \%$ verification.

PHASE 4: The above process is reversed where Set B (containing preterm and term cases) is further divided between two cases (B1 and B2) while Set A remains unchanged and the same ratio is present.

PHASE 5: As a result of PHASE 3, Set \#1 is produced which consists of $\mathrm{B}, \mathrm{A} 1$, and $\mathrm{A} 2$ for the training, test and the verification set respectively. While PHASE 4 results in Set \#2 which consists of A, B1 and B2. This process is repeated five times to create a total of 10 sets of training, test and verification data 
These steps ensure preterm and term cases are divided into equal parts and therefore there exists an equal number of preterm and term cases for train, test and verification of the DT and ANN models. These steps are summarized in Figure 3.4.

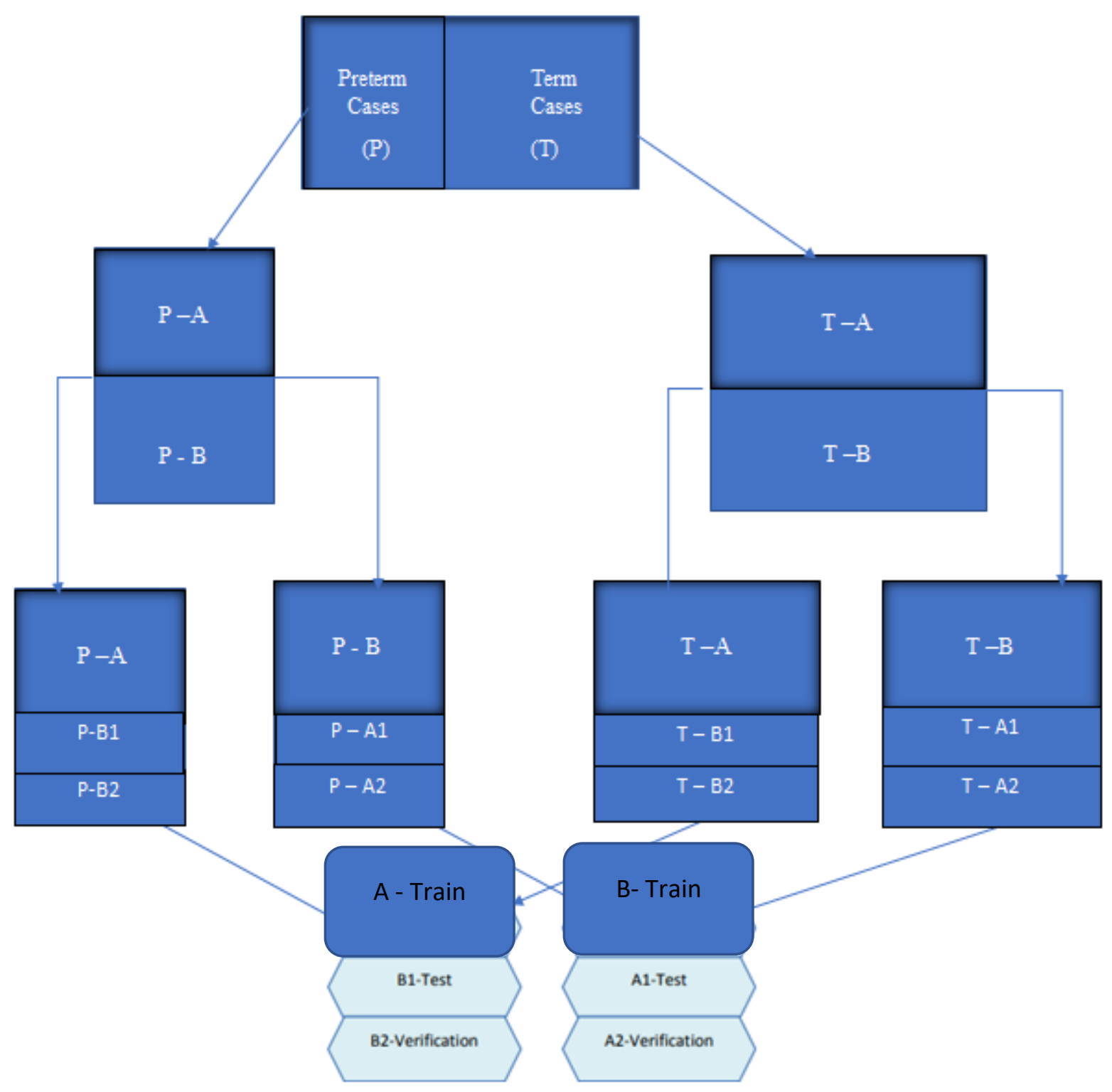

Figure 3.4. 5-by-2 Cross Validation to create train, test and verification sets 


\subsection{Step 8: Execution of the ANN Builder}

The purpose of this research was to follow similar steps applied, in the methodology of previous work, to compare the difference in sensitivity results for preterm birth predictions when focusing on data preparation. Therefore, the same ANN Builder using the FANN library, was used from past research [7].

1. These steps were repeated for all four datasets.

2. The ANN Builder software was used to run the ANN Classifier and carried out training and testing of the data.

3. The following network structures were automatically detected by the software for training (see Figure 3.5): the number of hidden layers, the number of inputs (this is dependent on the number of features determined by the C5.0 Decision Tree Classifier) and the number of outputs is 1 (term or preterm). 


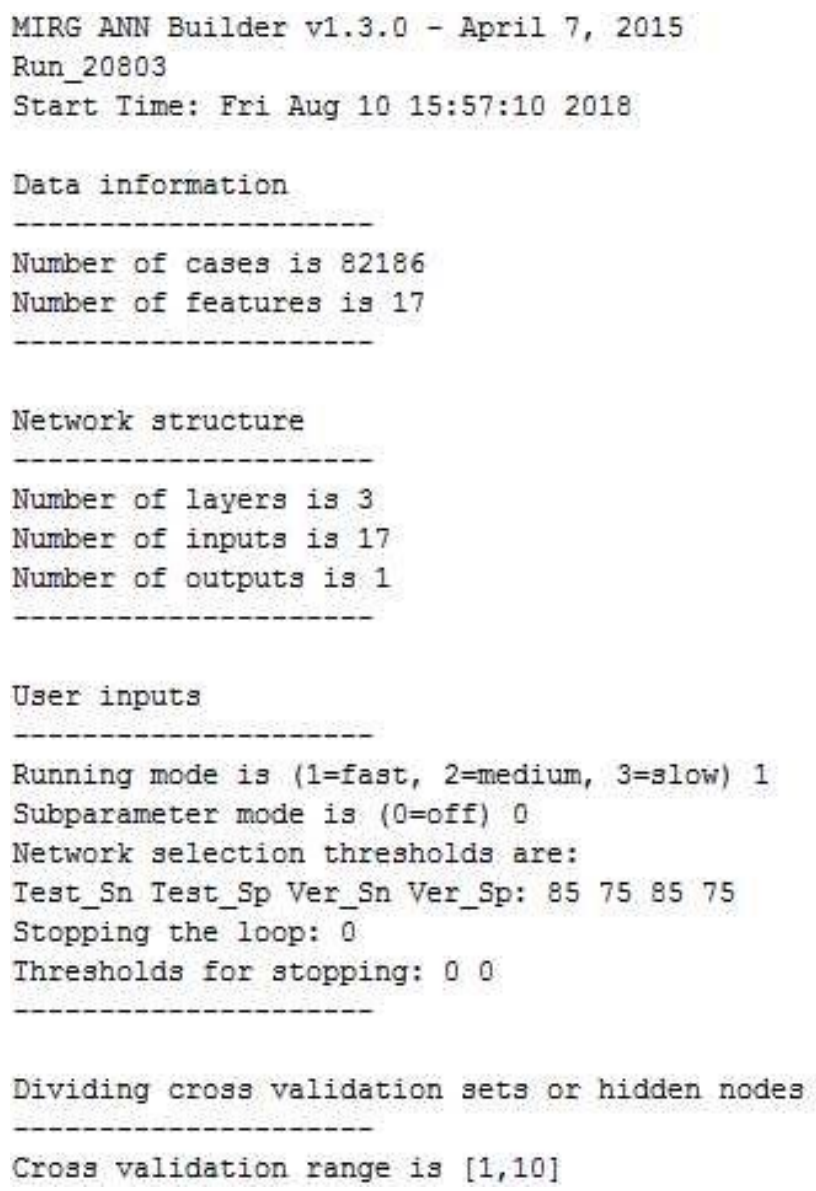

\section{Figure 3.5. Parameters for the BORN_Nulliparous dataset}

4. After balancing the classes there were 102,187 cases in the BORN dataset and 46,867 cases in the PRAMS dataset. From this, 20,001 cases from BORN, and 10,001 cases from PRAMS were put aside for the final testing stage of the validation set. Therefore, the BORN dataset had around 82,186 cases to be trained and the PRAMS dataset had around 36,866 cases to be trained with the ANN model.

5. The threshold to save the networks was chosen to be $60-85 \%$ for sensitivity and $70-$ $75 \%$ for specificity; these were the tested highest thresholds which would save the networks in the result files. 
6. The choice to optimize sensitivity over specificity was selected when selecting the best networks and the performance metrics from the results were saved in a separate CSV file (classifier_stats_final.csv).

7. The ANN Builder will create 10 networks which optimize the sensitivity metric

8. This final validation set is separate from the training, testing and verification sets created from 5-by-2 cross validation. The validation set consisted of 5,000 cases (4605 term and 395 preterm) for PRAMS_Parous and PRAMS_Nulliparous and 10,000 (9210 term and 790 preterm) cases for BORN_Parous and BORN_Nulliparous. This validation set had the labels removed before inputting the data into the ANN model for final classification of the output labels. These cases were aggregated by randomly sorting rows in Excel and selecting 790 or 395 preterm cases.

9. The performance metrics were calculated (sensitivity and specificity) for these four datasets and are described in detail in the next section

Execution of the ANN Builder software was used with the following parameters: FAST mode (tests around $0.5 \%$ of all possible combinations, excluding the number of hidden nodes), 3 hidden layers and the reported above network thresholds (see Step 5).

Another script was created: The Performance Measures Calculation Tool [13] for outputting the performance metrics of the best network. These metrics include the Positive Predictive Value (PPV), Negative Predictive Value (NPV), Accuracy (ACC), Matthews Correlation Coefficient (MCC), Receiver Operating Curve (ROC), F1-Score and Area Under the Curve (AUC). These statistical metrics were saved in the classifier_stats_final.csv.

The ANN Model Selection Tool [13] is a script which was used to select the best performing network based on the highest sensitivity. This selection script is applied to 10 result files (after 5- 
by-2 cross validation) and sorts through to find the final network with the highest sensitivity, within the verification and test sets. This final network was tested against the validation set (which is separate from the three datasets created from 5-by-2 Cross Validation and consists of 10,000 BORN or 5,000 PRAMS cases). More details regarding the network parameters can be found in Appendix C. 


\section{Chapter: Results and Discussion}

This chapter outlines the results of this thesis using data preparation methods and machine learning classifiers. The results stem from the C5.0 DT Classifier for feature reduction and the Artificial Neural Network Classifier for model evaluation.

Table 4.1. Results for the development and evaluation of the predictive tool

\begin{tabular}{|l|l|}
\hline \multicolumn{1}{|c|}{ Steps } & \multicolumn{1}{|c|}{ Results } \\
\hline Step 1: Data Visualization & Bar charts and plots showing the missing values within the data \\
\hline Step 2: Eliminated cases and features & Removed cases with greater than 50\% missing values and no \\
outcome label
\end{tabular}




\subsection{Step 1: Data Visualization}

The bar chart in Figure 4.1 compares a variety of clinical features: OTH_Term (Pregnancy history: other terminations?), Plural (Plurality births), Preghx (Pregnancy history: calculated from calculated from Previous Live Birth, Previous Low Birth Weight birth, and Previous Preterm birth), Prev_Lb (Previous live births) plotted against the Outcome feature (1 for a preterm outcome and -1 for a term outcome).

The small average difference between the Parous features reveals that there might not be a significant difference between these Parous clinical features and a preterm or term outcome. Therefore, more advanced algorithms are needed such as the C5.0 DT classifier to determine features of importance. However, the benefit of using Tableau is that it provides a visual basis to have conversations about features with our clinical partner, instead of looking at raw data from Excel. 


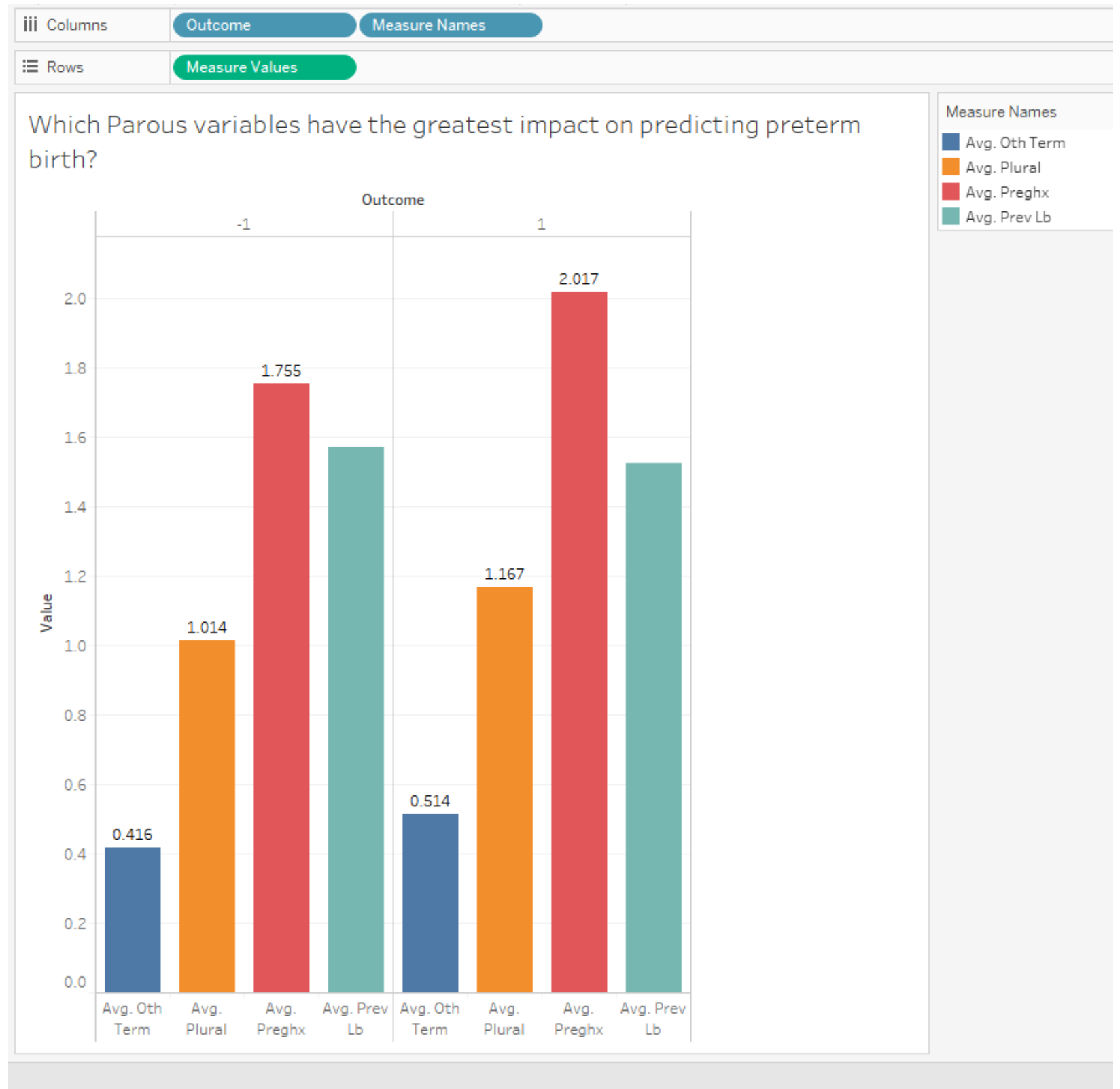

Figure 4.1. Bar Chart in Tableau comparing Parous_PRAMS features

Missing values were quite prevalent within both the BORN and PRAMS dataset. The below Missingness Maps (Figure 4.2 \& 4.3) were created to quickly see the presence of missing values within the clinical features using the Amelia package in $\mathrm{R}$ [92]. The $\mathrm{x}$-axis lists the features from the BORN or PRAMS dataset and the y-axis is the CASE ID. Comparing the same number of cases (50) the BORN dataset seems to have more missing values present than the PRAMS 
dataset, although this could be dependent on the specific features compared. Using more complex algorithms such as missForest are necessary when analyzing the missing values in detail. However, the benefit of using these maps is that it gives the user a quick insight into the general number of missing values present.

\section{Missingness Map}

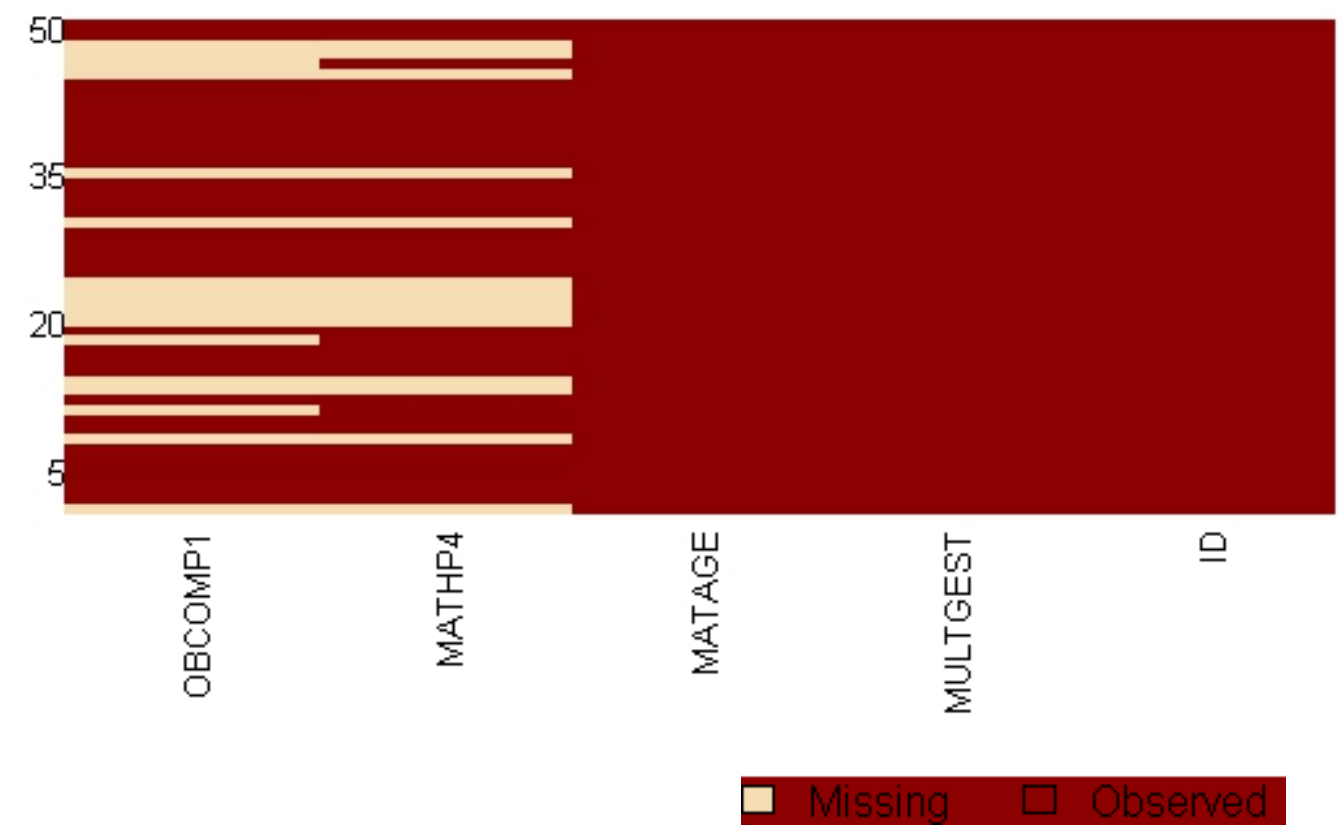

Figure 4.2. Missingness Map for the BORN_Nulliparous features 


\section{Missingness Map}

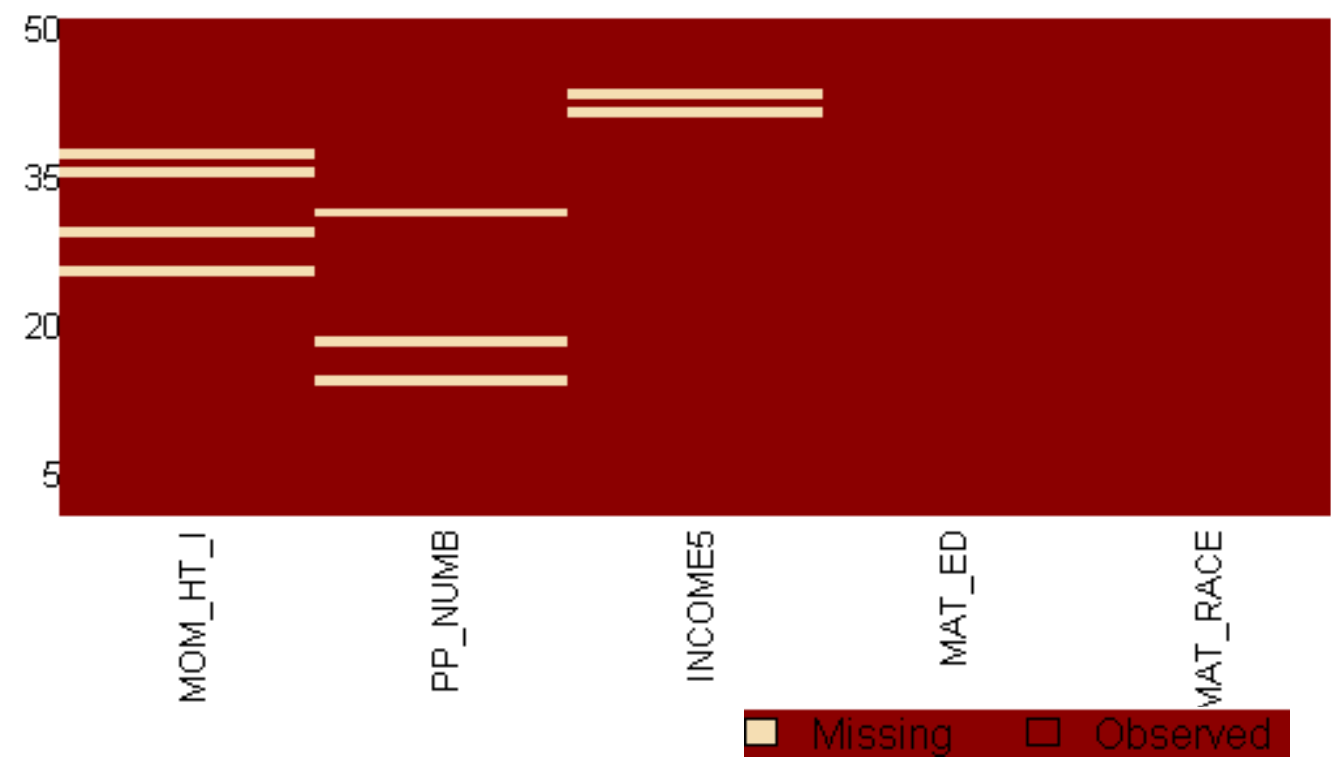

Figure 4.3 Missingness Map for the PRAMS_Nulliparous features

\subsection{Step 2: Eliminating Cases and Features}

Preliminary data preparation was done in Excel to remove features with greater than $50 \%$ missing values and missing OUTCOME labels, the final results are displayed in Table 4.2.

Table 4.2. Number of features prior to and after feature and case elimination

\begin{tabular}{|l|lc|lc|}
\hline & \multicolumn{2}{|l|}{ Before Feature and Case Elimination } & \multicolumn{2}{l|}{ After Feature and Case Elimination } \\
\hline PRAMS & \# Features: 372 & \# Cases: 109,319 & \# Features: 81 & \# Cases: 109,076 \\
\hline BORN & \# Features: 226 & \# Cases: 679,697 & \# Features: 200 & \# Cases: 669,134 \\
\hline
\end{tabular}




\subsection{Step 3: Choosing features with greater than $50 \%$ importance using the C5.0 DT classifier}

As previously stated, the C5.0 DT classifier has the capability to deal with missing values. This led to investigating whether one should fill in missing values before or after feature reduction. Although the sensitivity results were initially higher during training with the imputed values, (see Table 4.3), the final results show that when testing the classifier, the classifier performed generally worse. Therefore, it was decided to determine the features of importance prior to imputing the missing values, to reduce possible overfitting of the results. 
Table 4.3. Comparison of the two methodologies

\begin{tabular}{|c|c|c|}
\hline PRAMS Nulliparous Dataset & Option 1 & Option 2 \\
\hline Training Set (Sensitivity) & $79 \pm 0 \%$ & $76 \pm 0 \%$ \\
\hline Test Set (Sensitivity) & $74 \pm 1 \%$ & $74 \pm 1 \%$ \\
\hline PRAMS Parous Dataset & Option 1 & Option 2 \\
\hline Training Set (Sensitivity) & $79 \pm 1 \%$ & $77 \pm 0 \%$ \\
\hline Test Set (Sensitivity) & $72 \pm 1 \%$ & $74 \pm 0 \%$ \\
\hline BORN Parous Dataset & Option 1 & Option 2 \\
\hline Training Set (Sensitivity) & $88 \pm 0 \%$ & $87 \pm 0 \%$ \\
\hline Test Set (Sensitivity) & $82 \pm 0 \%$ & $87 \pm 0 \%$ \\
\hline BORN Nulliparous Dataset & Option 1 & Option 2 \\
\hline Training Set (Sensitivity) & $88 \pm 0 \%$ & $87 \pm 2 \%$ \\
\hline Test Set (Sensitivity) & $82 \pm 0 \%$ & $87 \pm 0 \%$ \\
\hline
\end{tabular}


The $50 \%$ threshold was determined experimentally by testing different feature subset sizes using the C5.0 DT classifier. It was found that including features with greater than $30 \%$ feature importance, sometimes resulted in slightly lower classification performance and greater computational time (see Table 4.4. \& 4.6.). Another test included features with greater than $65 \%$ importance (see Table $4.5 \&$ 4.7.). This resulted in a slight increase in accuracy, but this threshold resulted in the removal of clinical features deemed important by our clinician supervisor for predicting preterm birth, such as the feature, SGA_10 (small for gestation age) in the PRAMS dataset and INTBF (intention to breastfeed) in the BORN dataset. Therefore, to maintain a good balance, between including key clinical features and high accuracy, a feature importance threshold of $\geq 50 \%$ was selected.

Table 4.4. Increased feature size to include $\geq 30 \%$ feature importance (BORN)

\begin{tabular}{|c|r|r|c|}
\hline Performance Metric & \multicolumn{1}{|c|}{ Train } & \multicolumn{1}{c|}{ Test } & Standard \\
& & & Deviation \\
\hline Accuracy: & 0.85 & 0.84 & \pm 0.00 \\
\hline Sensitivity/Recall: & 0.91 & 0.90 & \pm 0.01 \\
\hline Specificity: & 0.78 & 0.78 & \pm 0.01 \\
\hline
\end{tabular}


Table 4.5. Reduced feature size to include $\geq 65 \%$ feature importance (BORN)

\begin{tabular}{|l|r|r|c|}
\hline Performance Metric & \multicolumn{1}{|c|}{ Train } & Test & Standard \\
& & & Deviation \\
\hline Accuracy: & 0.84 & 0.84 & \pm 0.00 \\
\hline Sensitivity/Recall: & 0.91 & 0.90 & \pm 0.01 \\
\hline Specificity: & 0.77 & 0.77 & \pm 0.01 \\
\hline
\end{tabular}

Table 4.6. Increased feature size to include $\geq 30 \%$ feature importance (PRAMS)

\begin{tabular}{|c|c|c|c|}
\hline Performance Metric & Train & Test & Standard \\
& & & Deviation \\
\hline Accuracy: & 0.84 & 0.84 & \pm 0.01 \\
\hline Sensitivity/Recall: & 0.91 & 0.91 & \pm 0.01 \\
\hline Specificity: & 0.77 & 0.76 & \pm 0.03 \\
\hline
\end{tabular}

Table 4.7. Reduced feature size to include $\geq 65 \%$ feature importance (PRAMS)

\begin{tabular}{|c|c|c|c|}
\hline Performance Metric & Train & Test & Standard Deviation \\
\hline Accuracy: & 0.85 & 0.85 & \pm 0.00 \\
\hline Sensitivity/Recall: & 0.92 & 0.92 & \pm 0.01 \\
\hline Specificity: & 0.77 & 0.76 & \pm 0.01 \\
\hline
\end{tabular}

The C5.0 DT classifier outputs the percentage attributes of the features of importance without any preprocessing methods being applied to the data. It was important to not manipulate the data 
so that, when the ANN classifier encounters new test data, this does not result in the classifier overfitting the data.

The C5.0 DT classifier outputs ten trees and displays the feature importance for each tree. To maintain a good balance between the importance and the quantity of features chosen for each dataset, only features which had $\geq 50 \%$ feature importance were kept. Although most features had the same percentage attribute across all ten of the trees such as the features: PLURAL and MULTGEST (100\%), there were some features that differed among the ten trees which were close to the $50 \%$ threshold. In these cases, features were averaged across the ten trees and the feature with the highest percentage average was chosen.

There were some obvious features that were selected by the classifier such as GENDER (baby's sex) and PPRETERM (number of previous preterm babies) in the BORN dataset and MAT_AGE_NAPHSIS (maternal age grouped) and MAT_RACE (maternal race) in the PRAMS dataset. These features have been documented to be increased risk factors for a preterm birth outcome [30]. Some non-obvious features that were selected included PRENCLAS (number of prenatal classes in weeks) and INTBF (intention to breastfeed) in the BORN dataset and MAT_ED (maternal education) and PP_NUMB (\# sources of payment for prenatal classes) in the PRAMS dataset, these non-obvious features might allude to a certain socioeconomic status, leading to an increased/decreased risk of preterm birth.

Once these features were reduced from over 300 features in PRAMS and over 200 features in BORN (Table 4.8), this significantly decreased processing time and more importantly increased the sensitivity results; as the subset of features chosen, were directly important to predicting a preterm birth outcome. 
Table 4.8. Feature reduction results after applying the C5.0 DT classifier to the BORN and PRAMS datasets

\begin{tabular}{|l|l|l|}
\hline Datasets & Before Feature Reduction & After Feature Reduction \\
\hline BORN_Parous & \# Features: 200 & \# Features: 20 \\
\hline BORN_Nulliparous & \# Features: 180 & \# Features: 17 \\
\hline PRAMS_Parous & \# Features: 81 & \# Features: 22 \\
\hline PRAMS_Nulliparous & \# Features: 75 & \# Features: 20 \\
\hline
\end{tabular}

The following Tables 4.9-4.12 display the reduced features subsets (threshold of $\geq 50 \%$ ) output by the

C5.0 DT classifier for BORN and PRAMS

Table 4.9: 20 Features: Parous BORN

\begin{tabular}{|l|l|}
\hline $100 \%$ MULTGEST: multiple gestation & $95 \%$ PRENCLAS: Prenatal class \\
\hline elsewhere - Other syndromes & $80 \%$ GENDER: Baby's sex \\
\hline $82 \%$ OBCOMP12: Preterm premature rupture of & $74 \%$ OBCOMP1: Obstetrical complications \\
membranes (PPROM) & (Eclampsia) \\
\hline $76 \%$ PREVCS: Previous cesarean Section & $74 \%$ OBCOMP9: Pre-eclampsia \\
\hline $74 \%$ OBCOMP8: Obstetrical complications & $70 \%$ OBCOMP7: Obstetrical complications \\
(Placental abruption) & (Placenta previa) \\
\hline $71 \%$ OBCOMP4: IUGR/SGA (Small for & $69 \%$ OBCOMP10: Premature rupture of \\
gestational age) & membranes (PROM) \\
\hline $69 \%$ PPRETERM: Number of previous preterm & $64 \%$ OBCOMP3: Hypertension (gestational or \\
babies & transient) \\
\hline $64 \%$ MATAGE: Mother's age (years) & $61 \%$ MATHP27: Other Maternal Health Problem \\
\hline
\end{tabular}




\begin{tabular}{|l|l|}
\hline $\begin{array}{l}\text { 62\% MATHP4: Maternal health problem } \\
\text { (Diabetes insulin) }\end{array}$ & $60 \%$ MATHP3: Chronic hypertension \\
\hline $\begin{array}{l}\text { 55\% OBCOMP5: Obstetrical complications } \\
\text { (Large for Gestational Age) }\end{array}$ & $50 \%$ INTBF: Intention to Breastfeed \\
\hline
\end{tabular}

Table 4.10: 17 Features: Nulliparous BORN

\begin{tabular}{|l|l|}
\hline $100 \%$ MULTGEST & $80 \%$ GENDER \\
\hline $80 \%$ PRENCLAS & $79 \%$ OBCOMP12 \\
\hline $77 \%$ CONGAN55 & $76 \%$ MATAGE \\
\hline $74 \%$ OBCOMP1 & $74 \%$ OBCOMP8 \\
\hline $74 \%$ OBCOMP9 & $70 \%$ OBCOMP4 \\
\cline { 1 - 2 } $69 \%$ OBCOMP7 & $68 \%$ OBCOMP10 \\
\cline { 1 - 2 } $65 \%$ MATHP27 & $62 \%$ OBCOMP3 \\
\cline { 1 - 2 } $62 \%$ MATHP3 & $57 \%$ MATHP4 \\
\cline { 1 - 2 } $58 \%$ OBCOMP5: LGA (large for gestational age) & \multicolumn{1}{|l}{} \\
\cline { 1 - 2 } &
\end{tabular}

Table 4.11: 22 Features: Parous PRAMS

\begin{tabular}{|l|l|}
\hline $100 \%$ PLURAL: plurality & $100 \%$ MM_PROM: rupture membrane? \\
\hline prenatal care visits grouped & $93 \%$ MM_HBP: hypertension? \\
\hline $87 \%$ PREGHX: pregnancy history & $84 \%$ MOMLBS: maternal weight gain \\
\hline $84 \%$ SGA_2SD: small for gestational age & $83 \%$ LGA: large for gestational age based on \\
based on 2SD from mean & 90 th percentile \\
\hline
\end{tabular}




\begin{tabular}{|l|l|}
\hline $\begin{array}{l}83 \% \text { PNC_MTH: month of first prenatal care } \\
\text { visit }\end{array}$ & $\begin{array}{l}79 \% \text { DEFECT: defect present? (this can be } \\
\text { detected during an antenatal ultrasound) }\end{array}$ \\
\hline $82 \%$ MM_FEVER: fever (mother)? & $76 \%$ MAT_RACE: maternal race \\
\hline $\begin{array}{l}77 \% \text { OTH_TERM: pregnancy history (other } \\
\text { terminations?) }\end{array}$ & $72 \%$ MOM_HT_I: mom total height (inches) \\
\hline $73 \%$ BC_YRLLB: years since last live birth & $67 \%$ PNC_WKS: weeks ${ }^{\text {st }}$ prenatal care \\
visit \\
\hline $71 \%$ INCOME5: 12 months before, total \\
income & $64 \%$ MM_NOMD: no medical risk factors? \\
\hline $\begin{array}{l}66 \% \text { PRE_LB_NAPHSIS: number of } \\
\text { previous live births grouped }\end{array}$ & $61 \%$ SGA_10: small for gestational age \\
\hline $54 \%$ PP_NUMB: \# sources of payment for \\
prenatal care
\end{tabular}

Table 4.12: 19 Features: Nulliparous PRAMS

\begin{tabular}{|l|l|}
\hline $100 \%$ PLURAL & $100 \%$ MM_PROM \\
\hline $100 \%$ MACROSOMIA: Macrosomia: & $100 \%$ PNC_VST_NAPHSIS \\
\hline 4500 gram birth weight & $88 \%$ LGA \\
\hline $80 \%$ MM_HBP & $85 \%$ SGA_2SD \\
\hline $81 \%$ MOMLBS & $81 \%$ MM_NOMD: \\
\hline $79 \%$ PNC_MTH & $79 \%$ DEFECT \\
\hline
\end{tabular}




\begin{tabular}{|l|l|}
\hline $78 \%$ MM_FEVER & $78 \%$ MAT_RACE \\
\hline $77 \%$ MAT_ED: maternal education & $78 \%$ MOM_HT_I \\
\hline $\begin{array}{ll}\text { 67\% PP_NUMB: \# sources of payment for } \\
\text { prenatal care }\end{array}$ & $\begin{array}{l}56 \% \text { MAT_AGE_NAPHSIS: maternal age } \\
\text { grouped }\end{array}$ \\
\cline { 1 - 2 } $51 \%$ SURE_WKS: weeks when sure pregnant & \multicolumn{2}{|l}{} \\
\cline { 1 - 2 } &
\end{tabular}

There are several similar features chosen by the C5.0 DT classifier between the PRAMS and BORN datasets (see Figure 4.4.) Despite the differences, which include how the data has been obtained, the features which have been measured, and the location of the clinical datasets, this classifier has determined key features of importance when predicting a premature birth. These features are important to record if one wants to build their own preterm birth dataset specific to a clinical site. By inputting these features into the neural network, there is a high probability of improved sensitivity results. Some of these features include: maternal age, if the mother has high blood pressure, presence of previous premature births etc.... There are also similar features chosen between Ong's research work [24] (see Table 4.13-4.16). These features are of importance when predicting preterm birth, as they tend to appear across various research reports on preterm birth. 


\begin{tabular}{|c|c|}
\hline BORN & PRAMS \\
\hline MULTGEST: multiple gestation & PLURAL: plurality \\
\hline $\begin{array}{l}\text { OBCOMP10 \& OBCOMP12: premature } \\
\text { rupture of membranes (PROM) \& Preterm } \\
\text { premature rupture of membranes (PPOM) }\end{array}$ & MM_PROM: ruptured membranes? \\
\hline OBCOMP4: Small for gestational age & $\begin{array}{l}\text { SGA_2SD \& SGA_10: Small for gestational } \\
\text { age based on } 2 \text { standard deviation from the } \\
\text { mean \& Small for gestational age based on } \\
\qquad 10^{\text {th }} \text { percentile }\end{array}$ \\
\hline OBCOMP5: Large for gestational age & $\begin{array}{l}\text { LGA: Large for gestational age based on the } \\
\qquad 90^{\text {th }} \text { percentile }\end{array}$ \\
\hline MATAGE: Mother's age in years & $\begin{array}{c}\text { MAT_AGE_NAPHSIS: Maternal age } \\
\text { grouped }\end{array}$ \\
\hline $\begin{array}{c}\text { PPRETERM: Number of previous preterm } \\
\text { babies }\end{array}$ & $\begin{array}{l}\text { PREGHX: Pregnancy history (calculated } \\
\text { from Previous Live Birth, Previous Low Birth } \\
\text { Weight birth, and Previous Preterm birth) }\end{array}$ \\
\hline $\begin{array}{l}\text { OBCOMP3 \& MATHP3 (Hypertension } \\
\text { gestational or transien \& Chronic } \\
\text { hypertension }\end{array}$ & MM_HBP: Hypertension? \\
\hline
\end{tabular}

Figure 4.4. List of abbreviations used for highly ranked features which occurred in both the BORN and PRAMS data sets, used in this study to assess risk of preterm birth 
Table 4.13: Similar features chosen in current and earlier research work: Parous_BORN

\begin{tabular}{|c|c|}
\hline $100 \%$ MULTGEST & $95 \%$ PRENCLAS \\
\hline $95 \%$ CONGAN55 & $80 \%$ GENDER \\
\hline $82 \%$ OBCOMP12 & $74 \%$ OBCOMP1 \\
\hline $76 \%$ PREVCS & $74 \%$ OBCOMP9 \\
\hline $74 \%$ OBCOMP8 & $70 \%$ OBCOMP7 \\
\hline $71 \%$ OBCOMP4 & $69 \%$ OBCOMP10 \\
\hline $69 \%$ PPRETERM & $64 \%$ OBCOMP3 \\
\hline $64 \%$ MATAGE & $61 \%$ MATHP27 \\
\hline $62 \%$ MATHP4 & $60 \%$ MATHP3 \\
\hline $55 \%$ OBCOMP5 & $50 \%$ INTBF \\
\hline
\end{tabular}

These features were also present in past work

Table 4.14: Similar features chosen in current and earlier research work: Nulliparous_BORN

\begin{tabular}{|c|c|}
\hline $100 \%$ MULTGEST & $80 \%$ GENDER \\
\hline $80 \%$ PRENCLAS & $79 \%$ OBCOMP12 \\
\hline $77 \%$ CONGAN55 & $76 \%$ MATAGE \\
\hline $74 \%$ OBCOMP1 & $74 \%$ OBCOMP8 \\
\hline $74 \%$ OBCOMP9 & $70 \%$ OBCOMP4 \\
\hline $69 \%$ OBCOMP7 & $68 \%$ OBCOMP10 \\
\hline $65 \%$ MATHP27 & $62 \%$ OBCOMP3 \\
\hline $62 \%$ MATHP3 & $57 \%$ MATHP4 \\
\hline $58 \%$ OBCOMP5 & \\
\cline { 1 - 2 } & \\
\cline { 1 - 2 } &
\end{tabular}

These features were also present in past work 
Table 4.15: Similar features chosen in current and earlier research work: Parous_PRAMS

\begin{tabular}{|c|c|}
\hline 100\% PLURAL: plurality & $100 \%$ MM_PROM: rupture membrane? \\
\hline $\begin{array}{l}\text { 99\% PNC_VST_NAPHSIS: number of } \\
\text { prenatal care visits grouped }\end{array}$ & 93\% MM_HBP: hypertension? \\
\hline 87\% PREGHX: pregnancy history & 84\% MOMLBS: maternal weight gain \\
\hline $\begin{array}{l}\text { 84\% SGA_2SD: small for gestational age } \\
\text { based on 2SD from mean }\end{array}$ & $\begin{array}{l}\text { 83\% LGA: large for gestational age based on } \\
\text { 90th percentile }\end{array}$ \\
\hline $\begin{array}{l}\text { 83\% PNC_MTH: month of first prenatal care } \\
\text { visit }\end{array}$ & $\begin{array}{l}\text { 79\% DEFECT: defect present? (this can be } \\
\text { detected during an antenatal ultrasound) }\end{array}$ \\
\hline 82\% MM_FEVER: fever (mother)? & 76\% MAT_RACE: maternal race \\
\hline $\begin{array}{l}\text { 77\% OTH_TERM: pregnancy history (other } \\
\text { terminations?) }\end{array}$ & 72\% MOM_HT_I: mom total height (inches) \\
\hline 73\% BC_YRLLB: years since last live birth & $\begin{array}{l}\text { 67\% PNC_WKS: weeks } 1^{\text {st }} \text { prenatal care } \\
\text { visit }\end{array}$ \\
\hline $\begin{array}{l}\text { 71\% INCOME5: } 12 \text { months before, total } \\
\text { income }\end{array}$ & 64\% MM_NOMD: no medical risk factors? \\
\hline $\begin{array}{l}66 \% \text { PRE_LB_NAPHSIS: number of } \\
\text { previous live births grouped }\end{array}$ & $\begin{array}{l}\text { 61\% SGA_10: small for gestational age } \\
\text { based on 10th percentile }\end{array}$ \\
\hline $\begin{array}{l}54 \% \text { PP_NUMB: \# sources of payment for } \\
\text { prenatal care }\end{array}$ & 51\% MAT_ED: maternal education \\
\hline
\end{tabular}

These features were also present in past work 
Table 4.16: Similar features chosen in current and earlier research work: Nulliparous_PRAMS

\begin{tabular}{|c|c|}
\hline $100 \%$ PLURAL & $100 \%$ MM_PROM \\
\hline $\begin{array}{l}\text { 100\% MACROSOMIA: Macrosomia: } \\
\geq 4500 \text { gram birth weight }\end{array}$ & $100 \%$ PNC_VST_NAPHSIS \\
\hline 90\% MM_HBP & $88 \%$ LGA \\
\hline $87 \%$ MOMLBS & $85 \%$ SGA_2SD \\
\hline $81 \%$ INCOME5 & 81\% MM_NOMD: \\
\hline 79\% PNC_MTH & $79 \%$ DEFECT \\
\hline 78\% MM_FEVER & 78\% MAT_RACE \\
\hline 77\% MAT_ED: maternal education & 78\% MOM_HT_I \\
\hline $\begin{array}{l}\text { 67\% PP_NUMB: \# sources of payment for } \\
\text { prenatal care }\end{array}$ & $\begin{array}{l}\text { 56\% MAT_AGE_NAPHSIS: maternal age } \\
\text { grouped }\end{array}$ \\
\hline 51\% SURE_WKS: weeks when sure pregnant & \\
\hline
\end{tabular}

These features were also present in past work

\subsection{Step 4: Balancing the Classes}

When applying this function, this greatly reduced the number of cases in the training dataset (See Table 4.17.) and improved the computational training time of the neural network. For instance, in work done by Ong [24], the BORN_Parous and BORN_Nulliparous datasets took around 634 hours and 186 hours respectively to train, whereas the PRAMS_Parous and PRAMS_Nulliparous took 73 and 36 hours respectively to train. In this work, with the reduction of the dataset, the BORN_Parous and BORN_Nulliparous datasets took around 10 and 9 hours respectively to train, whereas the PRAMS_Parous and PRAMS_Nulliparous took around 4 hours each to train. The hardware specifications of the computers on which the models were trained on were similar. 
All simulations in this current work were run on BME-12 lab computers: Intel Core i5 760 (2.80GHz) processor, 8GB RAM, Windows 7 64bit. Where Ong [24], ran her models on the BME-12 and BME-14 lab computer: Intel Core i7-3770 (3.4GHz) processor, 8 GB RAM, Windows 7 64bit.

Table 4.17. Case reduction results after applying package in $R$ (ubBalance) to the $B O R N$ and PRAMS datasets

\begin{tabular}{|l|l|l|}
\hline & Before Class Balance & After Class Balance \\
\hline BORN & \# Cases: 679,697 & \# Cases: 102,187 \\
\hline PRAMS & \# Cases: 109,079 & \# Cases: 46,867 \\
\hline
\end{tabular}

\subsection{Step 5: Input missing values}

As stated previously, missForest uses random forest classifiers. These classifiers do not need to perform 5-by-2 cross validation to create a test set such as with the DT classifier or with the ANN classifier because random forests contain internal test sets to estimate the error. During the random forest run around $1 / 3$ of cases are not used during training and are instead used as a test set. The out of bag (OOB) imputation error supplied two values for the categorical and nominal features and the results are displayed in Table 4.18. The proportion of falsely classified (PFC) cases represents the error for the categorical features and the normalized mean square error (NMSE) represents the error for the nominal features [48].

Table 4.18 OOB error estimate for Nulliparous_PRAMS dataset

NRMSE: 0.2353279

PFC: 0.3191449 


\subsection{Step 6: Normalizing the data}

Results from normalizing the data using the modified Z-score transformation are displayed in Figure 4.5., saved in a data file. The data is scaled between a range of -1 to 1 .

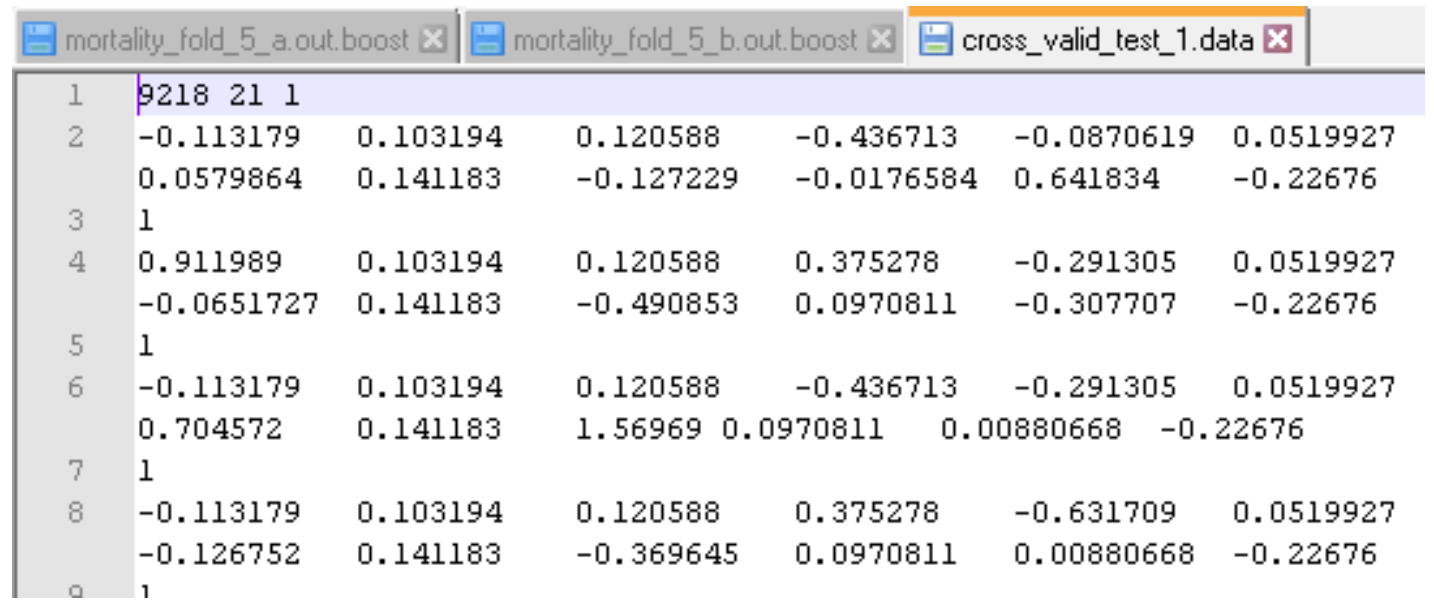

Figure 4.5. Results of data normalization

\subsection{Step 7: Divide into test, train and verification sets}

The results from applying the BASH file ./create_5_by_2.sh are displayed in the below Figure 4.6. Once these files have been created, then the training of the neural networks using the ANN builder [13] can commence.

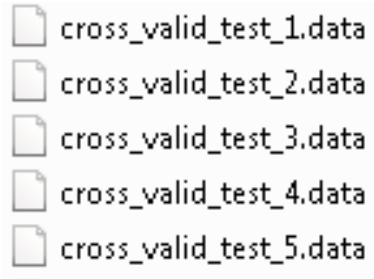

$\begin{array}{ll}\text { 23/02/2018 10:45 ... } & \text { DATA File } \\ \text { 23/02/2018 10:45 ... } & \text { DATA File } \\ \text { 23/02/2018 10:45 ... } & \text { DATA File } \\ 23 / 02 / 201810: 45 \ldots & \text { DATA File } \\ 23 / 02 / 201810: 45 \ldots & \text { DATA File }\end{array}$

Figure 4.6. Results of 5-by-2 Cross Validation (test set)

\subsection{Step 8: Execution of the ANN Builder}

After 5-by-2 cross validation was applied to create train, test and verification sets for the ANN, around 100 neural networks were saved in the network folders and the best neural network which optimized sensitivity was selected. An example of the performance metrics for the PRAMS_Parous classifier is displayed below in Table 4.19. This neural network was then tested 
on the validation set for final testing. The validation set for the BORN dataset consisted of 10,000 cases set aside for final testing. Similarly, the validation set for the PRAMS dataset consisted of 5,000 cases set aside for final testing, A division of the data is described in Figure 4.7. The OUTCOME feature for the validation sets was removed and unlabeled data was fed into the ANN, to determine the predictive performance of the classifier. Both of these validation sets had $7.9 \%$ prevalence to match the population of preterm birth in Ontario.

Table 4.19. Performance Metrics for the PRAMS_Parous classifier

\begin{tabular}{|l|r|r|}
\hline & \multicolumn{1}{|c|}{ Average } & \multicolumn{1}{c|}{$\begin{array}{c}\text { Standard } \\
\text { deviation }\end{array}$} \\
\hline Sensitivity & 0.703364 & 0.006915 \\
\hline Specificity & 0.734787 & 0.009838 \\
\hline PPV & 0.726267 & 0.005978 \\
\hline NPV & 0.712427 & 0.003266 \\
\hline ACC & 0.719076 & 0.003314 \\
\hline MCC & 0.438422 & 0.006682 \\
\hline F1score & 0.714586 & 0.003064 \\
\hline AUC & 0.788326 & 0.0041 \\
\hline
\end{tabular}



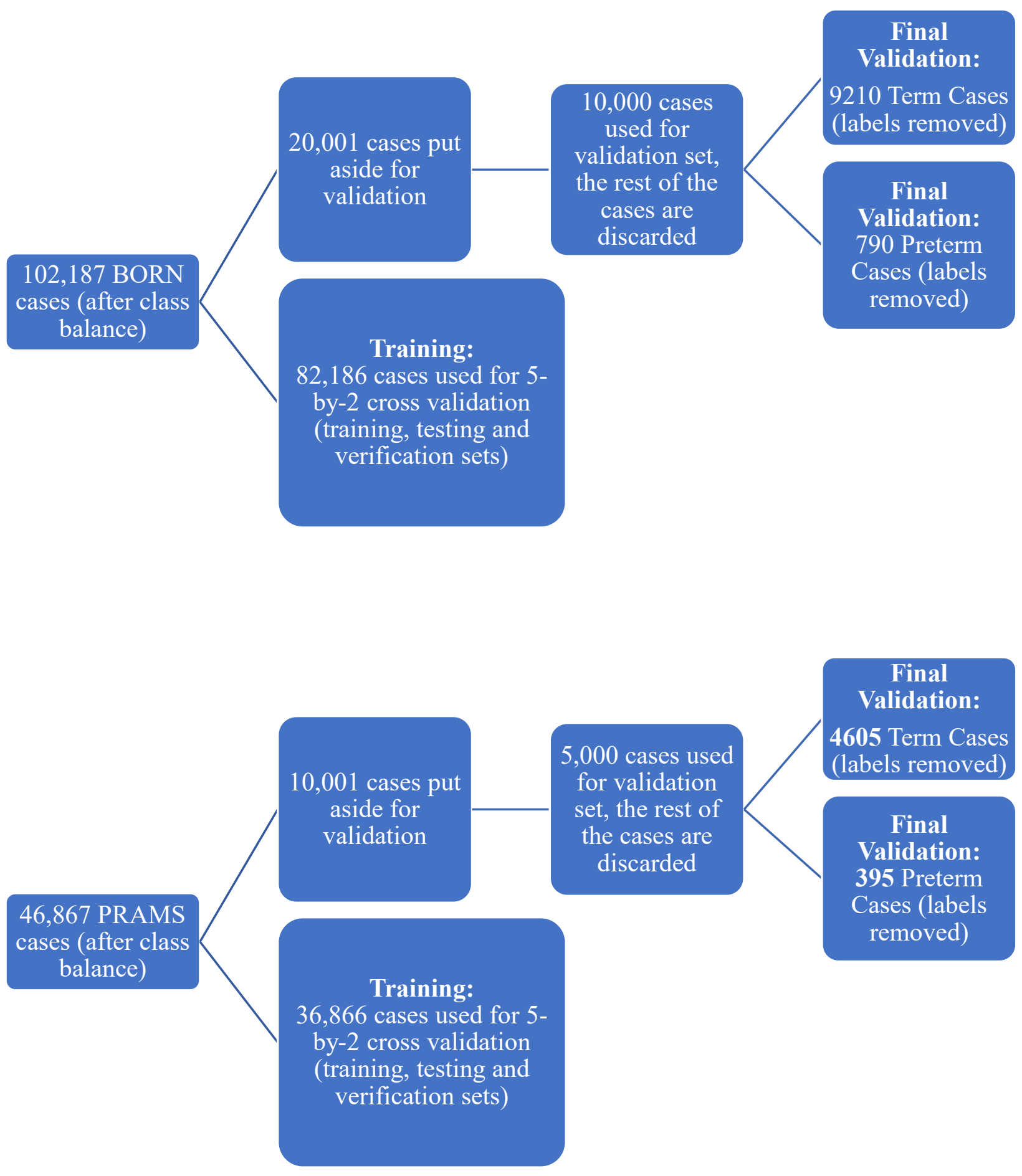

Figure 4.7 Division of the BORN and PRAMS dataset: training, testing, verification and validation data 
The confusion matrix was calculated for each of the four datasets which were tested against the final validation set (10,000 for BORN and 5,000 for PRAMS) the confusion matrix, sensitivity and specificity metrics are displayed below (Table 4.20-4.27). In an imbalanced dataset which is what the ANN is tested on with $7.9 \%$ prevalence, the accuracy metric is not the most effective measure for determining the performance of the classifier. Since there is such a small proportion of preterm cases, the classifier might not be able to distinguish between the preterm cases (low true positive rate) but could instead classify the term cases with a high accuracy (high true negative rate) leading to an overall high accuracy metric. A better metric to compare the classifier's performance is the AUC (area under the curve) which aggregates the classifier performance at a variety of thresholds. In addition, due to this class imbalance there are many term cases which could be classified as false positives (falsely classified as preterm), this contributes to the low precision value (1) reported in the below tables. Conversely, due to the high number of term cases, there are few preterm cases which could be classified as term, the focus of this research is to limit this occurrence and contributes to the high negative predictive value (2) reported in the below tables.

$$
\begin{aligned}
& P P V=\frac{T P}{T P+F P} \times 100(1) \\
& N P V=\frac{T N}{T N+F N} \times 100(2)
\end{aligned}
$$


Table 4.20. Confusion Matrix: Parous_BORN Verfication Results at 7.9\% Prevalence Unlabeled Data

\begin{tabular}{|l|l|l|}
\hline & Predicted Term & Predicted Preterm \\
\hline Actual & TN:6241 & FP: 2969 \\
\hline Actual & FN:85 & TP: 705 \\
\hline Preterm & & \\
\hline
\end{tabular}

Sensitivity $=89.2 \%$

Specificity $=67.8 \%$

Table 4.21. Performance Metrics: Parous_BORN Verfication Results at 7.9\% Prevalence Unlabeled Data

\begin{tabular}{|l|l|}
\hline Accuracy & 0.69 \\
\hline Sensitivity/Recall & 0.89 \\
\hline Specificity & 0.68 \\
\hline PPV/Precision & 0.19 \\
\hline NPV & 0.99 \\
\hline MCC & 0.32 \\
\hline
\end{tabular}


Table 4.22. Confusion Matrix: Nulliparous_BORN Verfication Results at 7.9\% Prevalence Unlabeled Data

\begin{tabular}{|l|l|l|}
\hline & Predicted Term & Predicted Preterm \\
\hline Actual & TN:6584 & FP: 2626 \\
\hline $\begin{array}{l}\text { Actual } \\
\text { Preterm }\end{array}$ & FN:87 & \\
\hline
\end{tabular}

Sensitivity $=89.0 \%$

Specificity $=71.5 \%$

Table 4.23. Performance Metrics Nulliparous_BORN Verfication Results at 7.9\% Prevalence Unlabeled Data

\begin{tabular}{|c|c|}
\hline Accuracy: & 0.73 \\
\hline Sensitivity/Recall: & 0.89 \\
\hline Specificity: & 0.72 \\
\hline PPV/Precision: & 0.21 \\
\hline NPV: & 0.99 \\
\hline F1 score: & 0.34 \\
\hline MCC: & 0.35 \\
\hline
\end{tabular}


Table 4.24. Confusion Matrix: Parous_PRAMS Verfication Results at 7.9\% Prevalence Unlabeled Data

\begin{tabular}{|l|l|l|}
\hline & Predicted Term & Predicted Preterm \\
\hline Actual & TN:3288 & FP: 1317 \\
\hline Actual & FN:63 & TP: 332 \\
\hline Preterm & & \\
\hline
\end{tabular}

Sensitivity $=84.1 \%$

Specificity $=71.4 \%$

Table 4.25. Performance Metrics: Parous_PRAMS Verfication Results at 7.9\% Prevalence Unseen Data

\begin{tabular}{|l|c|}
\hline Accuracy: & 0.73 \\
\hline Sensitivity/Recall: & 0.84 \\
\hline Specificity: & 0.71 \\
\hline PPV/Precision: & 0.21 \\
\hline NPV: & 0.99 \\
\hline F1 score: & \\
\hline MCC: & 0.34 \\
\hline
\end{tabular}


Table 4.26. Confusion Matrix: Nulliparous_PRAMS Verfication Results at 7.9\% Prevalence

\section{Unseen Data}

\begin{tabular}{|l|l|l|}
\hline & Predicted Term & Predicted Preterm \\
\hline Actual & TN:3501 & FP: 1104 \\
\hline $\begin{array}{l}\text { Actual } \\
\text { Preterm }\end{array}$ & FN:64 & \\
\hline
\end{tabular}

Sensitivity $=83.8 \%$

Specificity $=76.0 \%$

Table 4.27. Performance Metrics: Nulliparous_PRAMS Verfication Results at 7.9\% Prevalence Unseen Data

\begin{tabular}{|l|l|}
\hline Accuracy: & 0.77 \\
\hline Sensitivity/Recall: & 0.84 \\
\hline Specificity: & 0.76 \\
\hline PPV/Precision: & 0.23 \\
\hline NPV: & 0.98 \\
\hline F1 score: & 0.37 \\
\hline MCC: & 0.36 \\
\hline
\end{tabular}

The ROC Curves for each dataset (Figure $4.8-4.11$ ) are displayed below. The True Positive Rate (Sensitivity) is displayed on the y-axis and the False Positive Rate (1-Specificity) is displayed on the x-axis. Each point on the ROC curves represents the Sensitivity versus 1- 
Specificity at a specific threshold (e.g. 0,0.01...1). The AUC is a measure of how accurate the classifier predictions are in predicting preterm birth, all of the curves (Figure 4.2-4.5) tend to fall under the category of "Acceptable" $(>0.7)$ or "Excellent" $(>0.8)$ when measuring the effectiveness of these classifiers.

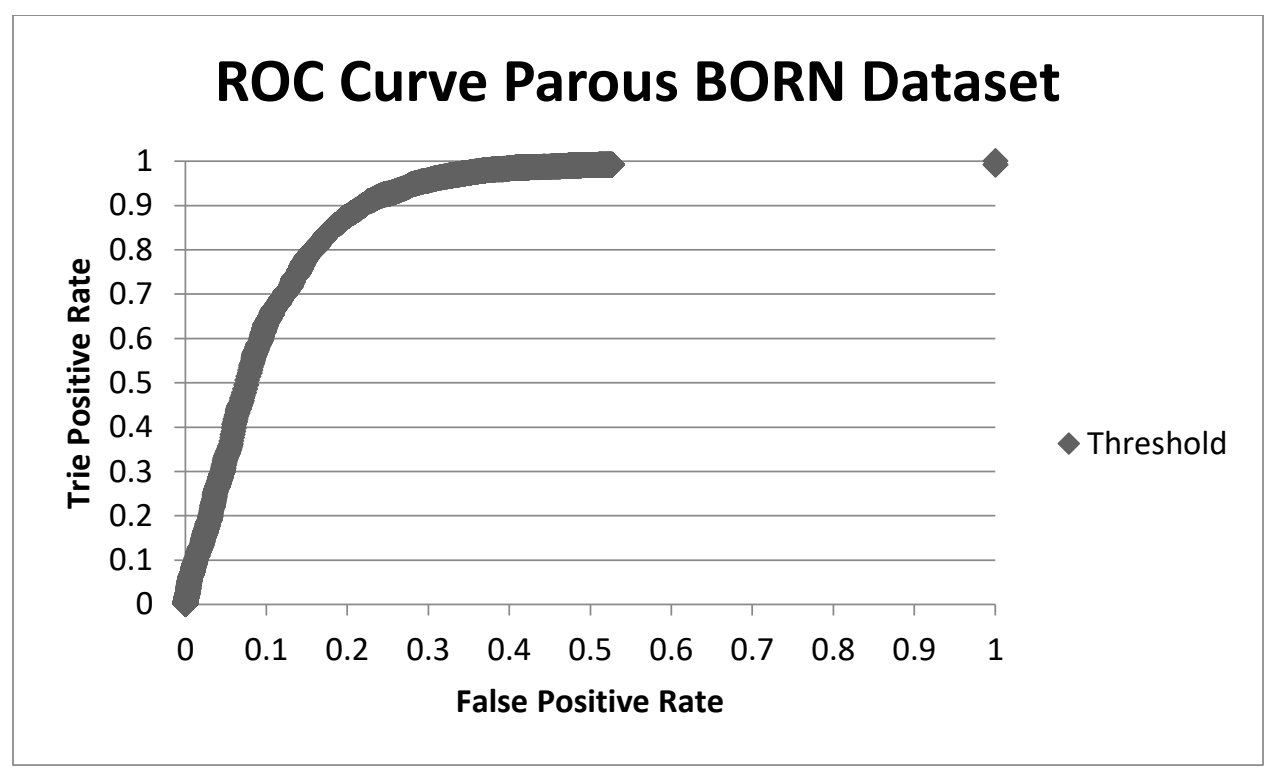

AUC: 0.894369

Figure 4.8. ROC Curve Performance for BORN Parous Dataset 


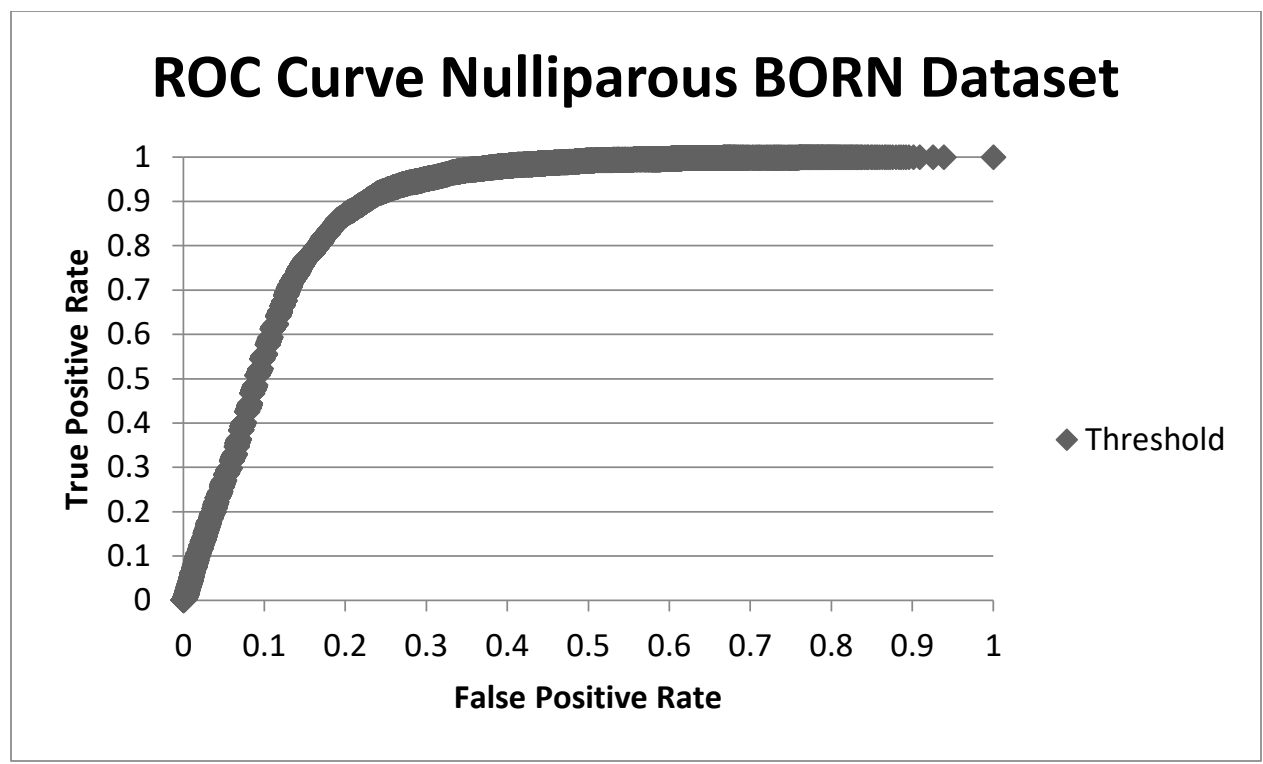

AUC: 0.888154

Figure 4.9. ROC Curve Performance for BORN Nulliparous Dataset

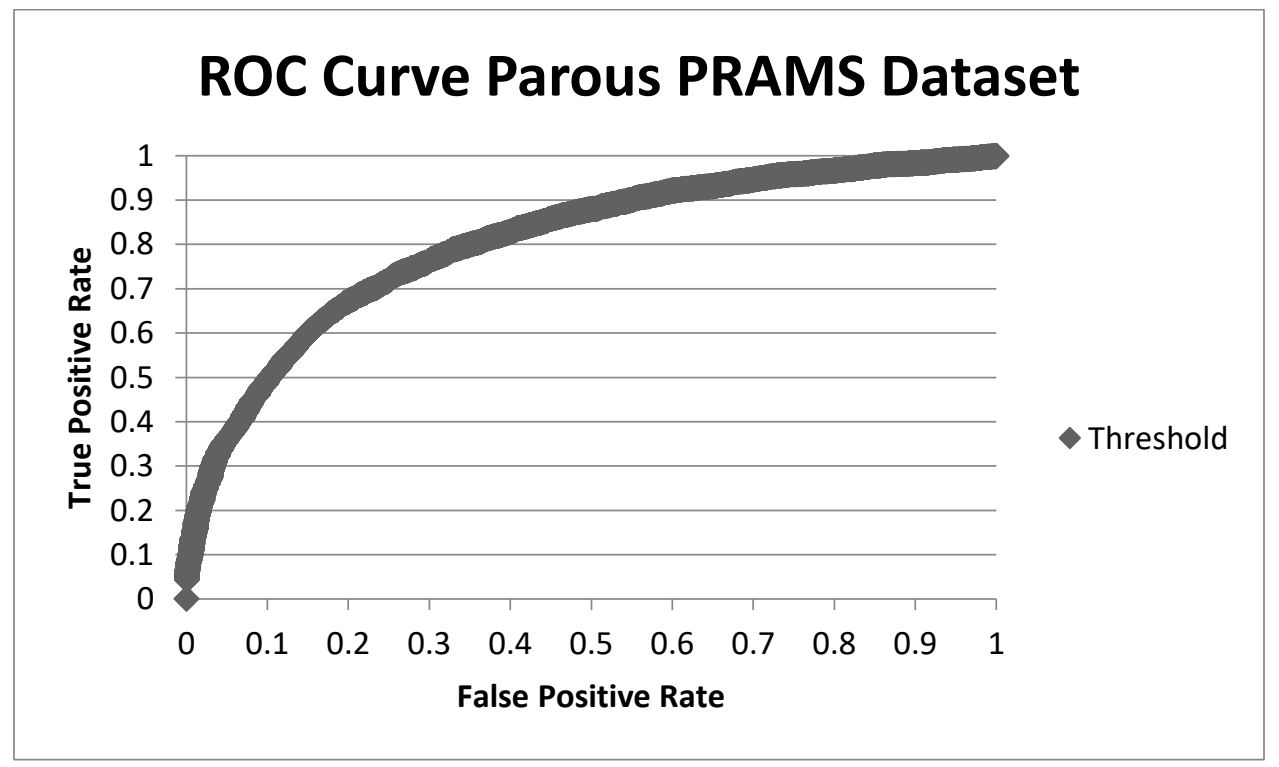

AUC: 0.805855

Figure 4.10. ROC Curve Performance for PRAMS Parous Dataset 


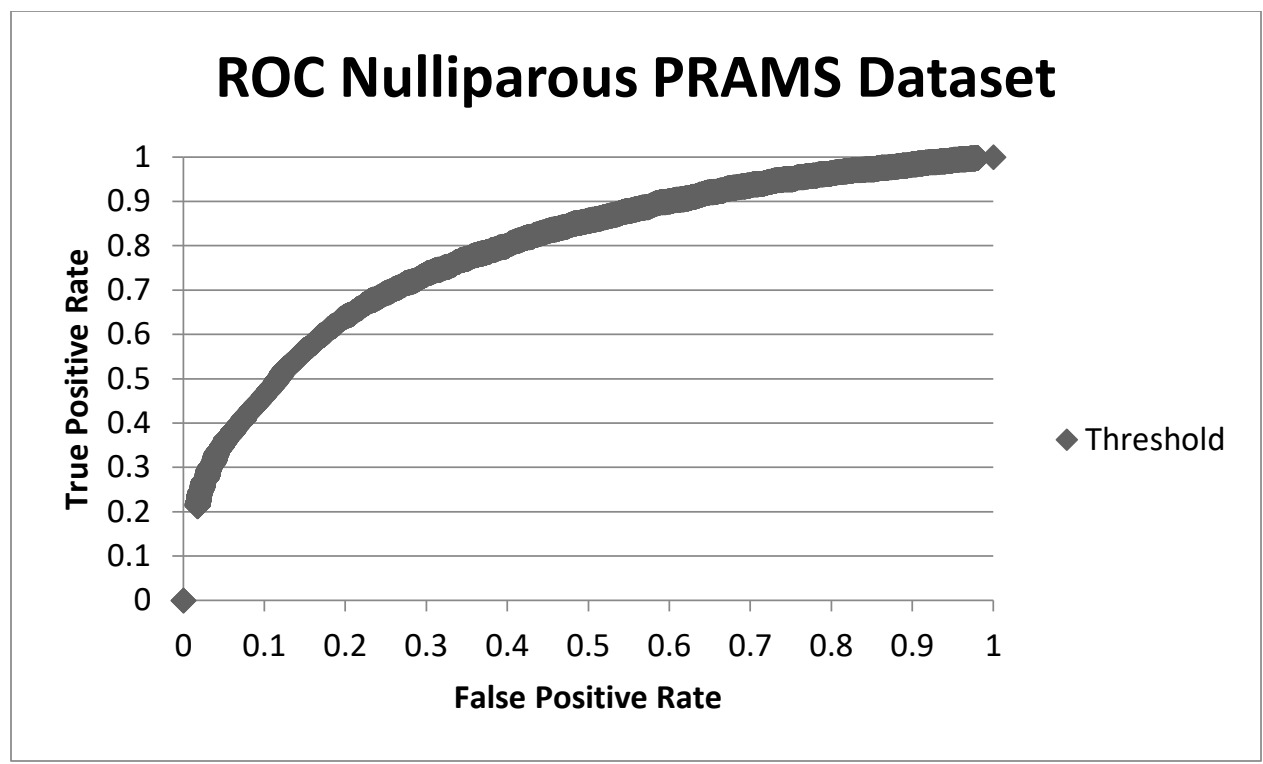

AUC: 0.788409

Figure 4.11. ROC Curve Performance for PRAMS Nulliparous Dataset

The parameters of the best final networks, which optimize sensitivity, for the BORN and PRAMS Dataset at 7.9\% Prevalence can be found in Appendix C. The final results of this research are summarized below in Tables 4.28-4.31.

\subsection{Comparison to Past Results}

Table 4.28. Display of the Artificial Neural Network results for BORN and PRAMS datasets

\begin{tabular}{|l|l|l|l|}
\hline \multicolumn{1}{|c|}{ Datasets } & \multicolumn{3}{c|}{ Current Research (2018) } \\
\cline { 2 - 4 } & Sensitivity & Specificity & AUC \\
\hline PRAMS_Parous & $84.1 \%$ & $71.4 \%$ & 0.8059 \\
\hline PRAMS_Nulliparous & $83.8 \%$ & $76.0 \%$ & 0.7884 \\
\hline BORN_Parous & $89.2 \%$ & $67.8 \%$ & 0.8944 \\
\hline BORN_Nulliparous & $89.0 \%$ & $71.5 \%$ & 0.8882 \\
\hline
\end{tabular}


Table 4.29. Displays the Artificial Neural Network results for past results (2015)

\begin{tabular}{|l|l|l|l|}
\hline \multicolumn{1}{|c|}{ Datasets } & \multicolumn{3}{c|}{ Past Research [24] } \\
\cline { 2 - 4 } & Sensitivity & Specificity & AUC \\
\hline PRAMS_Parous & $68.15 \%$ & $64.17 \%$ & 0.7256 \\
\hline PRAMS_Nulliparous & $40.35 \%$ & $94.75 \%$ & 0.7064 \\
\hline BORN_Parous & $50.53 \%$ & $91.61 \%$ & 0.7721 \\
\hline BORN_Nulliparous & $53.96 \%$ & $95.40 \%$ & 0.7970 \\
\hline
\end{tabular}

Table 4.30. Displays the Artificial Neural Network results for past results (2009)

\begin{tabular}{|l|l|l|l|}
\hline \multicolumn{1}{|c|}{ Datasets } & \multicolumn{3}{|c|}{ Past Research [27] } \\
\cline { 2 - 4 } & Sensitivity & Specificity & AUC \\
\hline PRAMS_Parous & $65.13 \%$ & $84.07 \%$ & 0.8195 \\
\hline PRAMS_Nulliparous & $61.08 \%$ & $71.14 \%$ & 0.7195 \\
\hline
\end{tabular}

Table 4.31. Displays the Artificial Neural Network results for past results (2007)

\begin{tabular}{|l|l|l|}
\hline \multicolumn{1}{|c|}{ Datasets } & \multicolumn{2}{|c|}{ Past Research [39] } \\
\cline { 2 - 3 } & Sensitivity & Specificity \\
\hline PRAMS & $65 \%$ & $84 \%$ \\
\hline
\end{tabular}




\subsection{Results and Discussion Summary}

This research focused on data preparation methods in comparison to past research which focused on the machine learning algorithms. The first step dealt with class imbalance (ubBalance [76]) reducing the dataset from 679,697 (BORN) and 109,079 (PRAMS) cases to 102,187 (BORN) and 46,867 (PRAMS) cases. This was a major change from past research [24], [27], [11], and resulted in faster computational time building the ANN models and improved accuracy of the classifier during training and testing. In addition, the second step of filling in missing values using a package in $\mathrm{R}$ (missForest [48]), proved faster than a case-based reasoning approach to fill in missing values. Ong remarked difficulty with comparing past results with her current methodology, due to a variety of changes: a new ANN tool, introducing 5-by-2 cross validation to reduce bias of results, prevalence to simulate real world conditions and the use of an updated version of the PRAMS database. Therefore, effectiveness of these two data preparation methods were reflected in the improved sensitivity results, when following the methodology of Ong [24] closely. In addition, this research used significantly fewer variables (17-22) than past research (34-45 for Ong [24] and 48 for Catley [39]). This addressed an area of improvement discussed by Ong, who suggested fewer variables might result in better results.

There were promising results when evaluating the BORN and PRAMS dataset. Out of all the datasets, the BORN_Parous dataset had the best results when considering classifier performance (AUC) and sensitivity values. The Parous datasets had higher sensitivity metrics in comparison to the Nulliparous datasets, these datasets contain features with prior medical history (i.e. previous premature birth) and this seems to have a positive affect on the classifier's ability to classify preterm birth. There was an overall increase in sensitivity when comparing to past 
results: $68.15 \%$ [24], 65\% [27], 65\% [39], meaning there are more accurate predictions when predicting preterm birth. Since, the objective of the thesis is to obtain the most accurate results when it comes to predicting preterm births, the specificity value is also important when referring to clinical costs and resources; however, it is not as critical as correctly predicting a premature birth outcome.

The aim was to exceed past sensitivity results and compare results to past performance metrics. The performance of the BORN and PRAMS database using the ANN Builder, did exceed past sensitivity results of prior students but did not surpass previous specificity metrics for all four datasets. One of the advantages of the ANN builder is its ability to optimize sensitivity or specificity; therefore, the model could be tailored specifically to fit the physician's needs, depending on which performance metric is desired. To be considered clinically useful in the context of this research a sensitivity of $65 \%$ and a specificity of $85 \%$ was recommended by our clinical partner [39], [24]. The sensitivity metric has been met and surpassed by all four datasets and future work will consist of improving the specificity metric to meet the clinical standards.

This chapter outlined the results obtained with this research work. The results of the methodology which focused on data preparation methods, showed an overall improvement in the sensitivity results of the Artificial Neural Network classifier. The reduced feature subsets for each of the four datasets were presented using the C5.0 DT Classifier. In addition, the performance metrics of the ANN classifier were displayed and compared to past work done in the MIRG lab. The contribution of this thesis work and future areas of improvement are discussed in the next chapter. 


\section{Chapter: Conclusion}

\subsection{Final Remarks and Conclusion}

This thesis work was focused on improving the sensitivity results of predicting a preterm birth outcome using existing machine learning tools, applied to two large population-based datasets. The method of improving the sensitivity results focused on preprocessing methods, primarily addressing missing values and class imbalances found in the clinical datasets. In addition, with the use of the C5.0 DT classifier, similar important features were identified between these widely different datasets. This is a positive result as it shows some congruence between various databases; these features will be necessary to include when implementing a similar database system in a clinical environment. By addressing these common data preprocessing concerns, this thesis work contributed to a higher accuracy and faster computational time when generating the ANN models.

\subsection{Contributions to Knowledge}

1. This work demonstrated that the predictive system described in this thesis could potentially be used in both an American (PRAMS) and a Canadian environment (BORN). This provides further evidence that this algorithm could be further developed to someday be incorporated into obstetrical clinics in Canada where prenatal data is collected prospectively.

2. New tools were used in this research work: R, (missForest and ubBalance) and Tableau which have not been used previously by students in the MIRG lab for data preprocessing methods. Using R for imputing missing values has greatly improved computational time, 
in comparison to previous methods (k-NN Algorithm). Tableau was an effective tool to visualize real-world data. In addition, this tool provided insight into the importance of some features over others, even before feature selection methods were applied. R and Tableau were chosen over other software tools such as SAS because these software tools needed to be open source to allow functionality within a clinical environment.

3. Similarly to previous students' work, the ANN classifier performed better with the BORN data than the PRAMS data [26], [29]. This may indicate that the variables collected by BORN may be more helpful to predict preterm births.

4. Previous work highlighted a need to attempt to reduce the number of variables used. This work has greatly reduced the number of features analyzed in the BORN (20 features for Parous and 17 features for Nulliparous) and PRAMS (22 features for Parous and 19 features for Nulliparous) in comparison to past work by Ong [24]: BORN (45 features for Parous and 38 features for Nulliparous) and PRAMS (48 features for Parous and 32 features for Nulliparous). This reduced feature subset maintained high sensitivity with reduced computational time.

5. These data preprocessing steps can be applied to variety of fields outside of the clinical sphere such as financial or environmental datasets with missing values; this is because these libraries in R are not specific to clinical data, are open-source and can handle large datasets.

6. Through data preprocessing methods described in the thesis, the sensitivity metric has surpassed previous methods, critical for predicting preterm births effectively; and the specificity metric has also remained sufficiently high. During the testing stage the 
prevalence was set to $7.9 \%$, to ensure that the data reflected an accurate population of preterm to term cases.

7. This work identified several similar features chosen by the DT classifier between both the PRAMS and BORN dataset:
a. Multiple gestation
b. Premature rupture of membranes
c. Small gestation age
d. Large gestational age
e. Mother's age in years
f. Number of previous preterm babies
g. Hypertension
h. Obstetrical complications (yes or no?)

These features might be important to maintaining high sensitivity results when moving the system to a real clinical environment.

8. During the feature selection methods, there were several features in the BORN dataset which were restricted from access due to data privacy concerns, such as geographical data; access to this data could result in an even higher sensitivity and specificity results for the classifier. As well as including shared features between BORN and PRAMS such as features including: hypertension, multiple gestation and maternal age. Given that the plan is to eventually integrate this classifier into a clinical environment in Canada, access to this data would be a great resource for future work. One option would be to anonymize the data so that researchers would have access to important information while still upholding the privacy of patients 
9. This developed system is non-invasive and has the capability to predict a preterm birth prior to 23 weeks, using only data; it surpasses the accuracy of the current gold standardfetal fibronectin.

\subsection{Future Work}

1. Future work should focus on improving the specificity performance metric (specifically dealing with the high number of false positives). There is an important trade-off between a high sensitivity and a high specificity. Although this work surpassed previous sensitivity results, the same was not true for the specificity metric. Specificity results were generally higher across the PRAMS datasets in comparison to BORN, therefore, certain features in PRAMS, were likely central to an increase in the specificity results. However, the specificity metric did not meet the clinical standard of $85 \%$. Including similar features to those reported in the PRAMS dataset might be a solution to increasing this specificity metric.

2. Future work will include testing and validating the results from the use of these data preprocessing software. Data will be removed and then added back into the dataset, to test the validity of using the missForest package in $\mathrm{R}$ for imputing missing values. Similarly, for ubUnder this can be tested by removing different percentages of the data (using this package left 50\% preterm and 50\% term cases in the dataset) to ensure this $50 \%$ split between preterm and term cases represents the highest accuracy for predicting preterm birth; further work can be tested by observing how $60 \%$ preterm and $40 \%$ term cases affects the overall accuracy.

3. Future works consist of building a dataset of clinical features collected at obstetrical clinics from past neonatal cases with known labels of preterm or term cases. To build this 
dataset, the similar features identified between the BORN and PRAMS dataset could be used to ensure high accuracy of the ANN classifier. Therefore, with future cases which contain unlabeled data, physicians will be able to make an accurate prediction on whether an individual may be at risk for a premature birth before 23 weeks gestation and then apply preventative care.

4. Investigate the effect of increasing the size of the testing set and see how the classifier performs. The classifier was tested in the final stage with 9210 term and 790 preterm cases from the BORN dataset. This testing set should be increased to test the stability of the performance of the classifier, with the same ratio between term and preterm cases. This can be done by randomly resampling the original 10,000 test set for an increased test set and then applying the classifier.

5. Eventually there will be a need to implement this system nationwide if it performs well in local clinics. Therefore, greater insight is needed into how this classifier will perform with data from populations in Canada, which have higher than normal preterm birth rates (rural and remote areas).

6. Future work could include integrating this predictive tool at a clinical site in conjunction with the prospective collection of data inputted into the Ontario Perinatal Record [37], through a secure web service. Preterm birth predictions made by the system could then be compared to the eventual pregnancy outcome, to determine the real-world accuracy of the prediction tool. 


\section{References}

[1] $\quad$ H. C. Koh and G. Tan, "Data mining applications in healthcare," Journal of healthcare information management, vol. 19, no 2, pp. 65-71, 2011.

[2] J. Engel, "GE, IBM Race to Deliver on A.I. Hype in Healthcare," Xconomy Boston, 26 June 2017. [Online]. Available: https://www.xconomy.com/boston/2017/06/26/ge-ibm-race-todeliver-on-a-i-hype-in-healthcare/. [Accessed 2018].

[3] S. Armstrong, "The computer will assess you now," BMJ, London, vol. 355, no. 1, pp. 5680, 2016.

[4] $\quad$ BORN, "BORN Information System," Better Outcomes and Registry \& Network, 2013. [Online]. Available: https://www.bornontario.ca/en/born-information-system/. [Accessed 2017].

[5] CDC, "Premature Birth," Center for Disease Control and Prevention, 2014. [Online]. Available: https://www.cdc.gov/prams/questionnaire.htm. [Accessed 2017].

[6] J. R. Quinlan, " C5. 0 Data Mining Tool. RuleQuest Research.," 1997. [Online]. Available: https://www.rulequest.com/see5-info.html [Accessed 2017].

[7] S. Nissen, "Implementation of a fast artificial neural network library (fann)," Department of Computer Science University of Copenhagen, Copenhagen, pp.1-92, 2003.

[8] J. Wang, "Data mining: opportunities and challenges,". IGI Global, pp. 85-105, 2003.

[9] N. Zhang and W. F. Lu, "An efficient data preprocessing method for mining customer survey data," Industrial Informatics, 2007 5th IEEE International Conference, vol. 1, pp. 573-578, 2007.

[10] S. García, J. Luengo and F. Herrera, "Data preprocessing in data mining," Switzerland. Springer International Publishing, pp. 195-243, 2015.

[11] C. Catley, M. Frize, R. C. Walker and D. C. Petriu, "Predicting high-risk preterm birth using artificial neural networks," IEEE transactions on information technology in biomedicine, vol. 10, no. 3, pp. 540-549, 2006.

[12] M. Frize, N. Yu and S. Weyand, "Effectiveness of a hybrid pattern classifier for medical applications," International Journal of Hybrid Intelligent Systems, vol. 8, no. 2, pp. 71-79, 2011.

[13] H. Martirosyan, M. Frize, D. E. Ong, J. Gilchrist and E. Bariciak, "A Decision-Support System for Expecting Mothers and Obstetricians," 6th European Conference of the International Federation for Medical and Biological Engineering. IFMBE Proceedings, vol. 45, pp. 703-706, 2015.

[14] $\quad$ K. M. Groom and P. R. Bennett, "Tocolysis for the treatment of preterm labour-a clinically based review.," The Obstetrician \& Gynaecologist, London, vol. 6, no. 1, pp. 4-11, 2004.

[15] S. Beck, D. Wojdyla, L. Say, A. P. Betran, M. Merialdi, J. H. Requejo and P. F. Van Look, "The worldwide incidence of preterm birth: a systematic review of maternal mortality and morbidity," Bulletin of the World Health Organization,vol. 88, pp. 31-38, 2010.

[16] BORN Ontario, "Perinatal Health Indicators for Ontario 2012," 2012. [Online]. Available: https://www.bornontario.ca/assets/documents/specialreports/Perinatal\%20Health\%20Indicators \%20for\%20Ontario\%202012.pdf.[Accessed 2018].

[17] R. L. Goldenberg, J. F. Culhane, J. D. Iams and R. Romero, "Epidemiology and causes of preterm birth," The lancet, vol. 371, no. 9606, pp. 75-84, 2008. 
[18] S. A. Leonard, C. M. Crespi, D. C. Gee, Y. Zhu and S. E. Whaley, "Prepregnancy risk factors for preterm birth and the role of maternal nativity in a low-income, hispanic population.," Maternal and child health journal, vol. 19, no. 10, pp. 2295-2302, 2015.

[19] P. J. Meis, R. L. Goldenberg, B. M. Mercer, J. D. Iams, A. H. Moawad, M. Miodovnik and A. Das, "The preterm prediction study: risk factors for indicated preterm births," American journal of obstetrics and gynecology, vol. 178, no. 3, pp. 562-567, 1998.

[20] M. Parisaei, J. Currie, N. O’Gorman, S. Morris and A. L. David, "Implementation of foetal fibronectin testing: Admissions, maternal interventions and costs at 1 year," Journal of Obstetrics and Gynaecology, vol. 36, no. 7, pp. 888-892, 2016.

[21] S. N. Deshpande, A. D. I. Van Asselt, F. Tomini, N. Armstrong, A. Allen, C. Noake and M. E. Westwood, "Rapid fetal fibronectin testing to predict preterm birth in women with symptoms of premature labour: a systematic review and cost analysis.," NIHR Journals Library, Southampton, vol. 40, no. 17, pp. 1-8, 2013.

[22] N. L. Hezelgrave and A. H. Shennan, "Quantitative fetal fibronectin to predict spontaneous preterm birth: a review.," Women's Health, vol. 12, no. 1, pp. 121-128, 2016.

[23] T. M. Malak, F. Sizmur, S. C. Bell and D. J. Taylor, "Fetal fibronectin in cervicovaginal secretions as a predictor of preterm birth," BJOG: An International Journal of Obstetrics \& Gynaecology, vol. 103, no. 7, pp. 648-653, 1996.

[24] D. E. Ong, "An Integrated Machine Learning Approach to Optimize the Estimation of Preterm Birth,". Carleton University, Ottawa, 2015.

[25] PHIP, " Personal Health Information Protection Act,"November 2014 [Online]. Available: https://www.ontario.ca/laws/statute/04p03?search=phipa.[Accessed 2017].

[26] T. M. Program, "BORN - Better Outcomes Registry and Network," 2013. [Online]. Available: https://www.themothersprogram.ca/resources-and-information/born-betteroutcomes-registry-and-network.[Accessed 2018].

[27] N. Yu, An integrated decision tree-artificial neural network hybrid to estimate clinical outcomes: ICU mortality and pre-term birth. Carleton University, Ottawa, 2009.

[28] G. S. Berkowitz, C. Blackmore-Prince, R. H. Lapinski and D. A. Savitz, "Risk factors for preterm birth subtypes.," Epidemiology, vol. 9, no. 3, pp. 279-285, 1998.

[29] P. J. Meis, R. L. Goldenberg, B. M. Mercer, J. D. Iams, A. H. Moawad, M. Miodovnik and A. Das, "The preterm prediction study: risk factors for indicated preterm births," American journal of obstetrics and gynecology, vol. 178, no. 3, pp. 562-567, 1998.

[30] L. J. Muglia and M. Katz, "The enigma of spontaneous preterm birth," New England Journal of Medicine, vol. 362, no. 6, pp. 529-535, 2010.

[31] G. Lim, J. Tracey, N. Boom, S. Karmakar, J. Wang, J. M. Berthelot and C. Heick, "CIHI survey: Hospital costs for preterm and small-for-gestational age babies in Canada," Healthcare Quarterly, vol. 12, no. 4, pp. 20-24, 2009.

[32] S. Saigal and L. W. Doyle, "An overview of mortality and sequelae of preterm birth from infancy to adulthood," The Lancet, vol. 371, no. 9608, pp. 261-269, 2008.

[33] T. Yoshizato, H. Obama, T. Nojiri, Y. Miyake, S. Miyamoto and T. Kawarabayashi, "Clinical significance of cervical length shortening before 31 weeks' gestation assessed by longitudinal observation using transvaginal ultrasonography," Journal of Obstetrics and Gynaecology Research, vol. 34, no. 5, pp. 805-811, 2008.

[34] W. L. Maner, R. E. Garfield, H. Maul, G. Olson and G. Saade, "Predicting term and preterm delivery with transabdominal uterine electromyography.," Obstetrics \& Gynecology,vol. 101, no.6, pp. 1254-1260, 2003.

[35] H. Leitich and A. Kaider, "Fetal fibronectin - how useful is it in the prediction of preterm birth?," BJOG: An International Journal of Obstetrics \& Gynaecology, vol. 110, no .20, pp. 66$70,2003$. 
[36] M. Frize, E. Bariciak, S. Dunn, S. Weyand, J. Gilchrist and S. Tozer, "Combined PhysicianParent Decision Support tool for the neonatal intensive care unit.," medical measurements and applications proceedings (MeMeA), vol. 197, pp. 59-64, 2011.

[37] Provincial Council for Maternal and Child Health, "A User Guide to the Ontario Perinatal Record.," 2017. [Online]. Available: http://www.pcmch.on.ca/wpcontent/uploads/2017/11/OPR_UserGuide_2017OCT26.pdf. [Accessed 2017].

[38] P. H. Shiono and M. A. Klebanoff, "A review of risk scoring for preterm birth," Clinics in perinatology, vol. 20, no. 1, pp. 107-125, 1993.

[39] C. Catley, An Integrated Hybrid Data Mining System for Preterm Birth Risk Assessment Based on a Semantic Web Servcies for Healthcare Framework, Carleton University, Ottawa, 2007.

[40] J. R. Quinlan, "Bagging, boosting, and C4. 5.," AAAI/IAAI, vol. 1, pp. 725-730, 1996.

[41] W. L. Maner and R. E. Garfield, "Identification of human term and preterm labor using artificial neural networks on uterine electromyography data," Annals of biomedical engineering, vol. 35, no. 3, pp. 465-473, 2007.

[42] $\quad$ C. J. Lockwood and E. Kuczynski, "Risk stratification and pathological mechanisms in preterm delivery," Paediatric and perinatal epidemiology, vol. 15, pp. 78-89, 2001.

[43] H. Y. Chen, C. H. Chuang, Y. J. Yang and T. P. Wu, "Exploring the risk factors of preterm birth using data mining.," Expert systems with applications, vol. 38, no. 5, pp. 5384-5387, 2011.

[44] H. Kang, "The prevention and handling of the missing data," Korean journal of anesthesiology, vol. 64, no. 5, pp. 402-406, 2013.

[45] G. Doquire and M. Verleysen, "Feature selection with missing data using mutual information estimators," Neurocomputing, vol. 90, pp. 3-11, 2012.

[46] A. B. Pedersen, E. M. Mikkelsen, D. Cronin-Fenton, N. R. Kristensen, T. M. Pham, L. Pedersen and I. Petersen, "Missing data and multiple imputation in clinical epidemiological research," Clinical epidemiology, vol. 9, pp. 157-166, 2017.

[47] A. K. Waljee, A. Mukherjee, A. G. Singal, Y. Zhang, J. Warren, U. Balis and P. D. Higgins, "Comparison of imputation methods for missing laboratory data in medicine," BMJ open, vol. 3, no. 8, pp.1-7, 2013.

[48] D. J. Stekhoven and P. Bühlmann, "MissForest—non-parametric missing value imputation for mixed-type data.," Bioinformatics, vol. 28, no. 1, pp. 112-118, 2011.

[49] S. V. Buuren and K. Groothuis-Oudshoorn, "mice: Multivariate imputation by chained equations in R," Journal of statistical software, vol. 10, no. 2, pp. 1-68, 2010.

[50] M. J. Azur, E. A. Stuart, C. Frangakis and P. J. Leaf, "Multiple imputation by chained equations: what is it and how does it work?," International journal of methods in psychiatric research, vol. 20, no. 1, pp. 40-49, 2011.

[51] J. A. Sterne, I. R. White, J. B. Carlin, M. Spratt, P. Royston, M. G. Kenward and J. R. Carpenter, "Multiple imputation for missing data in epidemiological and clinical research: potential and pitfalls," vol. 339, no. 7713, pp. 157-160, Bmj, 2009.

[52] D. C. Li, C. W. Liu and S. C. Hu, "A learning method for the class imbalance problem with medical data sets.," Computers in biology and medicine, vol. 40, no. 5, pp. 509-518, 2010.

[53] R. Longadge and S. Dongre, "Class imbalance problem in data mining review," vol. 2, no .1, pp. 1-6, 2013.

[54] M. A. Mazurowski, P. A. Habas, J. M. Zurada, J. Y. Lo, J. A. Baker and G. D. Tourassi, "Training neural network classifiers for medical decision making: The effects of imbalanced datasets on classification performance," Neural networks,vol. 21, no. 2-3, pp. 427-436, 2008.

[55] R. Longadge and S. Dongre, "Class imbalance problem in data mining review," International Journal of Computer Science and Network, vol. 2, no.1, pp. 1-6, 2013. 
[56] $\quad$ M. M. Rahman and D. N. Davis, "Addressing the class imbalance problem in medical datasets.," International Journal of Machine Learning and Computing, vol. 3, no. 2, pp. 224, 2013.

[57] V. Ganganwar, "An overview of classification algorithms for imbalanced datasets.," International Journal of Emerging Technology and Advanced Engineering, vol. 2. no. 4, pp. 4247, 2012.

[58] M. Vihinen, "How to evaluate performance of prediction methods? Measures and their interpretation in variation effect analysis," BMC genomics, vol 13. no. 4, pp. 1-10, 2012.

[59] A. H. Fielding and J. F. Bell, "A review of methods for the assessment of prediction errors in conservation presence/absence models," Environmental conservation, vol. 24, no. 1, pp. 38-49, 1997.

[60] J. Gilchrist, C. M. Ennett, M. Frize and E. Bariciak, "Neonatal mortality prediction using realtime medical measurements," medical measurements and applications proceedings (MeMeA), pp. 65-70, 2011.

[61] B. Vidakovic, "Statistics for bioengineering sciences: with MATLAB and WinBUGS support," Springer Science \& Business Media., pp. 471-472. 2011.

[62] J. N. Mandrekar, "Receiver operating characteristic curve in diagnostic test assessment," Journal of Thoracic Oncology, vol. 5, no. 9, pp. 1315-1316, 2010.

[63] S. Boughorbel, F. Jarray and M. El-Anbari, "Optimal classifier for imbalanced data using Matthews Correlation Coefficient metric," PloS one, vol 12. no. 6, pp.1-17, 2017.

[64] MathWorks, "Normalize," R2015b. 2015 [Online]. Available: https://www.mathworks.com/help/matlab/ref/normalize.html\#d119e785470.[Accessed 2017]

[65] S. R. Maetschke, P. B. Madhamshettiwar, M. J. Davis and M. A. Ragan, "Supervised, semisupervised and unsupervised inference of gene regulatory networks," Briefings in bioinformatics, vol. 15, no. 2, pp. 195-211, 2013.

[66] S. B. Kotsiantis, I. Zaharakis and P. Pintelas, "Supervised machine learning: A review of classification techniques," Emerging artificial intelligence applications in computer engineering, vol. 160, pp. 3-24, 2007.

[67] A. Abraham, F. Pedregosa, M. Eickenberg, P. Gervais, A. Mueller, J. Kossaifi and G. Varoquaux, "Machine learning for neuroimaging with scikit-learn," Frontiers in neuroinformatics, vol. 8, pp. 1-10, 2014.

[68] Z. Ghahramani, "Unsupervised learning," Advanced lectures on machine learning , pp. 72-112, 2004.

[69] J. H. Krijthe, "RSSL: Semi-supervised Learning in R," International Workshop on Reproducible Research in Pattern Recognition, pp. 104-115, 2016.

[70] S. García, J. Luengo and F. Herrera, Data preprocessing in data mining, Granada: Springer International Publishing, pp. 195-243, 2015.

[71] G. P. Zhang, "Neural networks for classification: a survey," IEEE Transactions on Systems, Man, and Cybernetics, Part C (Applications and Reviews), vol. 30, no. 4, pp. 451-462, 2000.

[72] A. S. Galathiya, A. P. Ganatra and C. K. Bhensdadia, "Improved decision tree induction algorithm with feature selection, cross validation, model complexity and reduced error pruning.," International Journal of Computer Science and Information Technologies, vol. 3, no. 2, pp. 3427-3431, 2012.

[73] I. G. Maglogiannis, Emerging artificial intelligence applications in computer engineering: real word ai systems with applications in ehealth, hci, information retrieval and pervasive technologies, Amsterdam: IOS Press, vol. 160, pp. 3-25, 2007.

[74] R. Pandya and J. Pandya, "C5. 0 algorithm to improved decision tree with feature selection and reduced error pruning.," International Journal of Computer Applications, vol. 117, no. 16, pp. $18-21,2015$. 
\begin{tabular}{l|l} 
[75] & P. H. Swain and H. Hauska, "The decision tree classifier: Design and potential," IEEE
\end{tabular} Transactions on Geoscience Electronics, vol. 15, no. 3, pp. 142-147, 1977.

[76] D. Pozzolo, O. Caelen and B. Gianluca, "Package 'unbalanced," 26 June 2015. [Online]. Available: https://cran.r-project.org/web/packages/unbalanced/unbalanced.pdf.[Accessed 2018].

[77] M. Misztal, "Some Remarks on the Data Imputation Using “missForest” Method.,"pp. 1-11, 2013.

[78] A. T. Azar, H. I. Elshazly, A. E. Hassanien and A. M. Elkorany, "A random forest classifier for lymph diseases.," Computer methods and programs in biomedicine, vol. 2, no. 113, pp. 465 473, 2014.

[79] C. Nguyen, Y. Wang and H. N. Nguyen, "). Random forest classifier combined with feature selection for breast cancer diagnosis and prognostic," Journal of Biomedical Science and Engineering, vol. 5, no. 6, p. 551, 2013.

[80] B. L. Kalman and S. C. Kwasny, "Why tanh: choosing a sigmoidal function," Neural Networks, vol. 4, pp. 578-581, 1992.

[81] J. V. Tu, "Advantages and disadvantages of using artificial neural networks versus logistic regression for predicting medical outcomes," Journal of clinical epidemiology, vol. 11, no. 49, pp. 1225-1231, 1996.

[82] Tableau, "Tableau Server 10.3.6," 12 December 2017. [Online]. Available: https://www.tableau.com/support/releases/server/10.3.6.[Accessed 2018].

[83] Cygwin. [Online]. Available: https://www.cygwin.com/.[Accessed 2017].

[84] J. R. Quinlan, "C5.0: An Informal Tutorial," RULEQUEST RESEARCH 2017, [Online]. Available: https://www.rulequest.com/see5-unix.html.[Accessed 2018].

[85] C. M. Ennett, M. Frize and E. Charette, "Improvement and automation of artificial neural networks to estimate medical outcomes.," Medical engineering \& physics, vol. 26, no. 4, pp. 321-328, 2004.

[86] D. Rybchynski, "Design of an artificial neural network research framework to enhance the development of clinical prediction models," University of Ottawa, Ottawa, 2005.

[87] A. S. Miller and B. H. Blott, "Review of neural network applications in medical imaging and signal processing.," Medical and Biological Engineering and Computing, vol. 30, no. 5, pp. 449-464, 1992.

[88] J. Jiang, P. Trundle and J. Ren, "Medical image analysis with artificial neural networks," Computerized Medical Imaging and Graphics, vol. 34, no. 8, pp. 617-631, 2010.

[89] S. A. Durai and E. A. Saro, "Image compression with back-propagation neural network using cumulative distribution function," World Academy of Science, Engineering and Technology, vol. 17, pp. 60-64, 2006.

[90] M. Frize, J. Gilchrist, H. Martirosyan and E. Bariciak, "Integration of outcome estimations with a clinical decision support system: Application in the neonatal intensive care unit (NICU)," MeMeA, pp. 175-179, 2015.

[91] J. Honaker, G. King, M. Blackwell and M. M. Blackwell, "Package "Amelia'.," 2010. [Online]. Available: http://kambing.ui.ac.id/cran/web/packages/Amelia/Amelia.pdf.[Accessed 2018]. 


\section{Appendices}

Appendix A-Ethics Approval Form

Appendix B- Description of BORN and PRAMS Features

Appendix C- Description of ANN Final Network Parameters 


\section{Appendix A-Ethics Approval Form}

\section{External Researcher Data Sharing Agreement}

\section{CDC PRAMS AGREEMENT FOR SHARING MULTI-STATE DATA WITH EXTERNAL RESEARCHERS}

Pregnancy Risk Assessment Monitoring as principal investigator(coinvestigator on this proposed analysis of PRAMS data and assure compliance with the requiram) data, agree to the following requirements for the use of agreement. 2. I will not use nor permit approved collaborators and staff to use these data to conduct analyses other than
those described in the proposal.

3. I will not release the data set or any part of it to any person other than those listed as collaborators in the
attached proposal. I will assure that all approved collaborators understand that they ma not shase attached proposal. I will assure that all approved collaborators understand that they may not share the
data set or any part of it.

4. I will neither attempt, nor permit others to attempt, to use the data set or link it with other data sets to will not use and/or distribute this information, nor will I permit others to the CDC PRAMS staff at PRAMSProposals@cdc. identifiable data in the e-mail, so they can prevent future disco discovery but will not disclose any members of my team will inform anyone else of this knowledge.

5. All aral or written presentations of the results of the analyses will include an acknowledgment of the PRAMS Working Group and the Centers for Disease Control and Prevention (CDC). 6. All oral or written presentations of the results of the analyses will be submitted to the CDC at least 3
weeks prior to presentation or submission to a journal so presentations can be forwarded to the PRAM presentation/manuscript to the author. The states will have two weeks to submit comments on the publication. 7. CDC PRAMS staff and staff from states whose data were used in the analysis will be notified upon final
publication of an article and provided with citation information. 8. When the proposed analyses are completed, all copies of these data will be destroyed (confirmed in
writing to PRAMSProposals@cdc.gov) or returned to CDC.

My signature and the signatures of all co-investigators indicate our agreement to comply with these requirements.

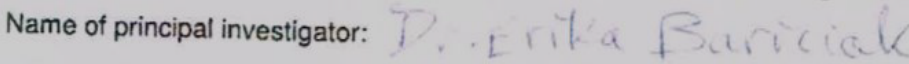

Title and Organization:

Signature:

Name of collaborator:
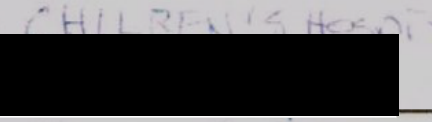

Signature:

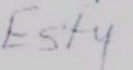

Date:
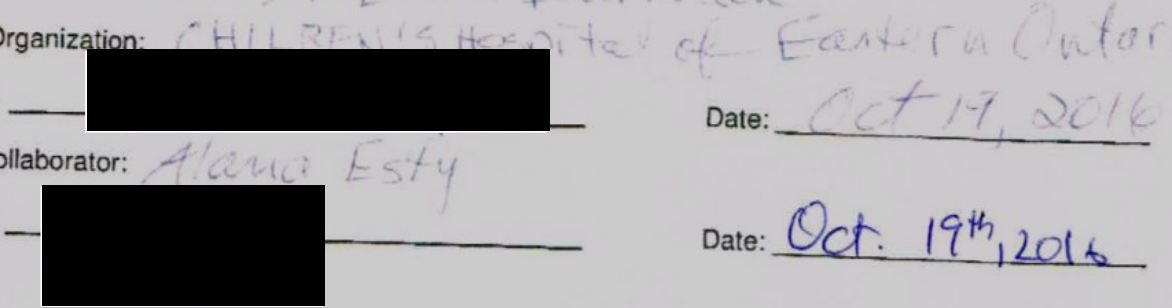


\section{Appendix B - BORN and PRAMS Features}

BORN Parous Features

\begin{tabular}{|c|c|}
\hline 1. MULTGEST & 2. MATAGE \\
\hline 3. PPRETERM & 4. PTERM \\
\hline 5. PARITY & 6. PRESENT \\
\hline 7. DELTYPE & 8. PREVCS \\
\hline 9. ANTESTER & 10. GENDER \\
\hline 11. APGAR1 & 12. APGAR5 \\
\hline 13. INTBF & 14. SMOKING \\
\hline 15. MATHP0 & 16. MATHP1 \\
\hline 17. MATHP2 & 18. MATHP3 \\
\hline 19. MATHP4 & 20. MATHP5 \\
\hline 21. MATHP16 & 22. MATHP17 \\
\hline 23. MATHP18 & 24. MATHP19 \\
\hline 25. MATHP26 & 26. MATHP27 \\
\hline 27. OBCOMP0 & 28. OBCOMP1 \\
\hline 29. OBCOMP2 & 30. OBCOMP3 \\
\hline 31. OBCOMP4 & 32. OBCOMP5 \\
\hline 33. OBCOMP6 & 34. OBCOMP7 \\
\hline 35. OBCOMP8 & 36. OBCOMP9 \\
\hline 37. OBCOMP10 & 38. OBCOMP11 \\
\hline 39. OBCOMP12 & 40. OBCOMP13 \\
\hline
\end{tabular}




\begin{tabular}{|c|c|}
\hline 41. OBCOMP14 & 42. OBCOMP15 \\
\hline 43. REPASS & 44. FIRSTVIS \\
\hline 45. CONGAN0 & 46. CONGAN52 \\
\hline 47. CONGAN54 & 48. CONGAN55 \\
\hline 49. DISCHTO & 50. PRENCLAS \\
\hline 51. GBSRES & 52. FISCALYEAR \\
\hline 53. LANGUAGE_up & 54. MATHP_sub \\
\hline 55. MATHP_ment & 56. CONGAN_CNS \\
\hline 57. CONGAN_EYE & 58. CONGAN_OROFACIAL \\
\hline 59. CONGAN_CARDIAC & 60. CONGAN_RES \\
\hline 61. CONGAN_GAS & 62. CONGAN_GEN \\
\hline 63. CONGAN_MUS & 64. CONGAN_CHR \\
\hline 65. Total_15_marital & 66. Single \\
\hline 67. not_separated & 68. separated \\
\hline 69. divorced & 70. widowed \\
\hline 71. Total_15_common_law & 72. Not_common_law \\
\hline 73. In_common_law & 74. Total_families_1 \\
\hline 75. Size_2_person & 76. Size_3_person \\
\hline 77. Size_4_person & 78. Size_5_or_more \\
\hline 79. Total_families_2 & 80. Total_couple_families \\
\hline 81. Married_couples & 82. Without_children_at_home \\
\hline 83. With_children_at_home & 84. child_1 \\
\hline 85. children_2 & 86. children_3_over \\
\hline
\end{tabular}




\begin{tabular}{|c|c|}
\hline 87. Common_law_couples & 88. Without_children_at_home 1 \\
\hline 89. With_children_at_home1 & 90. child1_1 \\
\hline 91. children1_2 & 92. children1_3_over \\
\hline 93. Total_lone_parent_families & 94. Female parent \\
\hline 95. child2_1 & 96. children2_2 \\
\hline 97. children2_3_over & 98. Male_parent \\
\hline 99. child3_1 & children3_2 \\
\hline children3_3_over & Average_number_children \\
\hline Total_family_by_type & One_family \\
\hline Multiple_family & Non_family \\
\hline Total_by_mother_tongue & Single_responses \\
\hline English & French \\
\hline Non_official_languages & Total_by_immigrant \\
\hline Non_immigrants & Immigrants \\
\hline Total by Aboriginal & Total_Aboriginal \\
\hline North_American_single & Metis_single_response \\
\hline Inuit_single_response & Multiple_Aboriginal \\
\hline Aboriginal_responses & Non_Aboriginal \\
\hline Total_by_labour & In_labour_force \\
\hline Employed & Unemployed \\
\hline Not_in_labour_force & Total_by_class \\
\hline Class_worker_NA & All_classes_worker \\
\hline Paid workers & Employees \\
\hline
\end{tabular}




\begin{tabular}{|c|c|c|c|}
\hline 133. & Self_employed & 134. & Without_paid_help \\
\hline 135. & With_paid_help & 136. & Self_employed_unco \\
\hline 137. & Without paid help2 & 138. & With paid help2 \\
\hline 139. & Unpaid_family_workers & 140. & Total_15_24_diploma \\
\hline 141. & No_diploma & 142. & Certificate_diploma \\
\hline 143. & High_school & 144. & Apprenticeship \\
\hline 145. & College_CEGEP & 146. & University_diploma \\
\hline 147. & diploma_or_below & 148. & degree \\
\hline 149. & Bachelor_degree & 150. & above bachelor \\
\hline 151. & Degree_in_medicine & 152. & Master_degree \\
\hline 153. & Doctorate & 154. & Total_25_to_64_diploma \\
\hline 155. & No_diploma_degree & 156. & Certi_diploma_degree \\
\hline 157. & High_school_certi & 158. & Apprenticeship_diploma \\
\hline 159. & College_CEGEP_or_other & 160. & University_dipoma \\
\hline 161. & diploma_below_bachelor & 162. & University_certificate \\
\hline 163. & Bachelor_s_degree & 164. & above_bachelor_degree \\
\hline 165. & Degree_medicine & 166. & Master_s_degree \\
\hline 167. & Earned_doctorate & 168. & Total_minority \\
\hline 169. & Total_visible_minority & 170. & Total_by_ethnic \\
\hline 171. & British_Isles_origins & 172. & French_origins \\
\hline 173. & Aboriginal_origins & 174. & Other_American_origins \\
\hline 175. & Caribbean_origins & 176. & Latin_Central \\
\hline 177. & European origins & 178. & African origins \\
\hline
\end{tabular}




\begin{tabular}{|cl|cl|}
\hline 179. & Arab_origins & 180. & West_Asian_origins \\
\hline 181. & South_Asian_origins & 182. & East_Asian_origins \\
\hline 183. & Oceania_origins & 184. & Family_income_2005 \\
\hline 185. & Under_10_000 & 186. & IN_10_000_to_19_999 \\
\hline 187. & IN_20_000_to_29_999 & 188. & IN_30_000_to_39_999 \\
\hline 189. & IN_40_000_to_49_999 & 190. & IN_50_000_to_59_999 \\
\hline 191. & IN_60_000_to_69_999 & 192. & IN_70_000_to_79_999 \\
\hline 193. & IN_80_000_to_89_999 & 194. & IN_90_000_to_99_999 \\
\hline 195. & IN_100_000_and_over & 196. & Median_family_income \\
\hline 197. & Average_family_income & 198. & Std_of_average_family \\
\hline 199. & Average_value_of_dwelling & 200. & OUTCOME \\
\hline
\end{tabular}

\section{$\underline{\text { BORN Nulliparous Features }}$}

\begin{tabular}{|l|l|}
\hline 1. MULTGEST & 2. MATAGE \\
\hline 3. PARITY & 4. PRESENT \\
\hline 5. DELTYPE & 6. PREVCS \\
\hline 7. ANTESTER & 8. GENDER \\
\hline 9. APGAR1 & 10. APGAR5 \\
\hline 11. INTBF & 12. SMOKING \\
\hline 13. MATHP0 & 14. MATHP1 \\
\hline 15. MATHP2 & 16. MATHP3 \\
\hline 17. MATHP4 & 18. MATHP5 \\
\hline
\end{tabular}




\begin{tabular}{|c|c|}
\hline 19. MATHP16 & 20. MATHP17 \\
\hline 21. MATHP18 & 22. MATHP19 \\
\hline 23. MATHP26 & 24. MATHP27 \\
\hline 25. OBCOMP0 & 26. OBCOMP1 \\
\hline 27. OBCOMP2 & 28. OBCOMP3 \\
\hline 29. OBCOMP4 & 30. OBCOMP5 \\
\hline 31. OBCOMP6 & 32. OBCOMP7 \\
\hline 33. OBCOMP8 & 34. OBCOMP9 \\
\hline 35. OBCOMP10 & 36. OBCOMP11 \\
\hline 37. ОВСОМР 12 & 38. OBCOMP13 \\
\hline 39. OBCOMP14 & 40. OBCOMP15 \\
\hline 41. REPASS & 42. FIRSTVIS \\
\hline 43. CONGAN0 & 44. CONGAN52 \\
\hline 45. CONGAN54 & 46. CONGAN55 \\
\hline 47. DISCHTO & 48. PRENCLAS \\
\hline 49. GBSRES & 50. FISCALYEAR \\
\hline 51. LANGUAGE_up & 52. MATHP_sub \\
\hline 53. MATHP_ment & 54.CONGAN_CNS \\
\hline 55. CONGAN_EYE & 56. CONGAN_OROFACIAL \\
\hline 57. CONGAN_CARDIAC & 58. CONGAN_RES \\
\hline 59. CONGAN_GAS & 60. CONGAN_GEN \\
\hline 61. CONGAN_MUS & 62. CONGAN_CHR \\
\hline 63. Total_15_marital & 64. Single \\
\hline
\end{tabular}




\begin{tabular}{|c|c|}
\hline 65. not_separated & 66. separated \\
\hline 67. divorced & 68. widowed \\
\hline 69. Total 15 common law & 70. Not common law \\
\hline 71. In_common_law & 72. Total_families_1 \\
\hline 73. Size_2_person & 74. Size_3_person \\
\hline 75. Size_4_person & 76. Size_5_or_more \\
\hline 77. Total_families_2 & 78. Total_couple_families \\
\hline 79. Married_couples & 80. Without_children_at_home \\
\hline 81. Common_law_couples & 82. Without_children_at_home1 \\
\hline 83. Total_lone_parent_families & 84. Female_parent \\
\hline 85. Male_parent & \\
\hline 86. Total_family_by_type & 87. One_family \\
\hline 88. Multiple_family & 89. Non_family \\
\hline 90. Total_by_mother_tongue & 91. Single_responses \\
\hline 92. English & 93. French \\
\hline 94. Non_official_languages & 95. Total_by_immigrant \\
\hline 96. Non_immigrants & 97. Immigrants \\
\hline 98. Total_by_Aboriginal & 99. Total_Aboriginal \\
\hline North_American_single & Metis_single_response \\
\hline Inuit_single_response & 103. Multiple_Aboriginal \\
\hline Aboriginal_responses & Non_Aboriginal \\
\hline Total_by_labour & 107. In_labour_force \\
\hline Employed & Unemployed \\
\hline
\end{tabular}




\begin{tabular}{|c|c|c|c|}
\hline 110. & Not_in_labour_force & 111. & Total_by_class \\
\hline 112. & Class_worker_NA & 113. & All_classes_worker \\
\hline 114. & Paid workers & 115. & Employees \\
\hline 116. & Self_employed & 117. & Without_paid_help \\
\hline 118. & With_paid_help & 119. & Self_employed_unco \\
\hline 120. & Without_paid_help2 & 121. & With_paid_help2 \\
\hline 122. & Unpaid_family_workers & 123. & Total_15_24_diploma \\
\hline 124. & No_diploma & 125. & Certificate_diploma \\
\hline 126. & High_school & 127. & Apprenticeship \\
\hline 128. & College_CEGEP_ & 129. & University_diploma \\
\hline 130. & diploma_or_below & 131. & degree \\
\hline 132. & Bachelor_degree & 133. & above_bachelor \\
\hline 134. & Degree_in_medicine & 135. & Master_degree \\
\hline 136. & Doctorate & 137. & Total_25_to_64_diploma \\
\hline 138. & No_diploma_degree & 139. & Certi_diploma_degree \\
\hline 140. & High_school_certi & 141. & Apprenticeship_diploma \\
\hline 142. & College_CEGEP_or_other & 143. & University_dipoma \\
\hline 144. & diploma_below_bachelor & 145. & University_certificate \\
\hline 146. & Bachelor_s_degree & 147. & above_bachelor_degree \\
\hline 148. & Degree_medicine & 149. & Master_s_degree \\
\hline 150. & Earned_doctorate & 151. & Total_minority \\
\hline 152. & Total_visible_minority & 153. & Total_by_ethnic \\
\hline 154. & British Isles origins & 155. & French origins \\
\hline
\end{tabular}




\begin{tabular}{|cl|cl|}
\hline 156. & Aboriginal_origins & 157. & Other_American_origins \\
\hline 158. & Caribbean_origins & 159. & Latin_Central \\
\hline 160. & European_origins & 161. & African_origins \\
\hline 162. & Arab_origins & 163. & West_Asian_origins \\
\hline 164. & South_Asian_origins & 165. & East_Asian_origins \\
\hline 166. & Oceania_origins & 167. & Family_income_2005 \\
\hline 168. & Under_10_000 & 169. & IN_10_000_to_19_999 \\
\hline 170. & IN_20_000_to_29_999 & 171. & IN_30_000_to_39_999 \\
\hline 172. & IN_40_000_to_49_999 & 173. & IN_50_000_to_59_999 \\
\hline 174. & IN_60_000_to_69_999 & 175. & IN_70_000_to_79_999 \\
\hline 176. & IN_80_000_to_89_999 & 177. & IN_90_000_to_99_999 \\
\hline 178. & IN_100_000_and_over & 179. & Median_family_income \\
\hline 180. & Average_family_income & 181. & Std_of_average_family \\
\hline 182. & Average_value_of_dwelling & 183. & OUTCOME \\
\hline
\end{tabular}

\section{$\underline{\text { PRAMS Parous Features }}$}

\begin{tabular}{|l|l|}
\hline 1. ID & 2. B_ORDER \\
\hline 3. CIG_1TRI & 4. CIG_2TRI \\
\hline 5. CIG_3TRI & 6. CIG_PRIOR \\
\hline 7. DEFECT & 8. FRACE_AMI \\
\hline 9. FRACE_ASN_NAPHSIS & 10. FRACE_BLK \\
\hline
\end{tabular}




\begin{tabular}{|c|c|}
\hline 11. FRACE_CHN & 12. FRACE_FLP \\
\hline 13. FRACE_JPN & 14. FRACE_NHW \\
\hline 15. FRACE_WHT & 16. HISP_BC \\
\hline 17. INFER_TR & 18. KESSNER \\
\hline 19. LGA & 20. MACROSOMIA \\
\hline 21. MARRIED & 22. MAT_AGE_NAPHSIS \\
\hline 23. MAT_ED & 24. MAT_RACE \\
\hline 25. MAT_TRAN & 26. MAT_WIC \\
\hline 27. MM_DIAB & 28. MM_FEVER \\
\hline 29. MM_HBP & 30. MM_LMP \\
\hline 31. MM_NOMD & 32. MM_PCV \\
\hline 33. MM_PROM & 34. MOMCIG \\
\hline 35. MOMLBS & 36. MOMSMOKE \\
\hline 37. MRACE_AMI & 38. MRACE_ASN_NAPHSIS \\
\hline 39. MRACE_BLK & 40. MRACE_CHN \\
\hline 41. MRACE_FLP & 42. MRACE_JPN \\
\hline 43. MRACE_NHW & 44. MRACE_OTH \\
\hline 45. MRACE_WHT & 46. OTH_TERM \\
\hline 47. PAT_ED & 48. PAY \\
\hline 49. PLURAL & 50. PNC_MTH \\
\hline 51. PNC_VST_NAPHSI.S & 52. PRE_LB_NAPHSIS \\
\hline 53. P_PRTERM & 54. SEX \\
\hline 55. SGA_10 & 56.SGA_2SD \\
\hline
\end{tabular}




\begin{tabular}{|l|l|}
\hline 57. YY4_LMP & 58. YY4_PCV \\
\hline 59. YY_LMP & 60. HISPANIC \\
\hline 61. URB_RUR & 62. BC_YRLLB \\
\hline 63. DRK63B_A & 64. DRK63L_A \\
\hline 65. DRK6C_PG & 66. INCOME5 \\
\hline 67. MOM_BMI & 68. MOM_BMIG \\
\hline 69. MOM_HT_I & 70. MOM_WT \\
\hline 71. PNCNO & 72. PNC_1TRM \\
\hline 73. PNC_WKS & 74. PP_NUMB \\
\hline 75. PREGHX & 76. SMK6C_PG \\
\hline 77. SMK6C_PP & 78. STRS_TT3 \\
\hline 79. STRS_T_G & 80. SURE_WKS \\
\hline 81. OUTCOME & \\
\cline { 1 - 2 } & \\
\hline
\end{tabular}

PRAMS Nulliparous Features

\begin{tabular}{|l|l|}
\hline 1. ID & 2. CIG_1TRI \\
\hline 3. CIG_2TRI & 4. CIG_3TRI \\
\hline 5. CIG_PRIOR & 6. DEFECT \\
\hline 7. FRACE_AMI & 8. FRACE_ASN_NAPHSIS \\
\hline 9. FRACE_BLK & 10. FRACE_CHN \\
\hline 11. FRACE_FLP & 12. FRACE_JPN \\
\hline
\end{tabular}




\begin{tabular}{|c|c|}
\hline 13. FRACE_NHW & 14. FRACE_WHT \\
\hline 15. HISP_BC & 16. INFER_TR \\
\hline 17. KESSNER & 18. LGA \\
\hline 19. MACROSOMIA & 20. MARRIED \\
\hline 21. MAT_AGE_NAPHSIS & 22. MAT_ED \\
\hline 23. MAT_RACE & 24. MAT_TRAN \\
\hline 25. MAT_WIC & 26. MM_DIAB \\
\hline 27. MM_FEVER & 28. MM_HBP \\
\hline 29. MM_LMP & 30. MM_NOMD \\
\hline 31. MM_PCV & 32. MM_PROM \\
\hline 33. MOMCIG & 34. MOMLBS \\
\hline 35. MOMSMOKE & 36. MRACE_AMI \\
\hline 37. MRACE_ASN_NAPHSIS & 38. MRACE_BLK \\
\hline 39. MRACE_CHN & 40. MRACE_FLP \\
\hline 41. MRACE_JPN & 42. MRACE_NHW \\
\hline 43. MRACE_OTH & 44. MRACE_WHT \\
\hline 45. PAT_ED & 46. PAY \\
\hline 47. PLURAL & 48. PNC_MTH \\
\hline 49. PNC_VST_NAPHSI.S & 50. SEX \\
\hline 51. SGA_10 & 52.SGA_2SD \\
\hline 53. YY4_LMP & 54. YY4_PCV \\
\hline 55. YY_LMP & 56. HISPANIC \\
\hline 57. URB_RUR & 58. DRK63B_A \\
\hline
\end{tabular}




\begin{tabular}{|l|l|}
\hline 59. DRK63L_A & 60. DRK6C_PG \\
\hline 61. INCOME5 & 62. MOM_BMI \\
\hline 63. MOM_BMIG & 64. MOM_HT_I \\
\hline 65. MOM_WT & 66. POB \\
\hline 67. PNCNO & 68. PNC_1TRM \\
\hline 69. PNC_WKS & 70. PP_NUMB \\
\hline 71. SMK6C_PG & 72. SMK6C_PP \\
\hline 73. STRS_TT3 & 74. STRS_T_G \\
\hline 75. SURE_WKS & 76. OUTCOME \\
\hline
\end{tabular}

\section{Appendix C- Description of ANN Final Network Parameters}

$\underline{\text { BORN Parous Method }}$

\begin{tabular}{|l|}
\hline FANN_FLO_2.1 \\
\hline num_layers $=3$ \\
\hline learning_rate $=0.100000$ \\
\hline connection_rate $=0.100000$ \\
\hline network_type $=0$ \\
\hline learning_momentum $=0.000000$ \\
\hline training_algorithm $=3$ \\
\hline train_error_function $=0$ \\
\hline
\end{tabular}




\begin{tabular}{|l}
\hline train_stop_function $=0$ \\
\hline cascade_output_change_fraction $=0.010000$ \\
\hline quickprop_decay $=-0.000100$ \\
\hline quickprop_mu=1.750000 \\
\hline rprop_increase_factor $=1.200000$ \\
\hline rprop_decrease_factor $=0.500000$ \\
\hline rprop_delta_min $=0.000000$ \\
\hline rprop_delta_max $=50.000000$ \\
\hline rprop_delta_zero $=0.100000$ \\
\hline cascade_output_stagnation_epochs $=12$ \\
\hline cascade_candidate_change_fraction $=0.010000$ \\
\hline cascade_candidate_stagnation_epochs $=12$ \\
\hline cascade_max_out_epochs $=150$ \\
\hline cascade_min_out_epochs $=50$ \\
\hline cascade_max_cand_epochs $=150$ \\
\hline cascade_min_cand_epochs $=50$ \\
\hline cascade_num_candidate_groups $=2$ \\
\hline cit_fail_limit $=3.49999994039535520000 \mathrm{e}-001$ \\
\hline cascade_candidate_limit $=1.00000000000000000000 \mathrm{e}+003$ \\
\hline
\end{tabular}


cascade_activation_functions=3 578101114151617

cascade_activation_steepnesses_count $=4$

cascade_activation_steepnesses $=2.5000000000000000000 \mathrm{e}-001$

5.00000000000000000000e-001 7.50000000000000000000e-001

$1.00000000000000000000 \mathrm{e}+000$

layer_sizes $=2422$

scale_included $=0$

neurons (num_inputs, activation_function, activation_steepness $)=(0,0$,

$0.00000000000000000000 \mathrm{e}+000)(0,0,0.00000000000000000000 \mathrm{e}+000)(0,0$,

$0.00000000000000000000 \mathrm{e}+000)(0,0,0.00000000000000000000 \mathrm{e}+000)(0,0$,

$0.00000000000000000000 \mathrm{e}+000)(0,0,0.00000000000000000000 \mathrm{e}+000)(0,0$,

$0.00000000000000000000 \mathrm{e}+000)(0,0,0.00000000000000000000 \mathrm{e}+000)(0,0$,

$0.00000000000000000000 \mathrm{e}+000)(0,0,0.00000000000000000000 \mathrm{e}+000)(0,0$,

$0.00000000000000000000 \mathrm{e}+000)(0,0,0.00000000000000000000 \mathrm{e}+000)(0,0$,

$0.00000000000000000000 \mathrm{e}+000)(0,0,0.0000000000000000000 \mathrm{e}+000)(0,0$,

$0.00000000000000000000 \mathrm{e}+000)(0,0,0.00000000000000000000 \mathrm{e}+000)(0,0$,

$0.00000000000000000000 \mathrm{e}+000)(0,0,0.00000000000000000000 \mathrm{e}+000)(0,0$,

$0.00000000000000000000 \mathrm{e}+000)(0,0,0.0000000000000000000 \mathrm{e}+000)(0,0$,

$0.0000000000000000000 \mathrm{e}+000)(0,0,0.00000000000000000000 \mathrm{e}+000)(0,0$,

$0.00000000000000000000 \mathrm{e}+000)(0,0,0.00000000000000000000 \mathrm{e}+000)(24,5$, 


\begin{tabular}{|l}
$5.00000000000000000000 \mathrm{e}-001)(0,5,5.00000000000000000000 \mathrm{e}-001)(2,5$, \\
$5.00000000000000000000 \mathrm{e}-001)(0,5,5.00000000000000000000 \mathrm{e}-001)$ \\
connections (connected_to_neuron, weight $)=(23,1.25688269734382630000 \mathrm{e}-001)(0$, \\
$2.66311973333358760000 \mathrm{e}-001)(1,6.09781086444854740000 \mathrm{e}-001)(2$, \\
$1.43165718764066700000 \mathrm{e}-002)(3,4.80457663536071780000 \mathrm{e}-001)(4$, \\
$1.53704524040222170000 \mathrm{e}-001)(5,3.56099337339401250000 \mathrm{e}-001)(6,-$ \\
$3.43392416834831240000 \mathrm{e}-002)(7,-1.38434067368507390000 \mathrm{e}-001)(8$, \\
$1.94338448345661160000 \mathrm{e}-002)(9,8.59185308218002320000 \mathrm{e}-002)(10$, \\
$1.06069691479206090000 \mathrm{e}-001)(11,6.98032155632972720000 \mathrm{e}-002)(12$, \\
$8.48777741193771360000 \mathrm{e}-002)(13,3.99983779061585660000 \mathrm{e}-004)(14$, \\
$6.62460774183273320000 \mathrm{e}-002)(15,-1.86994001269340520000 \mathrm{e}-002)(16$, \\
$4.35047894716262820000 \mathrm{e}-002)(17,8.23962539434432980000 \mathrm{e}-002)(18,-$ \\
$3.70812639594078060000 \mathrm{e}-002)(19,3.71759310364723210000 \mathrm{e}-002)(20$, \\
$3.57275269925594330000 \mathrm{e}-002)(21,5.73887117207050320000 \mathrm{e}-002)(22,-$ \\
$2.52741612493991850000 \mathrm{e}-002)(25,-7.18599511310458180000 \mathrm{e}-003)(24,-$ \\
$1.68719558715820310000 \mathrm{e}+001)$
\end{tabular}

$\underline{\text { BORN Nulliparous Method }}$

\section{FANN_FLO_2.1}

num_layers $=3$ 


\begin{tabular}{|l|}
\hline learning_rate $=0.900000$ \\
\hline connection_rate $=0.900000$ \\
\hline network_type $=0$ \\
\hline learning_momentum $=0.000000$ \\
\hline training_algorithm $=3$ \\
\hline train_error_function $=0$ \\
\hline train_stop_function $=0$ \\
\hline cascade_output_change_fraction $=0.010000$ \\
\hline quickprop_decay $=-0.000100$ \\
\hline quickprop_mu $=1.750000$ \\
\hline rprop_increase_factor $=1.200000$ \\
\hline rprop_decrease_factor $=0.500000$ \\
\hline rprop_delta_min $=0.000000$ \\
\hline rprop_delta_max $=50.000000$ \\
\hline rprop_delta_zero $=0.100000$ \\
\hline cascade_output_stagnation_epochs $=12$ \\
\hline cascade_candidate_change_fraction $=0.010000$ \\
\hline cascade_candidate_stagnation_epochs $=12$ \\
\hline cascade_max_out_epochs $=150$ \\
\hline cascade_min_out_epochs $=50$ \\
\hline cascade_max_cand_epochs $=150$ \\
\hline
\end{tabular}




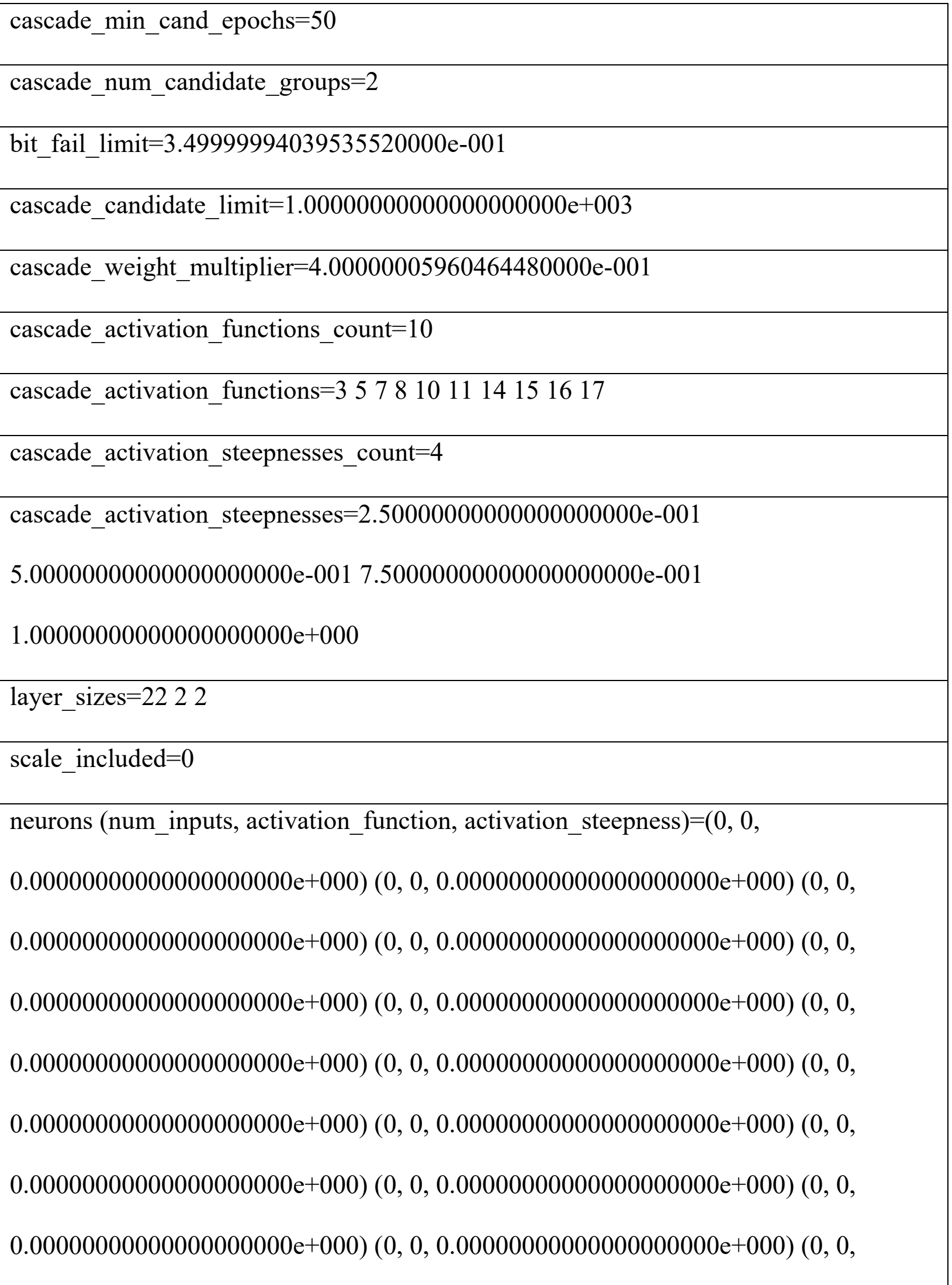




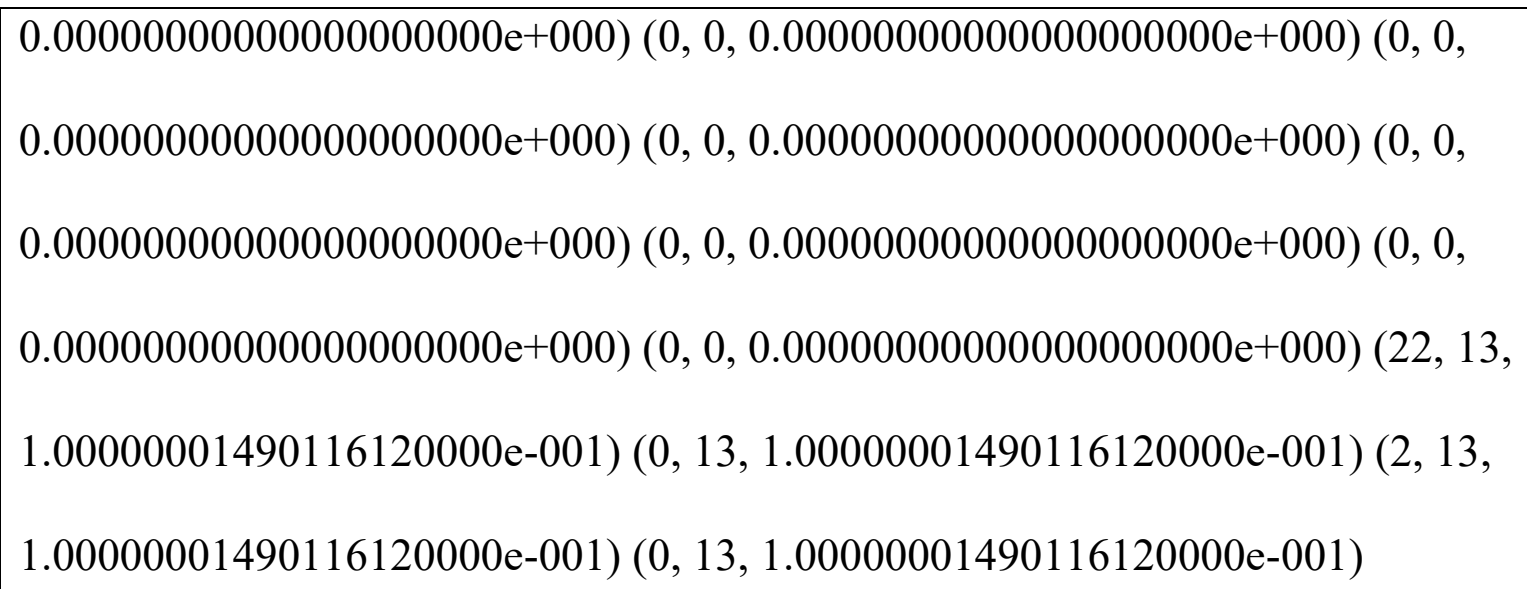




\section{PRAMS Parous Method}

\begin{tabular}{|l|}
\hline FANN_FLO_2.1 \\
\hline num_layers $=3$ \\
\hline learning_rate $=0.100000$ \\
\hline connection_rate $=0.900000$ \\
\hline network_type $=0$ \\
\hline learning_momentum $=0.000000$ \\
\hline training_algorithm $=3$ \\
\hline train_error_function $=0$ \\
\hline train_stop_function $=0$ \\
\hline cascade_output_change_fraction $=0.010000$ \\
\hline quickprop_decay $=-0.000100$ \\
\hline quickprop_mu $=1.750000$ \\
\hline rprop_increase_factor $=1.200000$ \\
\hline rprop_decrease_factor $=0.500000$ \\
\hline rprop_delta_min $=0.000000$ \\
\hline rprop_delta_max $=50.000000$ \\
\hline rprop_delta_zero $=0.100000$ \\
\hline cascade_output_stagnation_epochs $=12$ \\
\hline cascade_candidate_change_fraction $=0.010000$ \\
\hline
\end{tabular}




\begin{tabular}{|c|}
\hline cascade_max_out_epochs $=150$ \\
\hline cascade_min_out_epochs $=50$ \\
\hline cascade_max_cand_epochs $=150$ \\
\hline cascade_min_cand_epochs $=50$ \\
\hline cascade_num_candidate_groups $=2$ \\
\hline bit_fail_limit=3.49999994039535520000e-001 \\
\hline cascade_candidate_limit $=1.00000000000000000000 \mathrm{e}+003$ \\
\hline cascade_weight_multiplier=4.00000005960464480000e-001 \\
\hline cascade_activation_functions_count $=10$ \\
\hline cascade_activation_functions=35781011 \\
\hline cascade_activation_steepnesses_count $=4$ \\
\hline cascade_activation_steepnesses $=2.50000000000000000000 \mathrm{e}-001$ \\
\hline $5.00000000000000000000 \mathrm{e}-0017.50000000000000000000 \mathrm{e}-001$ \\
\hline $1.00000000000000000000 \mathrm{e}+000$ \\
\hline layer_sizes $=2222$ \\
\hline scale_included $=0$ \\
\hline neurons (num_inputs, activation_function, activation_steepness) $=(0,0$, \\
\hline $0.00000000000000000000 \mathrm{e}+000)(0,0,0.00000000000000000000 \mathrm{e}+000)(0,0$, \\
\hline $0.00000000000000000000 \mathrm{e}+000)(0,0,0.00000000000000000000 \mathrm{e}+000)(0,0$, \\
\hline $0.00000000000000000000 \mathrm{e}+000)(0,0,0.00000000000000000000 \mathrm{e}+000)(0,0$, \\
\hline
\end{tabular}




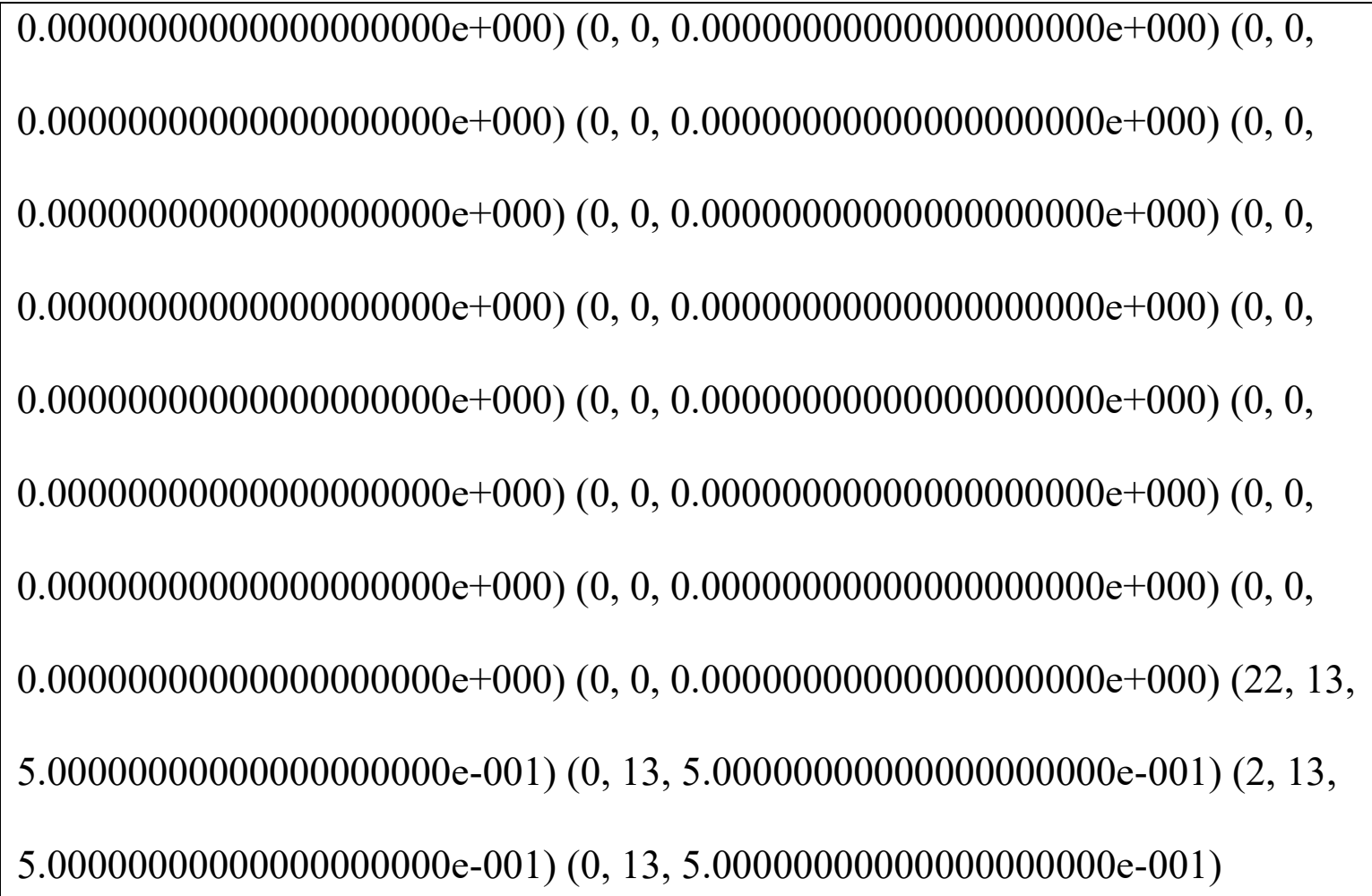




\section{$\underline{\text { PRAMS Nulliparous Method }}$}

\begin{tabular}{|l|}
\hline FANN_FLO_2.1 \\
\hline num_layers $=3$ \\
\hline learning_rate $=0.100000$ \\
\hline connection_rate $=0.100000$ \\
\hline network_type $=0$ \\
\hline learning_momentum $=0.000000$ \\
\hline training_algorithm $=3$ \\
\hline train_error_function $=0$ \\
\hline train_stop_function $=0$ \\
\hline cascade_output_change_fraction $=0.010000$ \\
\hline quickprop_decay $=-0.000100$ \\
\hline quickprop_mu $=1.750000$ \\
\hline rprop_increase_factor $=1.200000$ \\
\hline rprop_decrease_factor $=0.500000$ \\
\hline rprop_delta_min $=0.000000$ \\
\hline
\end{tabular}




\begin{tabular}{|l|}
\hline rprop_delta_max $=50.000000$ \\
\hline rprop_delta_zero $=0.100000$ \\
\hline cascade_output_stagnation_epochs $=12$ \\
\hline cascade_candidate_change_fraction $=0.010000$ \\
\hline cascade_candidate_stagnation_epochs $=12$ \\
\hline cascade_max_out_epochs $=150$ \\
\hline cascade_min_out_epochs $=50$ \\
\hline cascade_max_cand_epochs $=150$ \\
\hline cascade_min_cand_epochs $=50$ \\
\hline cascade_num_candidate_groups $=2$ \\
\hline bit_fail_limit $=3.49999994039535520000 \mathrm{e}-001$ \\
\hline cascade_candidate_limit $=1.00000000000000000000 \mathrm{e}+003$ \\
\hline cascade_weight_multiplier $=4.00000005960464480000 \mathrm{e}-001$ \\
\hline cascade_activation_functions_count $=10$ \\
\hline cascade_activation_functions $=35781011141617$ \\
\hline cascade_activation_steepnesses_count $=4$ \\
\hline cascade_activation_steepnesses $=2.50000000000000000000 \mathrm{e}-001$ \\
\hline scale_included $=0$ \\
\hline layer_sizes $=2022$ \\
\hline $00000000000000000000 \mathrm{e}-0017.50000000000000000000 \mathrm{e}-001$ \\
\hline
\end{tabular}




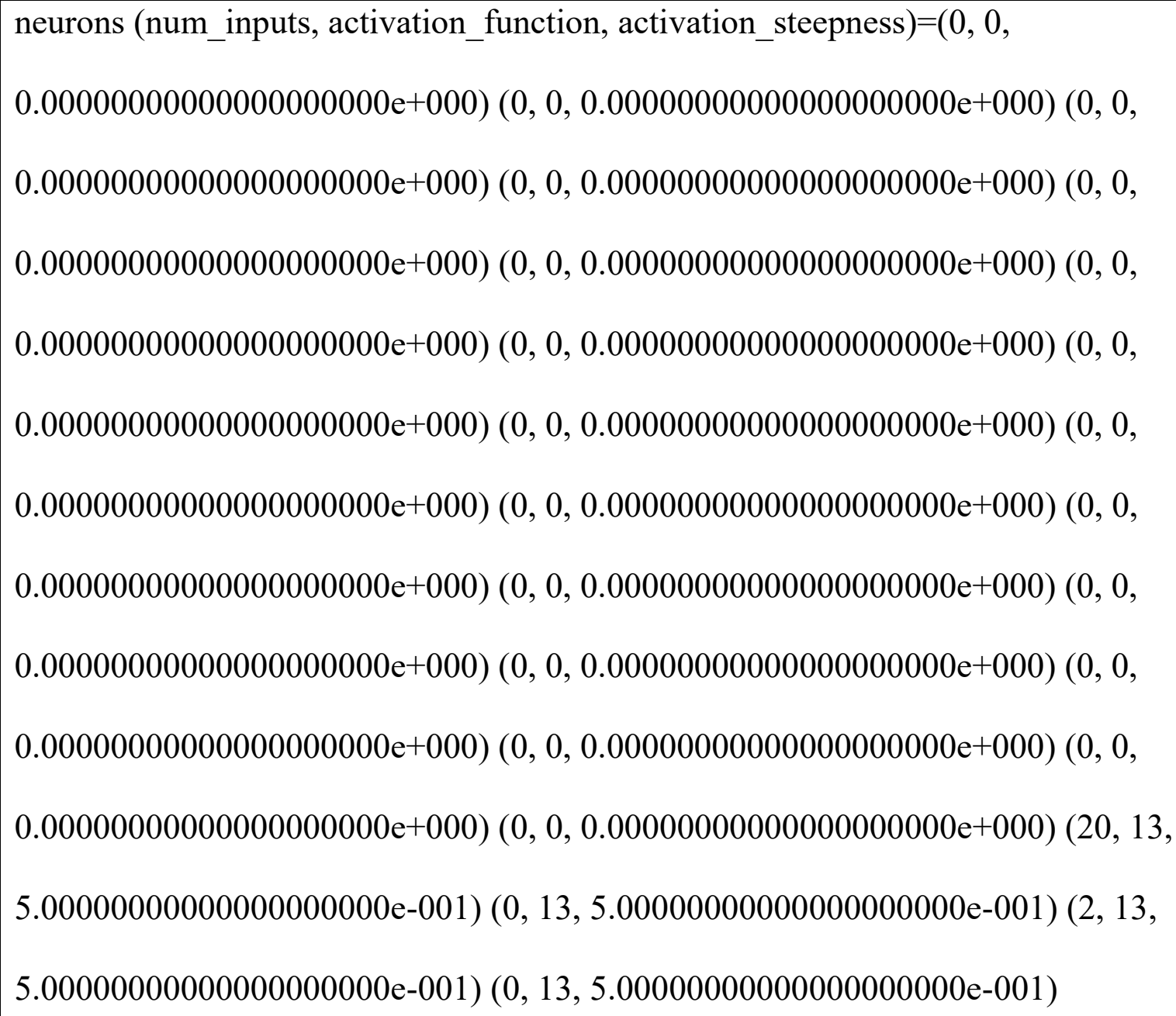




$$
\begin{aligned}
& 7.23048374056816100000 \mathrm{e}-002)(15,-7.50005841255187990000 \mathrm{e}-002)(16, \\
& 4.46150936186313630000 \mathrm{e}-002)(17,8.22790618985891340000 \mathrm{e}-003)(18,- \\
& 2.57767923176288600000 \mathrm{e}-002)(21,-3.702209517359733600000 \mathrm{e}-002)(20, \\
& 8.20150947570800780000 \mathrm{e}+000)
\end{aligned}
$$

